\title{
Band 1 Kapitel 5: Zusammenschau, Schlussfolgerungen und Perspektiven
}

\section{Volume 1 Chapter 5: Synthesis, Conclusions and Perspectives}

\author{
Koordinierende LeitautorInnen \\ Wolfgang Schöner, Andreas Gobiet, Helga Kromp-Kolb \\ LeitautorInnen \\ Reinhard Böhm ${ }^{\dagger}$, Michael Hofstätter, Maja Zuvela-Aloise \\ Beiträge von \\ Naumann Awan, Klaus Haslinger, Lukas Meyer, Peter Weish \\ Für den Begutachtungsprozess \\ Fred Luks
}

Inhalt

ZUSAMMENFASSUNG

SUMMARY

KERNAUSSAGEN

5.1 Motivation

5.2 Naturwissenschaft als empirische Wissenschaft mit ethischer Verantwortung

348

348

350

5.3 Zusammenschau: Klimaänderung in Österreich 1800-2100

5.4 Fallbeispiele: Vergangenes und künftiges Klima in Österreich

5.4.1 Klimaänderung im Gebirge - Ursachen und Wirkungen am Beispiel des Sonnblicks

5.4.2 Klimaänderung in der Stadt Wien

5.4.3 Klimaänderung und Seen - Der Neusiedlersee

5.4.4 Die Bedeutung des Mittelmeeres für das Klima in Österreich

5.4.5 Veränderung großräumiger Zirkulationsmuster („Wetterlagen") im Klimawandel in Mitteleuropa und ihre Bedeutung für Österreich

5.5 Die Entwicklung weiter denken: Überraschungen, abrupte Änderungen und KippPunkte im Klimasystem
354

354

357

362

364
5.6 Forschungsbedarf

5.7 Aussagen zum Klimawandel und deren Belastbarkeit

5.8 Literaturverzeichnis

374 


\section{ZUSAMMENFASSUNG}

In den letzten Jahrzehnten ist ein schlüssiges Bild des globalen Klimawandels, seiner Ursachen und der spezifischen Ausprägungen in Österreich entstanden. Das Bild ist jedoch von unterschiedlicher Schärfe und Belastbarkeit. Die Temperaturänderung der letzten 250 Jahre in Österreich ist etwa halb so groß wie der Anstieg, der bis Ende des Jahrhunderts zu erwarten ist, legt man ein „business-as-usual“ Szenario zugrunde. Beim Niederschlag ist hingegen die erwartete Änderung klein gegenüber den Unsicherheiten und wird vor allem im Sommer und erst in der zweiten Hälfte dieses Jahrhunderts deutlich ausgeprägt sein.

Die außerordentlich wertvolle Klimareihe des Sonnblickobservatoriums, am Alpenhauptkamm in 3106 m Seehöhe gelegen, zeigt seit dem Ende des 19. Jahrhunderts über den Temperaturanstieg hinaus eine deutliche Zunahme der Sonnenscheindauer und der Globalstrahlung sowie eine deutliche Verschiebung von Schneefall zu Regen. Ein beträchtlicher Rückgang der Gletscher und Auftauen des Permafrostes sind die Folgen.

Wie in allen größeren Städten überlagern sich in Wien städtische Wärmeinsel und klimawandelbedingter Temperaturanstieg und bedingen besonders hohe Temperaturen in dicht verbauten Gebieten. Charakteristisch ist die geringer werdende nächtliche Abkühlung. Im Laufe dieses Jahrhunderts ergibt sich, unabhängig vom zugrunde gelegten Szenario, beträchtlicher Anpassungsbedarf in stadtplanerischer Hinsicht und bei Gebäuden. Weiterer Zuzug und die erwartete Verdichtung und Ausweitung des verbauten Gebietes werden die Situation verschärfen.

Für das Hochwasserrisiko in Österreich ist das Klima im Mittelmeerraum von besonderer Bedeutung, entstehen doch über dem Golf von Genua jene Tiefdruckgebiete, die als „VbLagen“ im Alpenraum zu beträchtlichen Niederschlägen führen können. Wiewohl es noch nicht möglich ist künftige Änderungen in der Häufigkeit der Vb-Lagen zu quantifizieren, ist doch deutlich, dass ein wärmeres Mittelmeer zu niederschlagsreicheren $\mathrm{Vb}$-Lagen führen kann.

Für viele gesellschaftliche Fragestellungen genügt der Stand des Wissens über Klimawandel in Österreich; er stellt die Notwendigkeit von Minderungs- und Anpassungsmaßnahmen außer Frage. Noch offene Fragen und Unsicherheiten, ebenso wie die Scheu vor den sich ergebenden gesellschaftlichen Konsequenzen, lassen jedoch die Debatte um die Notwendigkeit und Dringlichkeit von Klimaschutz und Anpassung an den Klimawandel nicht verstummen. Als empirische Wissenschaft kann die Klimatologie grundsätzlich keine strengen Beweise für ihre Theorie des anthropogenen Klimawandels liefern, doch ist deren Kern über zahlreiche Beobachtungen, theoretische Überlegungen und Modellberechnungen gut abgesichert.

Davon getrennt sind umweltethische und gesellschaftspolitische Aspekte zu beachten. Da nicht alle möglichen Konsequenzen des Klimawandels aus ethischer Sicht gleichermaßen wichtig sind, ist es im Sinne einer vorausschauenden Risikobetrachtung notwendig, die obere Grenze möglicher negativer Umweltauswirkungen (das „worst case“ Szenario) anzunehmen. Dies entspricht dem Sicherheitsprinzip. Ziel des daran anschließenden Vorsorgeprinzips ist es, das Auftreten irreparabler Schäden, etwa der Gesundheit oder von Ökosystemen, durch geeignete Maßnahmen von vornherein auszuschließen. Daraus folgt, dass die volle Bandbreite möglicher Auswirkungen des Klimawandels darzustellen ist, einschließlich „best case" und „worst case" Szenarien, um der Gesellschaft informierte Entscheidungen zu ermöglichen. In diesem Sinn müssen auch abrupte Änderungen und Kipp-Punkte des Klimasystems als Möglichkeit in Betracht gezogen werden.

\section{SUMMARY}

Over the past few decades, a coherent picture of global climate change and its causes and impacts on Austria has been developed. Yet this is a picture of varying accuracy and robustness. During the past 250 years, the observed temperature increase in Austria equals approximately half of that which is expected to occur by 2100 , assuming a „business-as-usual“ scenario. The expected changes in precipitation, while small compared to their associated uncertainties, will only become more pronounced in the second half of this century and particularly in summertime.

The unique observational dataset of the Sonnblick Observatory (3106 m above sea level on the main crest of the Alps) indicates not only rising temperatures since the end of the $19^{\text {th }}$ century, but also marked increases in sunshine duration and global radiation, as well as a clear shift from solid (snow) to liquid precipitation (rain). Consequently, glaciers are shrinking considerably and permafrost is thawing.

Like in all larger cities, the temperature increase in Vienna is a combined effect of both global warming and the urban heat island effect. In recent years, particularly high temperatures were observed in built-up areas as well as a characteristic reduction in nighttime cooling. Within the $21^{\text {st }}$ century, regardless of the emission scenarios assumed, a considerable need for climate-change adaptation with regard to spatial planning and buildings will arise. In addition, growing populations and increased density and the extension of built-up areas will create mounting pressure. 
It is possible that Lake Neusiedl, being a steppe lake, will dry-up as a result of climate variations, as known from historical events. Due to increased warming, evaporation from this shallow lake is projected to increase to an extent that cannot be compensated by increased precipitation, which itself still remains subject to uncertainty. The possibility exists that precipitation will decrease, further enhancing the risk of the lake drying.

The Mediterranean Sea is an important factor with regard to flood risks in Austria, as low pressure systems originating over the gulf of Genoa, particularly Vb cyclones, can cause considerable precipitation in the Alpine area. Although it is currently not possible to quantify future changes in the frequency of such cyclones, a warming of the Mediterranean Sea could lead to $\mathrm{Vb}$ cyclones associated with more intense precipitation.

During the past century, the frequency of different circulation patterns has changed: While the influence of meridional (south-north oriented) circulation patterns has increased, the influence of zonal (west-east oriented) patterns associated with air masses from the Atlantic Ocean has decreased over Central Europe. Although climate models have known weaknesses in correctly representing the statistical properties of circulation patterns, there are indications that high-pressure situations may become more frequent over Western and South-Western Europe in the future.

The state of climate change knowledge in Austria clearly indicates the need for mitigation and adaptation measures. However, open questions and uncertainties which remain, as well as hesitation with regard to the necessary societal changes, have led the discussion about the need and urgency for climate protection and adaptation to climate change to be continued. As an empirical science, climate science cannot deliver strict proof for its climate theory. However, the core results are very well backed-up by observations, theoretical considerations, and model simulations.

\section{KERNAUSSAGEN}

- In den letzten Jahrzehnten ist ein schlüssiges Bild des globalen Klimawandels, seiner Ursachen und der spezifischen Ausprägungen in Österreich entstanden.

Das Bild ist jedoch von unterschiedlicher Schärfe und Belastbarkeit: mittlere Veränderungen sind besser belegt und besser abschätzbar als Extremereignisse, großräumigere Aussagen belastbarer als lokale, Aussagen über Veränderungen der Temperatur wesentlich robuster als solche über den Niederschlag und kleinräumige Gewitter schwerer erfassbar als mit großräumigen Fronten verbundene Niederschläge.
- Die in den letzten 250 Jahren beobachteten Änderungen sind gering im Vergleich zu den bis Ende 2100 zu erwartenden.

Dies gilt unabhängig vom Szenario, das zugrundegelegt wird (sehr wahrscheinlich). In der zweiten Hälfte des Jahrhunderts werden die Änderungen wahrscheinlich rascher erfolgen als in der ersten. Aufgrund der großen natürlichen Variabilität der Niederschlagssummen im Sommer, sind in den Klimaprojektionen Änderungen dieser Klimagröße dann erst signifikant und daher nachweisbar.

- Klimaänderungen zeigen ein konsistentes Bild in typischen geographischen Gegebenheiten Österreichs

Die Klimareihen des Hohen Sonnblicks, als Beispiel für den Gebirgsraum, zeigen über den Temperaturanstieg hinaus eine deutliche Zunahme der Sonnenscheindauer und der Globalstrahlung sowie eine deutliche Verschiebung von Schneefall zu Regen. Beträchtlicher Rückgang der Gletscher und Auftauen des Permafrostes sind für den Hohen Sonnblick ebenfalls dokumentiert.

- In Wien, als Beispiel für den urbanen Raum, überlagern sich städtische Wärmeinsel und klimawandelbedingter Temperaturanstieg.

Besonders hohe Temperaturen in dicht verbauten Gebieten, und abnehmende nächtliche Abkühlung sind die Folge. Diese Entwicklung wird sich in den kommenden Jahrzehnten fortsetzen (sehr wahrscheinlich).

- $\quad$ Für das Hochwasserrisiko in Österreich ist das Klima im Mittelmeerraum von besonderer Bedeutung.

Wiewohl es noch nicht möglich ist künftige Änderungen der Häufigkeit der niederschlagsreichen „Vb-Lagen“ zu quantifizieren, ist doch wahrscheinlich, dass ein wärmeres Mittelmeer zu niederschlagsreicheren $\mathrm{Vb}$-Lagen führen wird.

- Die Klimawissenschaft muss die volle Bandbreite möglicher Auswirkungen darstellen.

"Best case" und "worst case" Szenarien müssen bekannt sein, um der Gesellschaft informierte Entscheidungen zu ermöglichen. In diesem Sinn müssen auch abrupte Änderungen und Kipp-Punkte des Klimasystems als Möglichkeit in Betracht gezogen werden. 


\subsection{Motivation \\ 5.1 Motivation}

Die vorangegangenen Kapitel haben anhand von Daten und Fakten aufgezeigt, wie sich das Klima in Österreich in der Vergangenheit verändert hat und gegenwärtig verändert. Aus Modellsimulationen wurden, aufbauend auf Szenarien des globalen Klimawandels, mögliche Entwicklungen des Klimas für die Zukunft aufgezeigt. Trotz aller Fehlerquellen und Unsicherheiten, auf die explizit hingewiesen wurde, ergibt sich ein schlüssiges Bild des Klimawandels und seiner Ursachen. Das Bild ist jedoch von unterschiedlicher Schärfe und Belastbarkeit. Für viele gesellschaftliche Fragestellungen ist der jeweils verfügbare Wissensstand ausreichend. Dazu gehört z. B. die grundsätzliche Frage nach der Notwendigkeit von Minderungs- und Anpassungsmaßnahmen, der in Band 3 nachgegangen wird. Auch viele Auswirkungen künftiger Klimaänderungen lassen sich anhand des gegenwärtigen Verständnisses um den Klimawandel beantworten. Oft lassen sich Bandbreiten ableiten, die hinreichend Information als Grundlage für Entscheidungen liefern. Beispiele dafür finden sich in den beiden Folgebänden, vor allem aber in Band 2, der sich den Auswirkungen des Klimawandels auf natürliche und gesellschaftlich-wirtschaftliche Systeme widmet.

In diesem abschließenden Kapitel des ersten Bandes sollen die Aussagen der vorhergehenden Kapitel zu einer Gesamtsicht zusammengeführt und daraus Schlussfolgerungen abgeleitet werden. Um die Anschaulichkeit der Aussagen über den Klimawandel in Österreich zu erhöhen, werden am Beispiel von ausgewählten Fallstudien diese Veränderungen nochmals übersichtlich und im Sinne eines abgerundeten Bildes dargestellt. Die Fallstudien beschreiben einerseits den Klimawandel in für Österreich typischen geographischen Gegebenheiten (Gebirge, Stadt, See), andererseits relevante Einflussgrößen auf das Klima Österreichs (Mittelmeer, Wetterlagen), die noch eines vertieften Verständnisses und weiterer Forschung in Zukunft bedürfen.

Darüber hinaus - und auch dafür kann die Vergangenheit als Beispiel dienen - ist in Zukunft auch mit Überraschungen zu rechnen: Entwicklungen, die, wenn sie einsetzen, oft recht gut erklärt werden können, allerdings vorher nicht bedacht wurden, aber auch Entwicklungen, die nicht unmittelbar interpretierbar sind und die helfen, das Verständnis des Klimasystems voranzutreiben. Lücken im Systemverständnis und Überraschungen im Zuge wissenschaftlicher Arbeit können ein wesentlicher Motor für den Fortschritt und die Wissensgenerierung sein. Das vorliegende Kapitel gibt hierfür Beispiele.

Ein eigener Abschnitt wird dem Forschungsbedarf gewidmet. Auch hier geht es um eine Gesamtschau unter Berück- sichtigung von Überschneidungen und Synergien. Schließlich werden im letzten Abschnitt Kernaussagen zum Klimawandel aus dem Bereich der naturwissenschaftlichen Grundlagen angeführt, die in der Öffentlichkeit oft kontrovers diskutiert werden, wobei eine Bewertung hinsichtlich des Grades der Sicherheit (welcher z. B. aus Qualität und Umfang der Datenbasis abgeschätzt wird) einerseits und des Grads der Übereinstimmung unter WissenschaftlerInnen andererseits, als Hilfe zur Einordnung dieses Wissens verwendet wird. Auch für diesen Abschnitt ist das Zusammenführen von Kernaussagen der Einzelkapitel zu einer Gesamtsicht eine wesentliche Motivation. So gewinnen etwa manche Aussagen über zukünftige Veränderungen des Klimas erst auf Basis des Vergleichs mit den Veränderungen in der Vergangenheit an Schärfe.

All diesen Ausführungen vorangestellt sind Überlegungen zum wissenschaftlichen Erkenntnisprozesses und der Ethik in der Wissenschaft. Klimaforschung, als empirische Wissenschaft, kann keine strengen Beweise liefern, die der Diskussion um den Klimawandel ein klares Ende setzen könnten. Als politisch relevante Wissenschaft steht die Klimaforschung sehr oft unter mehrfachem Druck: Resultate werden oft rascher gebraucht als sie gut abgesichert verfügbar sind und sie fordern Konsequenzen, die einschneidend sein können. Dass derartige Randbedingungen sich auch auf die Qualität der Ergebnisse der Klimaforschung auswirken können, insbesondere aber auf deren Kommunikation, ist naheliegend.

\subsection{Naturwissenschaft als empirische Wissenschaft mit ethischer Verantwortung}

\subsection{Natural sciences as empirical science with ethical responsibility}

„Die Klimaforschung steht als politisch relevante Wissenschaft unter dem Druck, schnell Resultate zu liefern. Und wo diese Resultate kontrovers sind, entsteht in der Öffentlichkeit rasch der Eindruck mangelnder Glaubwürdigkeit. Forschung wird genau dann zum Gegenstand öffentlicher Glaubwürdigkeitsdebatten, wenn epistemische Unsicherheiten auf politische, moralische, soziale oder ökonomische Interessen treffen" (Leuschner, 2012).

In der Öffentlichkeit und auch unter WissenschaftlerInnen, deren Expertise nicht im Bereich der Klimatologie oder der Meteorologie liegt, herrscht manchmal Unsicherheit hinsichtlich der wissenschaftlichen Zuverlässigkeit der den anthropogenen Klimawandel betreffenden Feststellungen. Das ist nicht verwunderlich, da Meldungen in den Medien und populärwissenschaftliche Bücher und Filme ein breites Spekt- 
rum von Interpretationen anbieten, von der Klimaapokalypse (siehe z. B. den Film „The day after tomorrow" von Roland Emmerich, 2004) über die Verschwörung geld- und machtgieriger WissenschafterInnen (siehe z.B. Crichton, 2004) bis zur rosigen Zukunft in einer wärmeren Welt (siehe z. B. Horx, 2007). Wertvolle Beiträge zur Erklärung, warum jenseits oberflächlicher Geplänkel eine einhellige Meinung zum Klimawandel nicht zu erwarten ist, liefern z. B. Hulme (2009) in einer umfassenden Analyse der kulturellen Dimension des Begriffes Klima, sowie Oreskes und Conway (2010) in einer stark USA-bezogenen Analyse politischer Ideologien als entscheidender Faktor in der Diskussion.

Die Klimafrage ist kein rein akademisches Problem, daher müssen in der Diskussion empirisch wissenschaftliche und erkenntnistheoretische Fragen sauber von umweltethischen und gesellschaftspolitischen Argumenten getrennt werden. Erkenntnistheoretisch ist ein strenger Beweis für den Klimawandel und dessen Ursachen nicht möglich, weil sich die Klimaforschung als eine empirische Wissenschaft versteht, die auf Beobachtung und Erfahrung gründet. Naturwissenschaftliche Theorien sind durch Beobachtungen geprüfte Hypothesen, die durch weitere Beobachtungen entweder erhärtet oder widerlegt werden (siehe z. B. Feynman, 1955). Nach Popper und dem Wiener Positivismus ist es sogar die vornehmliche Aufgabe der Wissenschaft, zu versuchen, die Hypothesen bzw. Theorien zu widerlegen, um so ihre Robustheit und Erklärungsmacht zu stärken (siehe z. B. Popper, 1955). Im praktischen Leben werden viele naturwissenschaftliche Hypothesen und Theorien, wie etwa die Existenz der Schwerkraft, erfolgreich als bewiesenes „Wissen“ gehandhabt, ja die gesamte technologische Entwicklung des letzten Jahrhunderts basiert auf solchen Theorien.

Im Lichte dessen kann im Zusammenhang mit dem Klimawandel zwar festgehalten werden, was beobachtet wurde, Deutungen, kausale Erklärungsversuche und Verallgemeinerung können aber aus erkenntnistheoretischer Sicht nie „bewiesen“ werden. Dass der Klimawandel etwa anthropogen verursacht ist, kann daher streng genommen nie bewiesen werden. Die Theorie des vom Menschen verursachten Klimawandels ist jedoch durch Modellexperimente belegt und sie ist anderen Theorien oder Hypothesen in Bezug auf Theorieüberprüfungen (z. B. Falsifizierungsversuchen) überlegen. Da sie darüber hinaus bereits seit über 20 Jahren der wissenschaftlichen Überprüfung unterworfen war, ist sie als wesentlich wahrscheinlicher einzuschätzen als andere Hypothesen und es ist sachlich gerechtfertigt, sie weiteren Betrachtungen und auch Entscheidungen zugrunde zu legen. Diesen Überlegungen zufolge ist es jedoch wichtig, Aussagen über den Klimawandel und seine
Folgeerscheinungen durch nachvollziehbare Bewertungen der Unsicherheiten zu ergänzen.

Hinsichtlich des umweltethischen und gesellschaftspolitischen Aspektes ist festzuhalten, dass man früher vielleicht noch sagen konnte, das Privileg der WissenschaftlerInnen bestünde darin, sich folgenlos irren zu dürfen. Heute können wissenschaftlich-technische Entwicklungen die Welt in gefährlicher Weise bedrohen - die Verantwortung der WissenschaftlerInnen hat daher zugenommen. Im konkreten Fall des Klimawandels ist die Wissenschaft z. B. dazu aufgerufen zu klären, was unter „dangerous anthropogenic interference“ (gefährliche anthropogene Störung) im Klimasystem zu verstehen ist, da die Staaten sich in der Klimarahmenkonvention (UNFCCC, 1992, Artikel 2) verpflichtet haben diese zu vermeiden:

„Das Endziel dieses Übereinkommens und aller damit zusammenhängenden Rechtsinstrumente, welche die Konferenz der Vertragsparteien beschließt, ist es, in Übereinstimmung mit den einschlägigen Bestimmungen des Übereinkommens die Stabilisierung der Treibhausgaskonzentrationen in der Atmosphäre auf einem Niveau zu erreichen, auf dem eine gefährliche anthropogene Störung des Klimasystems verhindert wird. Ein solches Niveau sollte innerhalb eines Zeitraums erreicht werden, der ausreicht, damit sich die Ökosysteme auf natürliche Weise den Klimaänderungen anpassen können, die Nahrungsmittelerzeugung nicht bedroht wird und die wirtschaftliche Entwicklung auf nachhaltige Weise fortgeführt werden kann."

Von dieser Definition, bzw. den dahinter liegenden, meist naturwissenschaftlichen Untersuchungen, hängen u.a. Art und Ausmaß der Eingriffe in die Entwicklung des Energieund Wirtschaftssystems ab. Wie tiefgreifend diese sein können, lässt sich u.a. am Ringen um einen Paradigmenwechsel in den an Wachstum orientierten Wirtschaftstheorien erkennen (vgl. Daly, 1990). Wie eingangs beschrieben, sind naturwissenschaftliche und damit auch klimawissenschaftliche Erkenntnisse mit Unsicherheiten behaftet, sodass immer von mehreren Entwicklungsmöglichkeiten des Klimas und dementsprechend auch der jeweiligen Folgen ausgegangen werden muss. Nicht alle wahrscheinlichen oder möglichen Konsequenzen des Klimawandels sind aus ethischer Sicht gleichermaßen wichtig. Sollen Strategien des Umgangs mit dem Klimawandel bewertet werden, sind jedenfalls jene Konsequenzen zu betrachten, die das Risiko der Verletzung grundlegender Rechte von Menschen bergen. Weitgehend unumstritten ist, dass auch zukünftig lebende Menschen Anspruchsrechte haben, die heute lebende Menschen zu respektieren haben. Seit dem „Brundtland-Bericht“ (1987) ist „sustainable development“ („Dauerhafte [nachhaltige] Entwicklung ist Entwicklung, die die Bedürfnisse der Gegenwart befriedigt, ohne zu riskieren, 
dass künftige Generationen ihre eigenen Bedürfnisse nicht befriedigen können.") immer wieder auf internationaler Ebene als Handlungsprinzip - etwa in der Agenda 21 und in den Strategien für eine Nachhaltige Entwicklung der EU - bekräftigt worden. Auch die in Ausarbeitung befindlichen globalen Sustainable Development Goals, die 2015 verabschiedet werden sollen, fußen darauf. „Sustainable development “ ist daher als internationaler ethischer Grundkonsens aufzufassen.

Vielfach wird jedoch der anthropozentrische, ausschließlich auf das Wohlergehen des Menschen ausgerichtete Ansatz der Brundtland Definition als zu eng angesehen. So meint Spaemann (1979) dass der Mensch, solange er die Natur ausschließlich funktional auf seine Bedürfnisse hin interpretiert, im Zuge einer Güterabwägung jeweils von der Natur nur das übrig lassen wird, was bei einer solchen Abwägung im Augenblick noch ungeschoren davonkommt. Der Anteil der Natur werde dabei ständig verkürzt, sodass der Mensch letztlich seine eigene Existenzgrundlage zerstört.

Unabhängig von diesen grundlegenden Fragen des Weltbildes weisen die IPCC-Berichte jedenfalls aus, dass die meisten Szenarien des Klimawandels grundlegende Rechte zukünftig Lebender (z. B. auf Überleben, Gesundheit und Autonomie) verletzen, bei extremeren Szenarien tun sie dies sogar in hohem Maße. Grundlegende Rechte nicht zu verletzen und zu schützen ist eine minimale Forderung der Gerechtigkeit. Aus der Perspektive der Gerechtigkeit ist die Verletzung von Rechten sowohl heute als auch zukünftig Lebender zu vermeiden. Eine Klimapolitik, die viele Menschen vermeidbar dem Risiko der Verletzung ihrer grundlegenden Rechte aussetzt, ist demnach ethisch unzulässig, selbst wenn dieses Risiko gering sein sollte. Das entspricht der Forderung von Hans Jonas, dass einer einigermaßen gut begründeten Unheilsprognose Vorrang vor der Heilsprognose zu geben ist (Jonas, 1979). Jonas begründet damit philosophisch das Sicherheitsprinzip. Andererseits können auch Klimaschutzmaßnahmen, insbesondere wenn unreflektiert oder überhastet getroffen, Menschenleben kosten. Das System Erde ist ein komplexes, gekoppeltes System, das die globale Gesellschaft und Wirtschaft einschließt, die ihrerseits wieder stark gekoppelt und von einander abhängig sind (Planet under Pressure, 2012). Ethisches Handeln erfordert daher gesamtheitliche Betrachtungsweisen.

Zur Orientierung können drei Prinzipien der Umweltethik herangezogen werden, die sich in unterschiedlicher Form auch in den Rechtskörpern vieler Staaten wiederfinden: das Sicherheitsprinzip, das Vorsorgeprinzip und das Verursacherprinzip. Das Sicherheitsprinzip verlangt, im Zweifel für mögliche negative Umweltauswirkungen deren obere Grenze (das „worst case scenario") anzunehmen. Analog zum Grundsatz in $d u-$ bio pro reo (im Zweifel für den Angeklagten) der Rechtssprechung, soll der Grundsatz in dubio pro securitate (im Zweifel für die Sicherheit) gelten. Dies entspricht auch üblichem, vorausschauendem Umgang mit Risiko: Die Frage ob Klimaschutzmaßnahmen zu treffen sind oder nicht, lässt sich z. B. in einer Matrix mit vier Feldern abarbeiten, die aufgespannt werden von Zutreffen oder Nicht-Zutreffen der Theorie des anthropogenen Klimawandels auf der einen Achse und Klimaschutzmaßnahmen-Setzen oder Nicht-Setzen auf der anderen (Craven, 2009). Betrachtet man die jeweils ungünstigsten Konsequenzen in jedem der vier Felder, so zeigt sich, dass im Fall des Ergreifens von Maßnahmen im schlimmsten Fall die Wirtschaft ohne Notwendigkeit beeinträchtigt wird - aber selbst das ist umstritten - weil Klimaschutzmaßnahmen auch wirtschaftliche Vorteile bringen können. Die schlimmsten Konsequenzen wenn keine Maßnahmen getroffen werden sind hingegen klimabedingte Extremereignisse, Verlust von Land, Hungersnöte, etc. und, wenn Kipp-Punkte einbezogen werden, die Unmöglichkeit das Klima noch zu stabilisieren. Vom Standpunkt der Risikobetrachtung sind daher, unabhängig vom Zutreffen der Theorie des anthropogenen Klimawandels und ganz im Sinn des Sicherheitsprinzips, wenn auch nicht direkt daraus ableitbar, Klimaschutzmaßnahmen zu setzen.

Das Vorsorgeprinzip baut auf dem Sicherheitsprinzip auf und bezweckt, das Auftreten irreparabler Gesundheits- und Ökosystemschäden von vornherein auszuschließen, indem riskante Unternehmungen nicht zugelassen werden. Allerdings bleibt uns im Falle der Klimapolitik möglicherweise nur mehr die Wahl zwischen Optionen, die alle irreparablen Schäden als Konsequenz des Klimawandels beinhalten. Die Erklärung der UN-Konferenz für Umwelt und Entwicklung (UNCED) 1992 in Rio konkretisiert das Vorsorgeprinzip in Kapitel 35 Absatz 3 der Agenda 21:

„Angesichts der Gefahr irreversibler Umweltschäden soll ein Mangel an vollständiger wissenschaftlicher Gewissheit nicht als Entschuldigung dafür dienen, Maßnahmen hinauszuzögern, die in sich selbst gerechtfertigt sind. Bei Maßnahmen, die sich auf komplexe Systeme beziehen, die noch nicht voll verstanden worden sind und bei denen die Folgewirkungen von Störungen noch nicht vorausgesagt werden können, könnte der Vorsorgeansatz als Ausgangsbasis dienen."

Ein über jeden Zweifel erhabener wissenschaftlicher Nachweis negativer Folgen ist demnach zum Setzen von Maßnahmen nicht erforderlich, es genügt ein plausibel begründeter Verdacht. Daher sind Zweifel an der anthropogenen Beeinflussung des Klimas - selbst wenn sie aus rein akademischer Sicht gerechtfertigt wären - nicht als Rechtfertigung eines „business as usual“ zu akzeptieren, umso mehr, als die gleichen Maßnah- 
men, die zum „Klimaschutz" gefordert werden, auch andere positive Auswirkungen haben und z. B. für die notwendige Energiewende unabdingbar sind. Dass die Energiewende und der Übergang zu einer nachhaltigen Gesellschaft eine Vielzahl von bedeutenden Vorteilen bringen kann, wird im Global Energy Assessment (GEA, 2012) ausführlich dargestellt.

Das Verursacherprinzip sieht vor - im Sinne der Verantwortungsethik - jedeN VerursacherIn von Gesundheits- und Ökosystemschäden konsequent und im allgemeinsten Sinn zur Verantwortung zu ziehen, auch wenn der rechtliche Rahmen dafür vielfach noch fehlen mag. Die konkrete Umsetzung dieses Prinzips sorgt z. B. in den internationalen Klimakonferenzen (COP) für heftige und bisher ergebnislose Diskussionen über die Anrechnung bisheriger Treibhausgasemissionen (THG-Emissionen) und deren Bewertung in Hinblick auf künftige Minderungs- und Anpassungsmaßnahmen und deren Finanzierung.

In diesen Themenkomplex eingebettet lässt sich für die Klimawissenschaft ableiten, dass sie jedenfalls die volle Bandbreite möglicher Auswirkungen darstellen muss, einschließlich „best case“ und „worst case“ Szenarien, um der Gesellschaft informierte Entscheidungen zu ermöglichen.

\subsection{Zusammenschau: Klimaänderung in Österreich 1800-2100}

5.3 Synopsis: climate change in Austria 1800-2100

In den letzten Jahrzehnten ist ein schlüssiges Bild des globalen Klimawandels, seiner Ursachen und der spezifischen Ausprägungen in Österreich entstanden. Das Bild ist jedoch von unterschiedlicher Schärfe und Belastbarkeit: Mittlere Veränderungen sind besser belegt und besser abschätzbar als Extremereignisse, Aussagen über Veränderungen der Temperatur wesentlich robuster, als solche über den Niederschlag, großräumigere Aussagen belastbarer als lokale, daher z. B. kleinräumige Gewitter schwerer erfassbar als mit großräumigen Fronten verbundene Niederschläge.

Die Veränderungen der mittleren Verhältnisse des Klimas in Österreich hinsichtlich Temperatur und Niederschlag können für die Vergangenheit sehr gut durch den HISTALP Datensatz beschrieben werden (siehe Band 1, Kapitel 3). So ist etwa die Temperatur in Österreich seit 1880 um nahezu $2^{\circ} \mathrm{C}$ gestiegen, verglichen mit einer globalen Erhöhung um $0,85^{\circ} \mathrm{C}$ (siehe Band 1, Kapitel 1). Der erhöhte Anstieg ist speziell auch für die Zeit ab 1980 beobachtbar, in der dem globalen Anstieg von etwa $0,5^{\circ} \mathrm{C}$ eine Temperaturzunahme von etwa $1^{\circ} \mathrm{C}$ in
Österreich gegenüber steht. Wesentlich uneinheitlicher stellt sich die Niederschlagsentwicklung der letzten 150 Jahren dar: Sie zeigt deutliche regionale Unterschiede mit einer Zunahme in Westösterreich um etwa $+10 \%$ bis $+15 \%$ und einer Abnahme im Südosten in ähnlicher Größenordnung. Auch für andere meteorologische Elemente gibt es in Österreich gute Beobachtungen, so kann etwa gezeigt werden, dass in den letzten 130 Jahren insbesondere im Sommerhalbjahr die Sonnenscheindauer an den Bergstationen der Alpen deutlich zugenommen hat (siehe Band 1, Kapitel 3). Aus derartigen Änderungen lassen sich weitere Konsequenzen ableiten, die auch instrumentell bestätigt werden konnten, wie etwa eine Abnahme der Dauer der Schneebedeckung und ein Rückgang der Gletscher (siehe Fallbeispiel Abschnitt 5.4.1).

Für die Beschreibung von Projektionen des Klimas in die Zukunft stehen zum Zeitpunkt der Verfassung dieses Berichts als aktuellste Datenquellen die Simulationen aus dem EU-Projekt Ensembles sowie insbesondere aus dem nationalen Projekt reclip:century zur Verfügung (siehe Band 1, Kapitel 4). In den Abbildungen 5.1 und 5.2 sind die Entwicklungen der Vergangenheit seit 1800 mit den Szenarien für die Zukunft verknüpft. Basis für die zukünftige Entwicklung stellt ein Szenario mit sehr raschem Wirtschaftswachstum, Stabilisierung des Weltbevölkerung Mitte des Jahrhunderts, rascher Verbreitung neuer, effizienterer Technologien und ausgewogener Mischung von Energieträgern dar (SRES Szenario A1B).

Die Projektionen für die Temperatur sind robust und zeigen in der ersten Hälfte des 21. Jahrhunderts einen klaren Anstieg von etwa $1,4^{\circ} \mathrm{C}$. In der zweiten Hälfte des 21 . Jahrhunderts erhöht er sich für das gewählte Szenario auf $3,5^{\circ} \mathrm{C}$.

Bei den Niederschlagssummen ist weder in der Vergangenheit, noch in der Zukunft ein vergleichbar klarer Trend wie bei der Temperatur zu erkennen. Tendenziell ist im Winter eine Zunahme und im Sommer eine Abnahme der Niederschlagssummen zu erwarten, die am Ende des 21. Jahrhunderts bei etwa $+10 \%$ (Winter) bzw. -10 bis $-20 \%$ (Sommer; je nach Region unterschiedlich) liegen dürfte. Allerdings ist die Bandbreite der Möglichkeiten sehr groß und lässt sowohl stärkere Änderungen als auch unveränderte Niederschläge in allen Saisonen zu. Wenn es zu einer systematischen Änderung kommt, dann wird diese vor allem im Sommer, wahrscheinlich erst in der zweiten Hälfte dieses Jahrhunderts deutlich ausgeprägt sein. Im Jahresmittel ist keine starke Änderung der Niederschlagssumme zu erwarten.

In den folgenden Fallbeispielen werden die Veränderungen des Klimas von Österreich für ausgewählte Landschaftstypen (Gebirge, Stadt, Seen) und Einflussgrößen (Mittelmeer, Wetterlagen) zusammenfassend dargestellt und beschrieben. 
NORDWEST
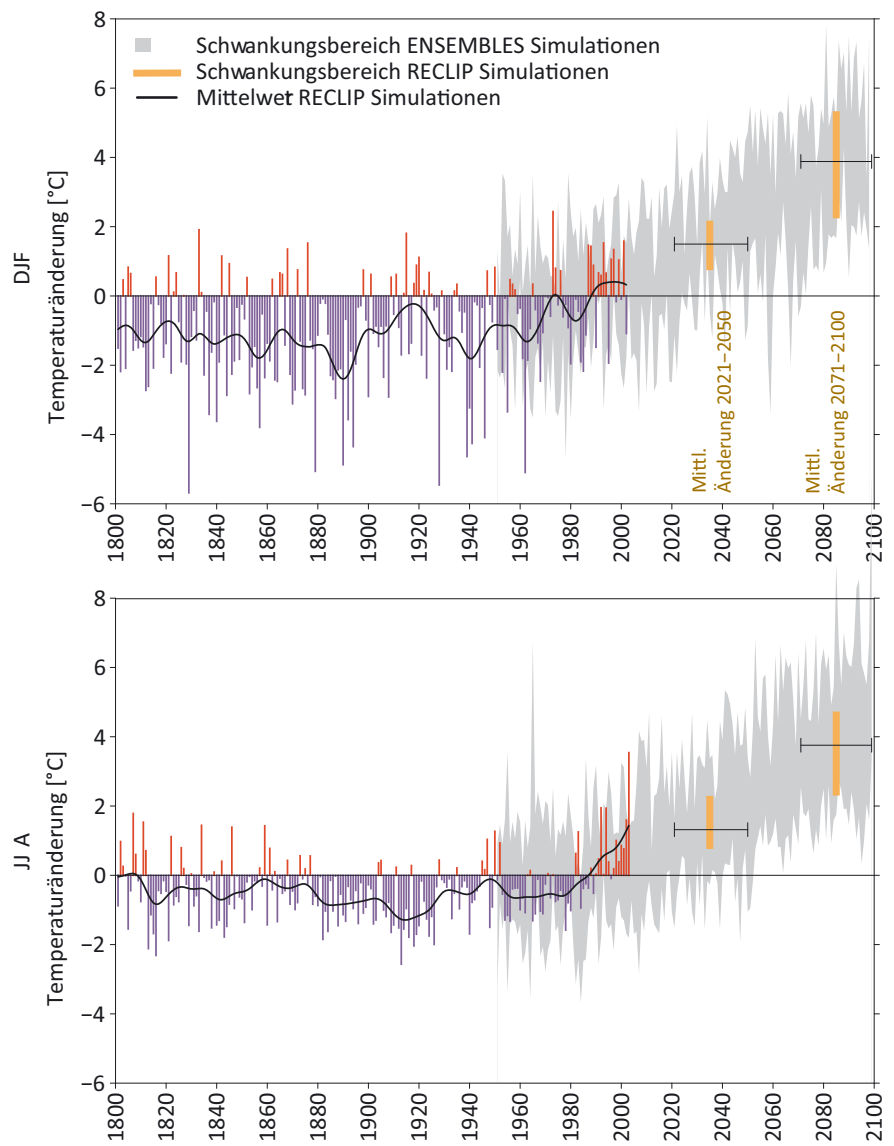

SÜDOST
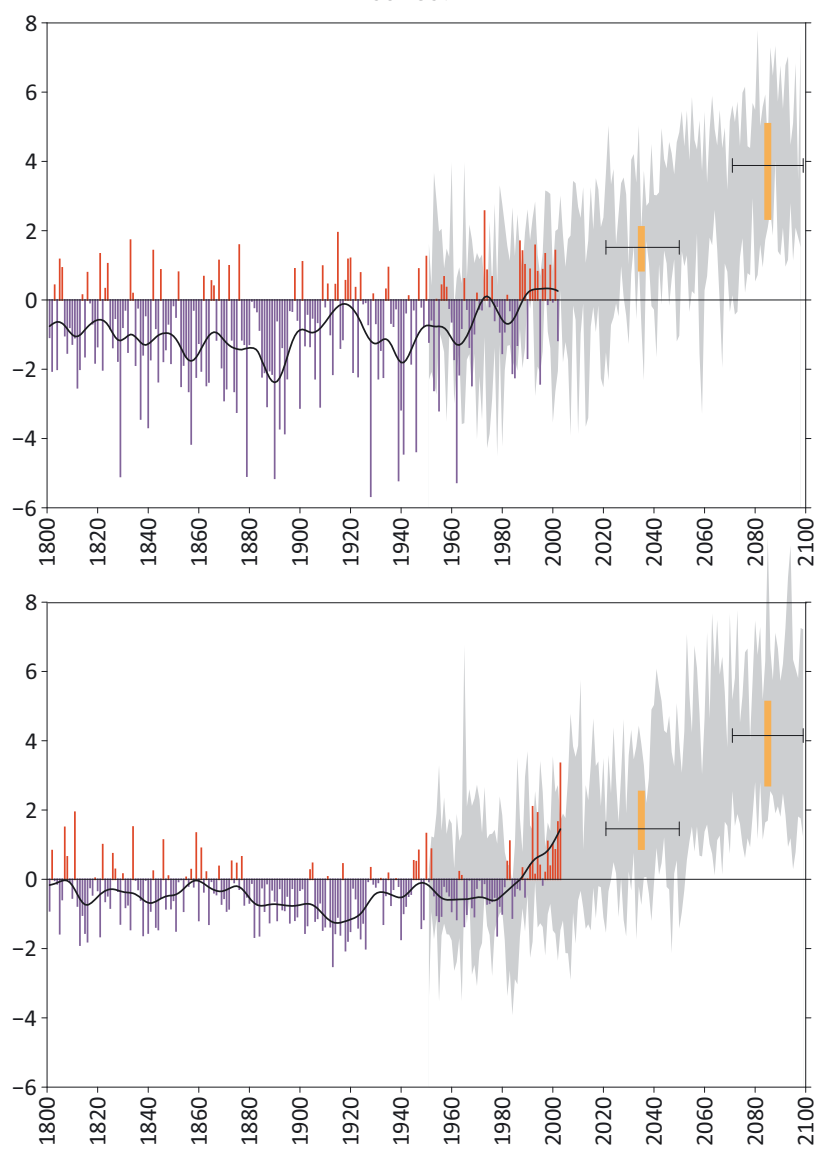

Abbildung 5.1 Temperaturentwicklung in Österreich seit 1800 aus instrumentellen Beobachtungen sowie die zu erwartende Temperaturentwicklung für die Zukunft bis 2100, dargestellt als Abweichung gegenüber dem Mittel 1971-2000. Die Abbildungen oben beziehen sich auf den Winter (DJF), die Abbildungen unten auf den Sommer (JJA), das gesamte Bundesgebiet wurde in zwei Regionen (Nord-West und Süd-Ost) unterteilt. Die Beobachtungsdaten für die Vergangenheit stammen aus der HISTALP Datenbank, die Szenarien für die Zukunft stammen aus 22 Ensembles Simulationen (SRES A1B Szenario, Darstellung als graue Balken für Einzeliahre)' und aus reclip:century (SRES Szenarien A1B, A2 und B1, farbige Balken für die Zeitscheiben 2021-2050 und 2071-2100) ${ }^{2}$

Figure 5.1 Temperature in Austria since 1800 (instrumental observations) and expected temperature development until 2100, shown as a deviation from the mean 1971-2000. Figures at the top show the winter season (DJF), figures at the bottom the summer season (JJA); Austria was divided into two regions (north-west and south-east). The observational data for the past come from the HISTALP database, scenarios for the future from the 22 ensemble simulations (SRES scenario A1B, grey bars for single years) ${ }^{1}$ and from reclip:century (SRES scenarios A1B, A2 and B1, coulored bars for the time slices 2021-2050 and 2071-2100) ${ }^{2}$

\footnotetext{
www.ensembles-eu.org

2 http://reclip.ait.ac.at/reclip_century
}

\subsection{Fallbeispiele: Vergangenes und künftiges Klima in Österreich \\ 5.4 Case study: past and future climate in Austria}

\subsubsection{Klimaönderung im Gebirge - Ursachen und Wirkungen am Beispiel des Sonnblicks}

Das Sonnblickobservatorium (3106 m Seehöhe) in den Hohen Tauern hat eine herausragende Stellung in der österrei- chischen Klimaforschung (Schöner et al., 2012). Mit den mehr als 125-jährigen Klimazeitreihen und einem weit über die meteorologischen Standardmessungen hinausgehenden atmosphärischen Monitoringprogramm können Veränderungen des Hochgebirgsklimas in Österreich für verschiedene meteorologische Zustandsgrößen gezeigt werden. Die umfangreichen Messungen erleichtern das Verständnis für die Ursachen der Klimaänderung, also Klimaantriebe und eventuelle Rückkoppelungsprozesse. Weitere besondere Werte der Hochgebirgsstationen liegen in ihrer Repräsentativität für große Gebiete sowie der Möglichkeit durch Vergleich mit Tieflandstationen Höhenabhängigkeiten der Klimaänderung zu 

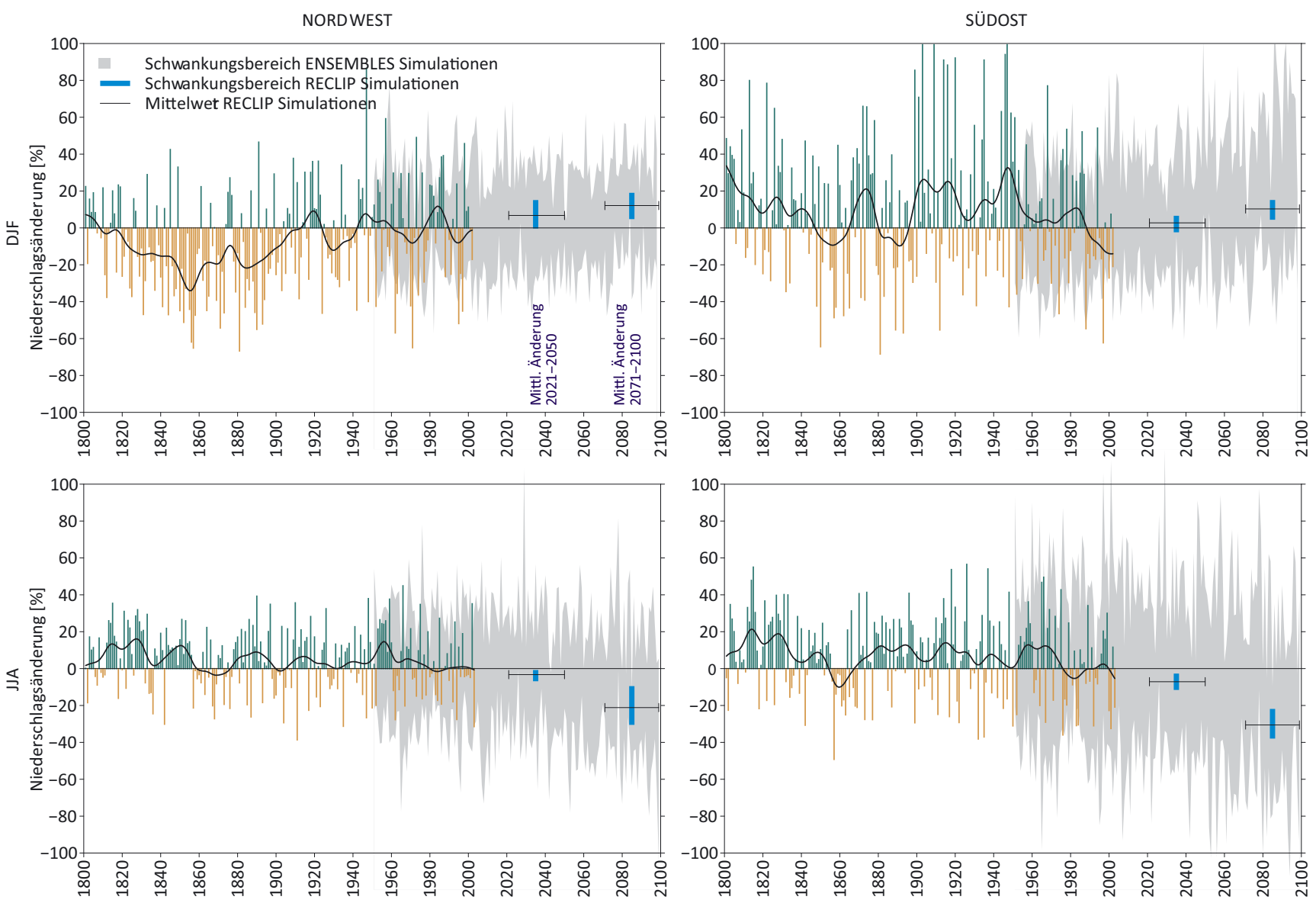

Abbildung 5.2 Niederschlagsentwicklung in Österreich seit 1800 aus instrumentellen Beobachtungen sowie die zu erwartende Niederschlagsentwicklung für die Zukunft bis 2100, dargestellt als Abweichung gegenüber dem Mittel 1971-2000). Die Abbildungen oben beziehen sich auf den Winter (DJF), die Abbildungen unten auf den Sommer (JJA), das gesamte Bundesgebiet wurde in zwei Regionen (Nord-West und SüdOst) unterteilt. Die Beobachtungsdaten für die Vergangenheit stammen aus der HISTALP Datenbank, die Szenarien für die Zukunft sind aus 22 Ensembles Simulationen (SRES A1B Szenario, Darstellung als grave Balken für Einzeliahre) ${ }^{3}$ und aus reclip:century (SRES Szenarien A1B, A2 und B1, farbige Balken für die Zeitscheiben 2021-2050 und 2071-2100) ${ }^{4}$

Figure 5.2 Precipitation development in Austria since 1800 (instrumental observations) and expected development for until 2100 , shown as a deviation from the mean (1971-2000). Above figures show the winter season (DJF), the figures at the bottom the summer season (JJA), the region of Austria has been divided (north-west and south-east) into two regions. The observational data for the past come from the HISTALP database, scenarios for the future of the 22 ensemble simulations (SRES scenario A1B, grey bars for single years) ${ }^{3}$ and from reclip: century (SRES scenarios A1B, A2 and B1, coloured bars for the time slices 2021, 2050 and 2071-2100) ${ }^{4}$

3 www.ensembles-eu.org

4 http://reclip.ait.ac.at/reclip_century

untersuchen. So zeigt etwa eine Untersuchung von Böhm et al. (1998), dass die Temperaturentwicklung der Hochgebirgsstationen der Alpen parallel zu jener der Tieflandstationen ist und auch mit der Entwicklung der Luftdruckänderung (Abnahme der Luftdichte und entsprechende Hebung der Druckniveaus) übereinstimmt. Es ist keine Höhenabhängigkeit der Temperaturtrends aus den instrumentellen Messungen erkennbar. Auer et al. (1998) zeigen eine wesentlich stärkere Zunahme der Sonnenscheindauer an Hochgebirgsstationen im Vergleich zu Tieflandstationen seit ca. 1950 und begründen das mit der zunehmenden Trübung der Atmosphäre durch zunehmende industrielle Prozesse.

Der im Sommer besonders augenfällige Temperaturanstieg am Hohen Sonnblick seit dem Beginn der 1980er Jahre (ca. $+2{ }^{\circ} \mathrm{C}$ ) geht einher mit einer deutlichen Zunahme der Sonnenscheindauer (Abbildung 5.3) und einer Erhöhung der Globalstrahlung - d.h. mit Veränderungen des primären Klimaantriebes. Dabei wird der mehrfache Einfluss des Menschen auf das Klima deutlich erkennbar: So ist zwar die Zunahme der Sonnenscheindauer mit dem generellen Anstieg des Luftdrucks 

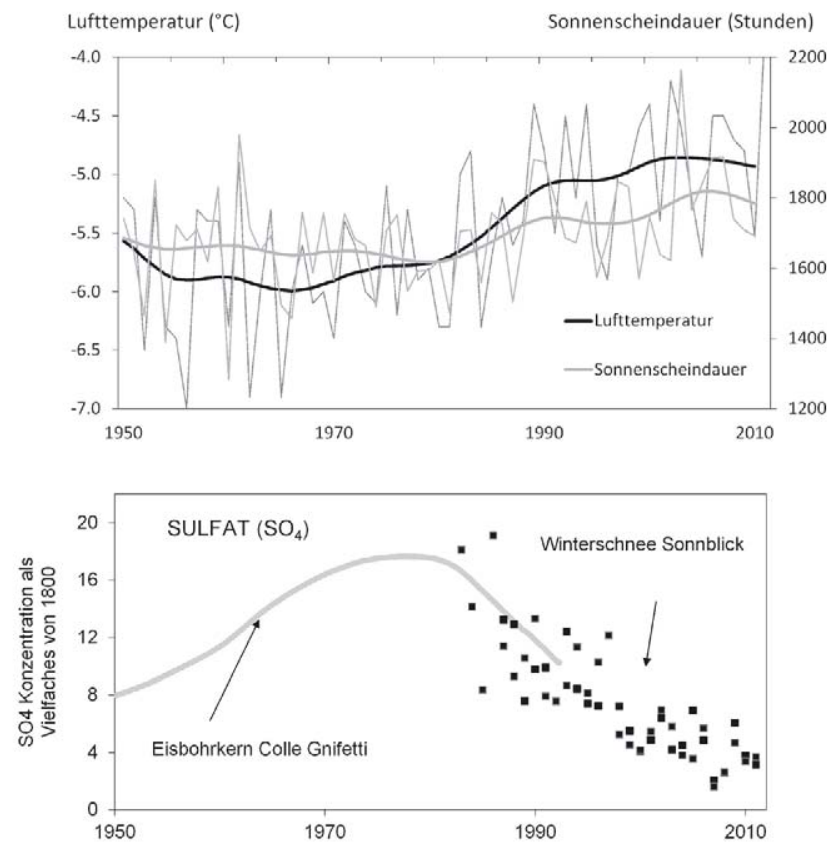

Abbildung 5.3 Oben: Zeitreihen der Jahresmitteltemperaturen (schwarze Linie) und der Jahressummen der Sonnenscheindaver (graue Linie) am Sonnblick für die letzten 60 Jahre sowohl für Einzeljahre (dünne Linie) als auch als gefilterte Zeitreihe (30iähriger Gaußfilter, dicke Linie). Datenquelle: ZAMG

Unten: Zeitreihen der mittleren Sulfatkonzentration in der Schneedecke am Sonnblick für die letzten 30 Jahre (schwarze Punkte) und Sulfatkonzentrationen an einem Firnkern vom Colle Gnifett in der Schweiz (grave Linie). Datenquellen: ZAMG, TU Wien, D. Wagenbach, persönliche Mitteilung

Figure 5.3 Top: Time series of annual air temperatures (black line) and annual hours of bright sunshine (grey line) measured at Sonnblick in the last 60 years, for individual years (thin line) and filtered values (30-years Gaussian filter, bold line) (Datasource: ZAMG) Bottom: Time series of sulphate concentration in the snow cover for the last 30 years (black dots) and sulphate concentrations in a firn core from Colle Gnifetti in Switzerland (grey line). Data source: ZAMG, TU Wien, D. Wagenbach, personal communication

über dem Alpenraum durch einen Nordwärts-Verschiebung des subtropischen Hochdruckgürtels (siehe Band 1, Kapitel 3) zu begründen, der deutliche Anstieg der Globalstrahlung, der die Zunahme der Lufttemperatur mitverursacht, ist jedoch auch eine Folge des sogenannten „Global Brightening“" (siehe z. B. Wild et al. 2005, Ruckstuhl et al., 2008). Maßnahmen zur Reinhaltung der Luft haben zur Abnahme insbesondere der Anzahl an Sulfataerosolen in der Luft geführt, und damit die Streuung der Sonnenstrahlung verringert. Es ist dies die Gegenentwicklung zum „Global Dimming“. Die lange Zeitreihe der Sulfatmessungen in der Schneedecke am Sonnblick belegt dies sehr eindrucksvoll (Abbildung 5.3).

Aber der Einfluss des Menschen äußert sich natürlich vorwiegend durch die Wirkung der Spurengase auf die langwellige terrestrische Strahlung. Die Messungen vom Sonnblick

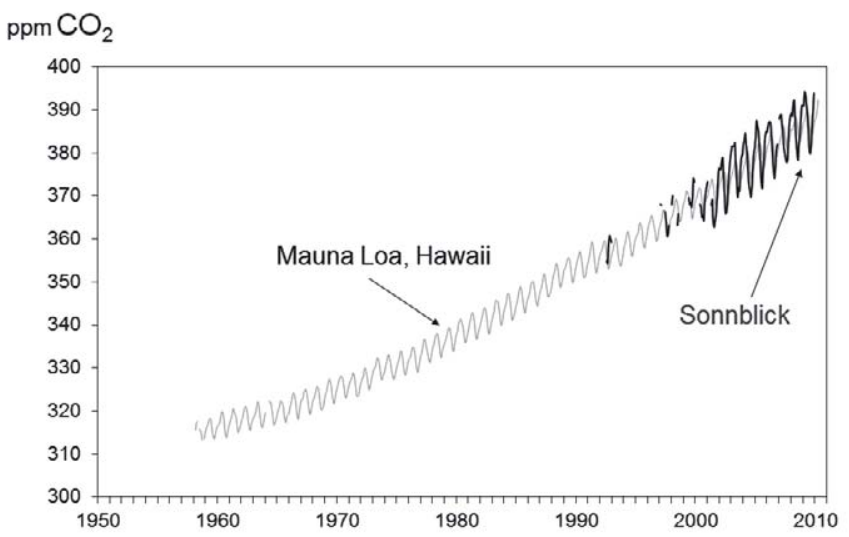

Abbildung 5.4 Zeitreihen des $\mathrm{CO}_{2}$ am Sonnblick (schwarze Linie) im Vergleich zu den Messungen auf Mauna Loa (graue Linie) für die letzten 50 Jahre. Datenquelle: Böhm et al. (2011)

Figure 5.4 Time series of $\mathrm{CO}_{2}$ at Sonnblick (black line) in comparison to the measurements at Mauna Loa (grey line) for the last 50 years. Source: Böhm et al. (2011)

zeigen den verursachenden deutlichen Anstieg des $\mathrm{CO}_{2}(\mathrm{Ab}-$ bildung 5.4). Aus dem Vergleich der $\mathrm{CO}_{2}$-Messungen des Sonnblicks mit der berühmten Messreihe von Mauna Loa auf Hawaii kann man gut erkennen, dass der $\mathrm{CO}_{2}$ Anstieg ein globales Problem ist - die lange Lebensdauer der $\mathrm{CO}_{2}$-Moleküle in der Atmosphäre und die Durchmischung der Atmosphäre führen zu einem parallelen Anstieg der Zeitreihen.

Einen besonders eindrucksvollen Beleg für Klimaänderungen in den Alpen liefern die Gletscher. Der oben beschriebene, deutliche Anstieg der Globalstrahlung und die damit einhergehende starke Zunahme der Lufttemperatur im Sommer führen zu einen deutlichen Zunahme der sommerlichen Gletscherschmelze und stark negativen Massenbilanzen (Abbildung 5.5 unten). Die durch den Temperaturanstieg mitverursachte Abnahme der sommerlichen Schneefälle führt gleichzeitig zu verminderter Albedo und damit zu einer Verstärkung des Schmelzens. Die Folgen für die Hydrologie werden in Band 2 beschrieben. Spezifische Untersuchungen zum Sonnblick finden sich z. B. in Koboltschnig et al., 2009, Schöner und Böhm, 2007. Die in Abbildung 5.5 (oben) gezeigte, deutliche Zunahme des Regenniederschlags am Sonnblick ist ebenfalls für die Gletscher ungünstig, und darüber hinaus eine Ursache für den deutlichen Rückgang des Permafrostes am Sonnblick (Schöner et al., 2012). Das Sonnblickobservatorium ist nicht nur ein guter Standort für die Erfassung von Änderungen des Phänomens Permafrost sondern auch unmittelbar selbst betroffen vom Klimawandel. Für die Absicherung der vom schwindenden Permafrost bedrohten Standfestigkeit des Sonnblickgipfels waren zwischen 2003 und 2006 umfangreiche und kostspielige Stabilisierungsmaßnahmen notwendig. 


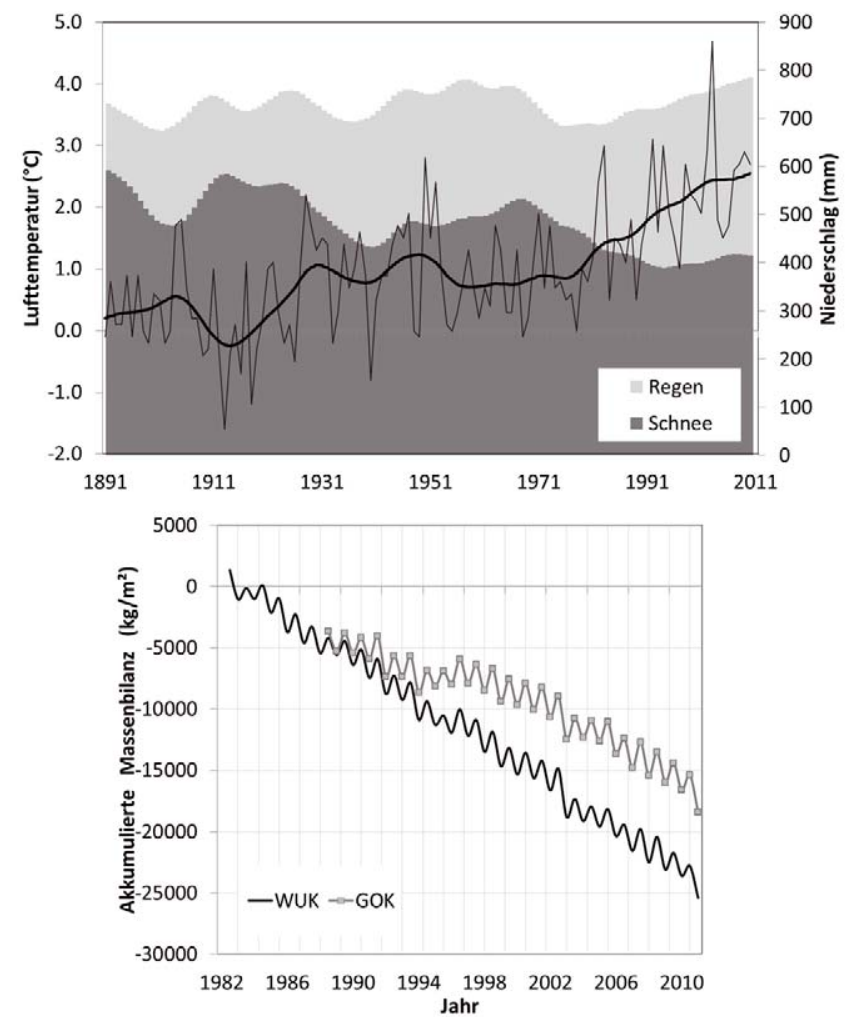

Abbildung 5.5 Oben: Zeitreihe der Lufttemperatur (schwarze Linie, dünn=Einzeljahre, dick=30jährig mittels Gaußfilter gefilterte Werte), des Niederschlags getrennt nach Regen (hellgrau) und Schnee (dunkelgrau) jeweils für den Sommer seit 1891. Unten: Akkumulierte Massenbilanzen der naheliegenden Gletscher Wurtenkees und Goldbergkees. Datenquelle: ZAMG

Figure 5.5 Top: Time series of air temperature (black line, thin $=$ single years, bold $=30$ year Gaussian filtered values), of precipitation split into rain (light grey) and snow (dark grey) for the summer season since 1891. Bottom: Cumulative mass balance of nearby glaciers Wurtenkees and Goldbergkees. Data source: ZAMG

\subsubsection{Klimaänderung in der Stadt Wien}

\section{Wärmeinsel in Wien}

Städtische Räume werden von den sich verändernden klimatologischen Verhältnissen besonders stark beeinflusst. In dicht bebauten Siedlungsgebieten führen Oberflächenversiegelung und das Fehlen von Grünflächen und somit von Verdunstungsflächen zu einer höheren Durchschnittstemperatur. Dieser Effekt wird durch die hohe Wärmespeicherkapazität vieler Baumaterialien und zusätzliche Wärmequellen in Siedlungen, Gewerbe und Industrie verstärkt. Das führt zum Phänomen der sogenannten städtischen Wärmeinsel („Urban Heat Island"), welche als Temperaturunterschied zwischen der Stadt und der ländlichen Umgebung definiert ist (z. B. Oke, 1982; 1987). Der Wärmeinseleffekt ist von der Stadtstruktur und der Topographie abhängig und ist dem regionalen Klima überla- gert. Die Auswirkungen der Städte auf das Klima reichen aber über die Beeinflussung der Temperatur hinaus und zeigen sich auch in der Strahlungs- und Wärmebilanz, den Strömungsund Feuchtigkeitsverhältnissen sowie der Luftqualität.

Die beobachtete Temperaturerhöhung in Wien ist sowohl durch die Erwärmung des regionalen Klimas (Auer et al., 2003, 2007) als auch durch den verstärkten Wärmeinseleffekt zu erklären. Im Jahresmittel war im Zeitraum 1951-1980 die Temperatur des Stadtzentrums von Wien um $1{ }^{\circ} \mathrm{C}$ bis $1,5^{\circ} \mathrm{C}$ höher als jene der Umgebung (Auer et al., 1989). Seit 1951 kann ein statistisch signifikanter Trend steigender Temperaturunterschiede zwischen der Stadt und dem Umland festgestellt werden (Böhm, 1979), der für die Periode 1951-1995 bis zu $0,6^{\circ} \mathrm{C}$ (für die gesamte Periode) an einzelnen Stationen in Wien beträgt (Böhm, 1998). Als Ursache wurden Änderungen in der städtischen Morphologie und im Energieverbrauch genannt.

Weiters zeigen Messdaten der Lufttemperatur von Wien für die vergangenen Jahrzehnte eine Zunahme der thermischen Belastung im Sommer (Abbildung 5.6). Die Häufigkeitsverteilung der Tagesmaxima der Temperatur in den Sommermonaten hat sich deutlich zu höheren Temperaturen verschoben, ohne dass sich dabei das kalte Ende der Verteilung wesentlich verändert hätte.

Die Anzahl der Sommertage (Tage an denen die Maximumstemperatur größer oder gleich $25^{\circ} \mathrm{C}$ ist) hatte ein Minimum während des ersten Weltkrieges mit weniger als 30 Sommertagen pro Jahr. In der ersten Dekade des 21. Jahrhunderts lag der Mittelwert der Sommertage in Wien (Station Hohe

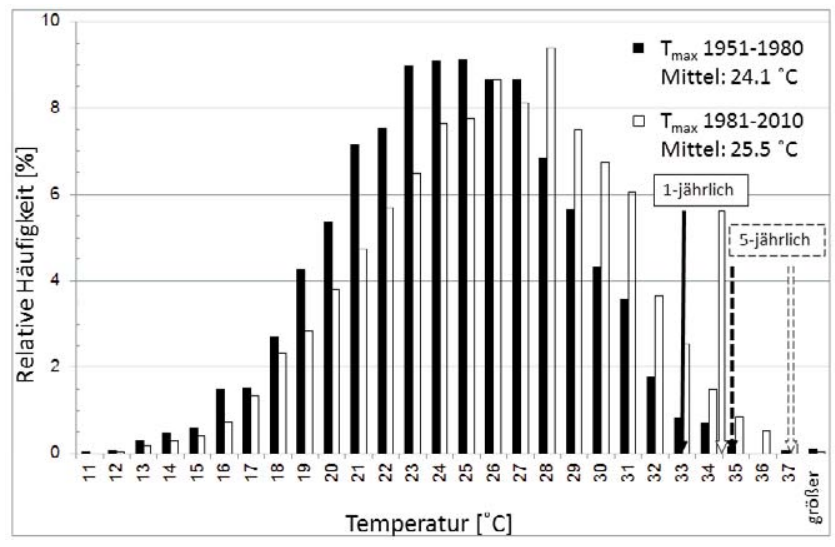

Abbildung 5.6 Häufigkeitsverteilung der Maximalen Sommertemperaturen an der Station Wien, Hohe Warte, in den Monaten Juni, Juli und August für die Zeiträume 1951 bis 1980 und 1981 bis 2010. Datenquelle: ZAMG

Figure 5.6 Frequency distribution of maximum of summer temperatures for station Wien, Hohe Warte, for the months June, July and August in 1951-1980 versus 1981-2010. Data source: ZAMG 


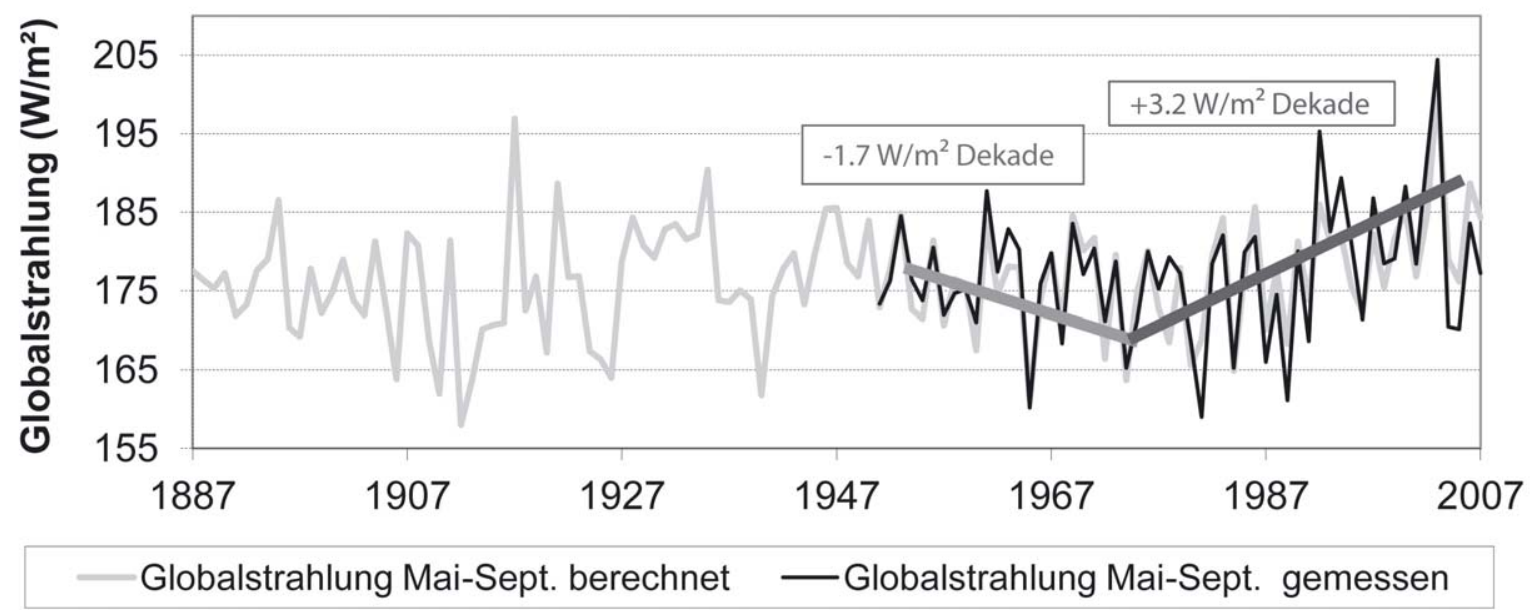

Abbildung 5.7 Zeitlicher Verlauf der Globalstrahlung für die Periode Mai-September an der Station Wien, Hohe Warte, aus Messungen seit 1951 und mittels empirischer Formel berechnet aus der Sonnenscheindaver. Datenquelle: ZAMG

Figure 5.7 Global radiation averaged over the period May-September at station Vienna, Hohe Warte, from measurements since 1951 as well as empirically estimated from sunshine duration. Data source: ZAMG

Warte) bereits bei 64 Tagen pro Jahr. Im klimatologisch außergewöhnlichen Jahr 2003 wurden sogar über 100 Sommertage registriert.

Noch deutlicher wird die Auswirkung der Temperaturzunahme anhand der Häufigkeit von Hitzetagen, also Tagen mit Temperaturen über $30^{\circ} \mathrm{C}$, die im Zeitraum 1910 bis 2000 von ca. 2 auf 17 Tage gestiegen sind (Auer et al., 2003). Fünfjährliche Hitzeereignisse der Periode 1951 bis 1980 sind in der Periode 1981 bis 2010 zu einjährlichen geworden (vgl. Abbildung 5.6). Hitzetage werden jedoch nicht nur häufiger, sondern sie treten an der Station Wien Hohe Warte mit vergleichbarer Wahrscheinlichkeit auch etwa 15 Tage früher im Jahr auf (Piribauer und Strasser, 2010). Trotz der deutlichen Zunahme der Mittel- und Maximumstemperaturen seit ca. 1970 zeigt sich keine parallele Abnahme in der Anzahl der Frosttage (Auer et al., 2003).

Der Wärmeinseleffekt führt zu hohen Wärmebelastungen in den städtischen Räumen. Die nächtliche Abkühlung in Städten nimmt mit zunehmenden Temperaturen ab. In Wien stieg in der Periode 1985 bis 2005 die Zahl der Nächte, in denen die Temperatur nicht unter $16^{\circ} \mathrm{C}$ gesunken ist, gegenüber der Referenzperiode 1961 bis 1990 um ein Drittel - von 30 auf 40 Nächte (Formayer et al., 2007). Die nächtliche Abkühlung ist während Hitzeperioden ein wesentlicher Faktor für die Erholung des Menschen von übermäßiger Wärmebelastung während des Tages (Thaler et al. 2005, 2008). Weitere Ausführungen zu den Auswirkungen erhöhter Temperaturen auf die Gesundheit finden sich im Band 2, Kapitel 6 und Band 3, Kapitel 5.

Besonders auffällig ist eine deutliche Zunahme der Sonnenscheindauer und Globalstrahlung in Wien seit ca. 1980 (Abbil- dung 5.7). Das beobachtete „Global Brightening“ (Wild et al., 2005, Ruckstuhl et al., 2008) kann als Erklärung für die deutliche Zunahme der Globalstrahlung herangezogen werden.

\section{Klimazukunft und Auswirkungen}

Für die großen österreichischen Städte, insbesondere Wien, ist die infolge des Klimawandels zunehmende Hitzebelastung als eine wesentliche Herausforderung zu sehen. Die Analyse von Klimaszenarien aus regionalen Multimodell-Simulationen (Schär et al., 2004; Fischer und Schär, 2010) zeigt eine Erhöhung der Intensität und Häufigkeit der Hitzewellen in ganz Europa. Für Wien wurde mittels statistischem Downscaling ein Anstieg von $46 \%$ auf $69 \%$ Sommertage und von $10 \%$ auf $24 \%$ Hitzetage bis Ende des Jahrhunderts im Vergleich zu Klimanormalperiode 1961 bis 1990 errechnet (Formayer et al., 2009). Berechnungen, die auf dem regionalen REMOUBA Klimamodell (Jacob et al., 2008) beruhen, zeigen für die Station Hohe Warte bis zur Mitte des Jahrhunderts einen moderaten Anstieg, danach aber eine deutlich raschere Zunahme hoher Temperaturen. In der Periode 2070 bis 2100 liegen die Werte bei den Hitzetagen im Mittel bei mehr als 35 Tagen, der 10-Perzentilwert bei nahezu 60 Tagen für das SRES A1B Szenario (Formayer et al., 2007). Andere, mit denselben Modellannahmen berechnete Temperaturindizes bestätigen verstärkte Auswirkungen des Klimawandels in der zweiten Hälfte des 21. Jahrhunderts. Bemerkenswert ist die Zunahme der sogenannten Kyselytage, einem Indikator für Hitzeperioden (Kyselý et al., 2000) und heiße Nächte (Temperaturen sinken nachts nicht unter $\left.18^{\circ} \mathrm{C}\right)$. Erstere versechsfachen sich etwa in der Pe- 

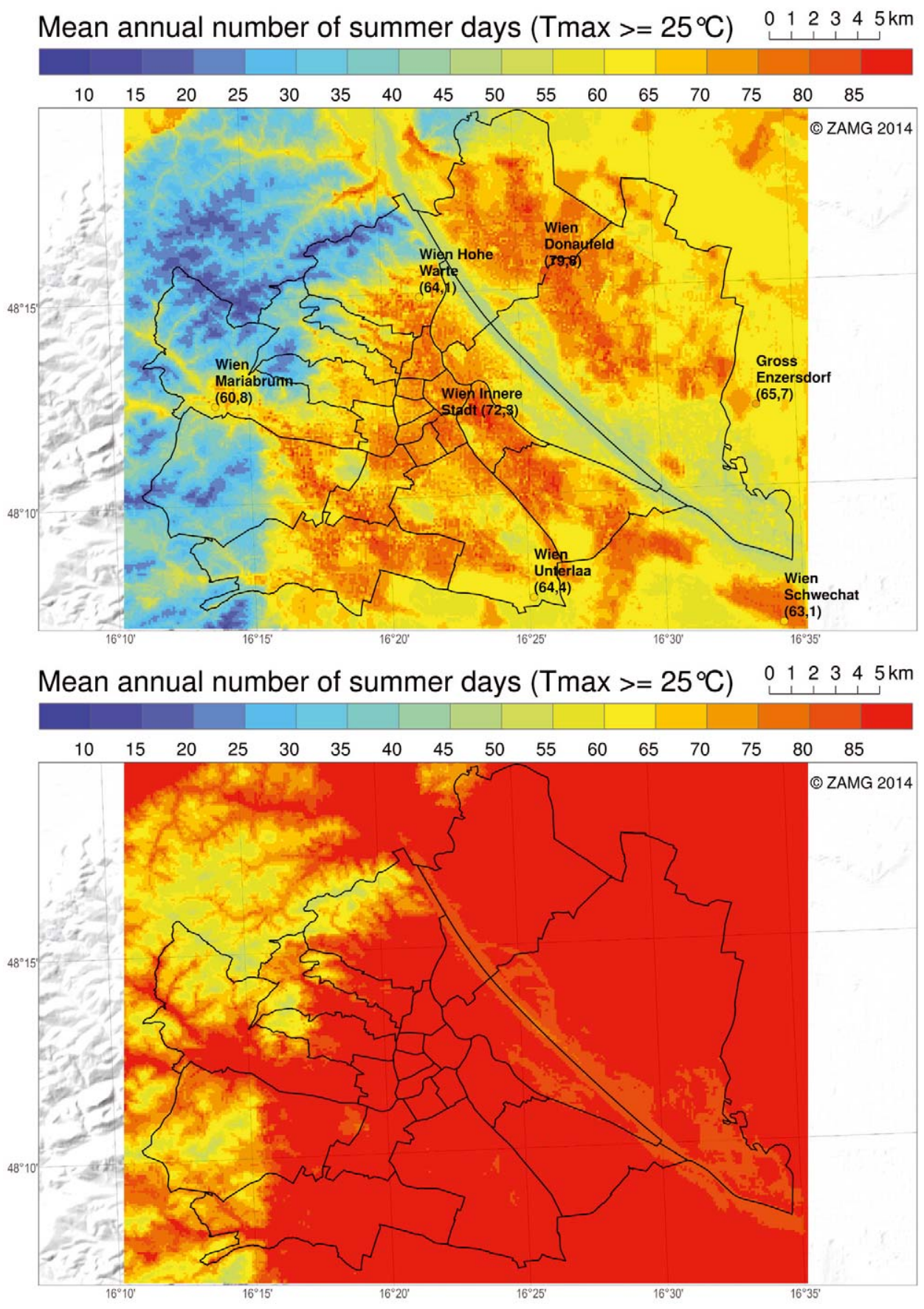

Abbildung 5.8 Mittlere Anzahl der Sommertage in Wien und Umgebung für die Zeitraum 1981 bis 2010 (links, beobachtet) und 2071 bis 2100 (rechts, mittels Stadtklimamodell MUKLIMO_3, Zuvela-Aloise, 2011). Zugrundeliegendes Klimaszenario: CosmoCLM basierend auf ECHAM5 IPCC Szenario A1B, Lautenschlager et al. $(2009,2011)$

Figure 5.8 Mean number of summer days for the area of Vienna and surroundings for the period 1981-2010 (left, from observations) and 2071-2100 (right, modelled with model MUKLIMO_3, Zuvela-Aloise, 2011). Underlying climate scenario: Cosmo-CLM based on ECHAM5 IPCC Scenario A1B, Lautenschlager et al. (2009, 2011)

riode 2070 bis 2100 im Vergleich zur Periode 1960 bis 1990 , letztere verzehnfachen sich sogar. In beiden Fälle ist der primäre Anstieg in der 2. Hälfte des Jahrhunderts zu verzeichnen.

In der zweiten Hälfte des 21. Jahrhunderts könnte ohne Anpassungsmaßnahmen die hitzebedingte Mortalität um 129 \% (Muthers et al., 2010) bis 200 \% (Moshammer et al., 2006) gegenüber jener Ende des 20. Jahrhunderts steigen, vor allem durch extreme Hitze. Von der Hitzesterblichkeit besonders betroffen sind Frauen, ältere Menschen und Personen in „ärmeren“ Bezirken. Das Sterberisiko unter Hitzeeinwirkung erwies sich für KrankenhauspatientInnen und für HeimbewohnerInnen gleich hoch wie für andere Personen; es dürften sich zwei Effekte überlagern - zum einen sind Menschen, die sich in Krankenhäusern und Heimen befinden großteils a priori geschwächt und daher für Zusatzbelastungen anfälliger, andererseits trägt das geschulte Personal in Krankenhäusern und Heimen zur Minderung des Risikos bei (Moshammer et al., 2009). Eine klare Schwelle, ab welcher die Sterblichkeit stärker ansteigt und daher Anpassungsmaßnahmen zu setzen wären, konnte nicht gefunden werden. 
Die Indikatoren für den Energiebedarf zur Heizung (Heizgradtage, ÖNORM 8135) bzw. Kühlung (Kühlgradtage, Sailor, 2001) im Wohnbereich zeigen erwartungsgemäß gegenläufiges Verhalten: Die Heizgradtage sinken um etwa 30 \%, die Kühlgradtage erhöhen sich um mehr als einen Faktor 2,5 bis zur Periode 2070 bis 2100 (Formayer et al., 2007, Prettenthaler und Gobiet, 2008).

Für die Biosphäre relevante abgeleitete Größen, wie etwa die Tage der Vegetationsperiode (FAO, 1996) oder die Gradtagssummen (Harlfinger und Knees, 1999) zeigen vergleichsweise geringe Änderungen seit 1960, lassen aber eine deutliche Zunahme in der 2. Hälfte dieses Jahrhunderts erwarten. Für die größte Grünfläche Wiens, den Wienerwald, ist im zeitlichen Verlauf ein klimabedingter Produktivitätsrückgang zu erwarten. Andere Vulnerabilitätsindikatoren, wie Vorrat oder Kohlenstoffspeicherung weisen geringere Veränderungen als die Produktivität auf und werden durch das jeweilige Bewirtschaftungskonzept stärker beeinflusst als durch den Klimawandel. Durch Anpassung der Bewirtschaftungsform können voraussichtlich die erwünschten Ökosystemleistungen im Biosphärenpark Wienerwald unter den erwarteten Klimawandelbedingungen aufrechterhalten werden (Schörghuber et al., 2012).

Die Landnutzungsmuster der Stadt (z. B. Verteilung von Grünflächen, Wasserflächen, Bebauungsdichte) bestimmen die räumliche Verteilung der zu erwartenden Wärmebelastung (Abbildung 5.8). In Zuvela-Aloise (2011) wurde ein Ensemble von Klimaprojektionen für die Stadt Wien mittels des Stadtklimamodells MUKLIMO_3 (Sievers, 1990; 1995) und der sogenannten Quadermethode (Früh et al, 2011a,b) berechnet, um die flächenhafte Verteilung der Wärmebelastung in Wien zu untersuchen. Die Ergebnisse zeigen für den Zeitraum 2021 bis 2050 wenig Änderung bis zu einer mäßigen Zunahme der mittleren jährlichen Anzahl der Sommertage (bis zu 20 Sommertage mehr). Bis Ende des Jahrhunderts ist aber (in Abhängigkeit des betrachteten Klimaszenarios) mit einer Zunahme von 20 bis 50 Sommertagen und mit einem größeren Anstieg der Hitzebelastung zu rechnen. Die räumliche Verteilung bleibt erhalten - die niedrigsten Temperaturen finden sich jeweils im Bereich des Wienerwaldes, des letzten Ausläufers der Alpen, und im unmittelbaren Donautal. Die meisten heißen Tage treten in den dicht verbauten inneren Bezirken und im flachen, schon pannonisch beeinflussten Osten Wiens auf.

Niederschlagsszenarien für den Raum Wien gehen ana$\log$ der Entwicklung im Nordosten Österreichs von einer Abnahme des Niederschlags im Sommer und einer Zunahme im Winter aus, die umso ausgeprägter ausfallen, je ext- remer die Klimaszenarien sind (Damerau, 2008). Die Szenarienergebnisse für Strahlung und Wind sind mit wesentlich größeren Unsicherheiten behaftet, und insbesondere letztere sind auch stark von der Stadtentwicklung und Bebauung abhängig.

Modellexperimente in denen der Anteil der städtischen Parkflächen, der Wasserflächen oder der Bebauungsstruktur künstlich verändert wurden, zeigen deutlich den Einfluss der Landnutzung auf das Stadtklima von Wien (Zuvela-Aloise et al., 2013). Die dadurch veränderte Verdunstung und Albedo verändern die Strahlungsbilanz und die Windgeschwindigkeit. Dies hat beispielweise deutliche Auswirkungen auf die Anzahl der modellierten Sommertage (Zuvela-Aloise et al., 2013). Optionale städtebauliche Maßnahmen können damit recht gut auf ihre Klimawirksamkeit überprüft werden. Weitere Modellexperimente und Modellverbesserungen sind jedoch notwendig um die Ergebnisse operationell in die Stadtplanung einfließen lassen zu können.

Grün- und Freiräume können nicht nur dem Temperaturanstieg entgegenwirken. Sie können auch dazu beitragen, die Widerstandsfähigkeit von Städten gegenüber Extremereignissen zu steigern. Sie liefern mit ihrer abkühlenden und Wasser zurückhaltenden Wirkung bzw. verdunstenden Funktion einen zentralen Beitrag zum Temperatur- und Wasserhaushalt in der Stadt (Meinharter und Balas, 2011). Der Städtetourismus weist in Wien in den Sommermonaten (Juli, August) die höchste Anzahl an TouristInnen auf. Ein wachsender Anteil der TouristInnen gehört der Gruppe der 60-79-Jährigen an, die im Allgemeinen besonders hitzeempfindlich sind. Eine Befragung von TouristInnen zeigte, dass vor allem in den Bereichen Begrünung, Information von TouristInnen (z. B. Kennzeichnung von Trinkbrunnen und kühlen Orten in Stadtplänen, Bereitstellung hitzeadäquater Besichtigungstipps in den Unterkünften und über Internet-Applikationen) sowie Weiterbildung von TouristikerInnen Handlungsbedarf gegeben ist (Allex et al., 2011).

Um die Wärmebelastung zu minimieren, ist es notwendig städtebauliche Maßnahmen unter Berücksichtigung klimatologischer Aspekte im Vorfeld einzuplanen (Kromp-Kolb et al., 2007; Mursch-Radlgruber et al., 2009; Loibl et al., 2011; Zuvela-Aloise et al., 2013). Aus meteorologischer Sicht sollten klimagerecht geplante Städte der Zukunft u. a. folgende Eigenschaften aufweisen:

- Gute Wärmedämmung der Gebäude und ausreichend Verschattungsmöglichkeiten;

- Begrünung von Dach-, Fassadenflächen und Straßenraum zur Reduktion von Oberflächentemperaturen; 


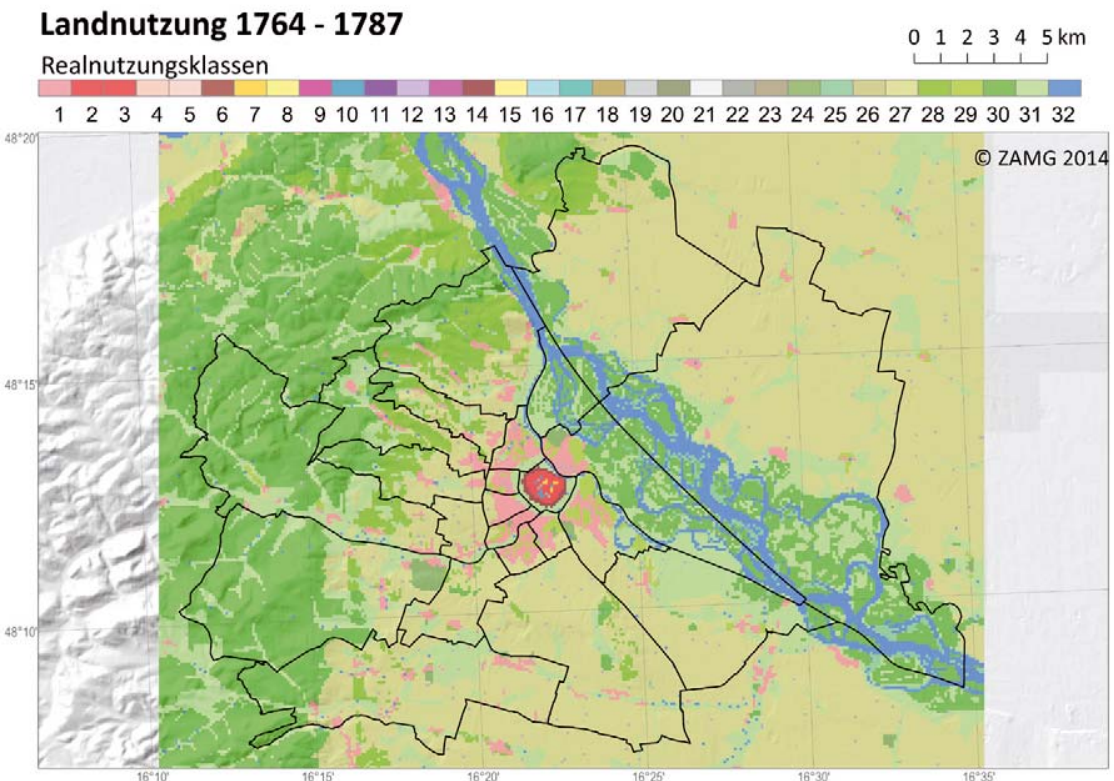

\section{Landnutzung 2007/2008}

Realnutzungsklassen

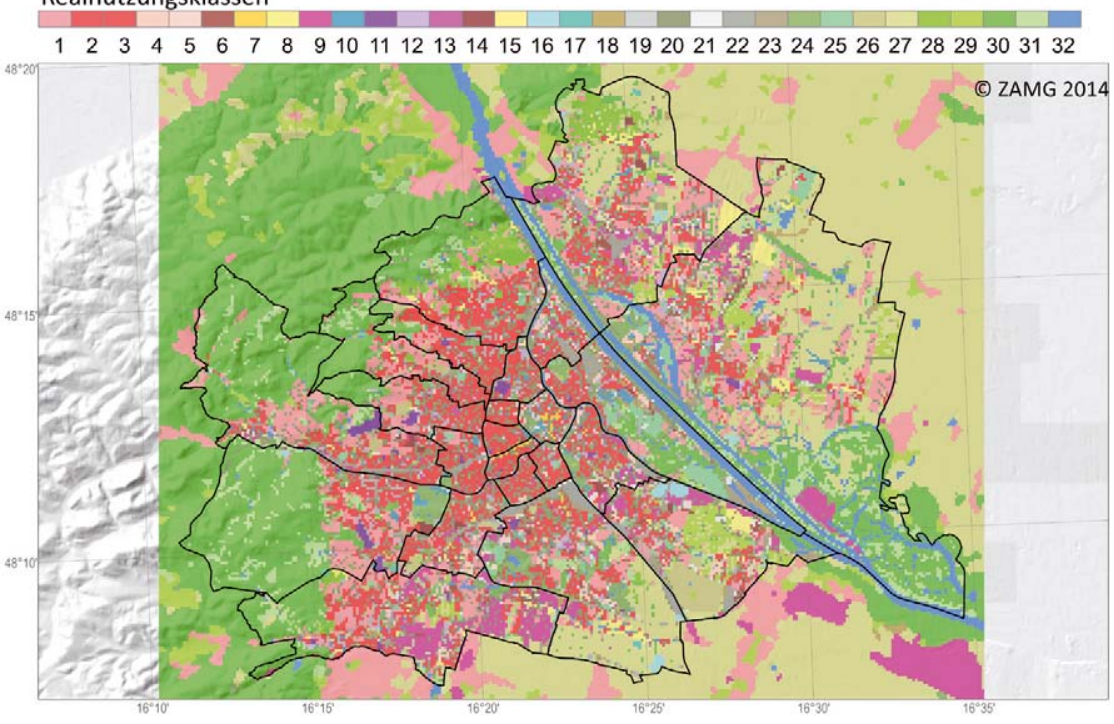

\section{Landnutzung hypothetische Zukunft}

Realnutzungsklassen

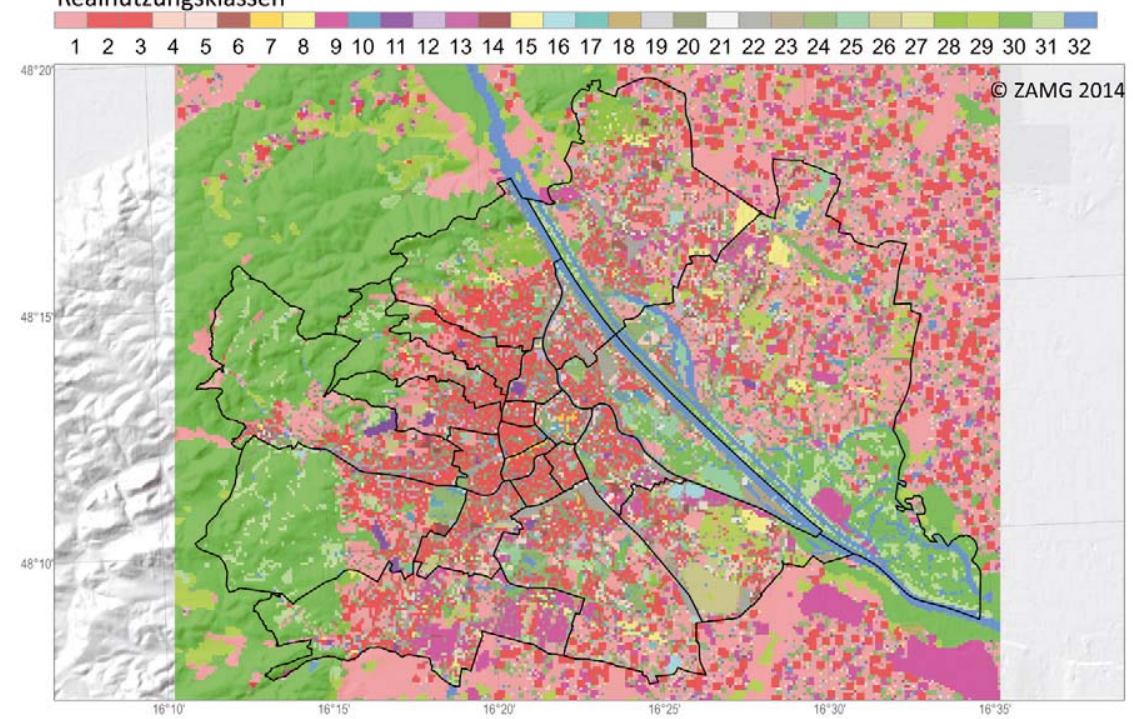

Abbildung 5.9 Landnutzungskarten von Wien und Umgebung. Oben: Digitalisierte historische Landkarte der Ersten Josephinischen Landesaufnahme von 1764 bis 1787 (ÖSTA, Erste Landesausfnahme, Digitalisierung: ZAMG). Mitte: Heutige Landnutzung, erstellt aus Landnutzungsdatensätzen der MA $18^{5}$ und satellitenbasierten Landnutzungsdaten (Steinnocher, 1996). Unten: hypothetische Entwicklung der Stadt mit neven Bebauung in der Süd-Ost Region und Verdichtung der bestehende lockeren (Misch) Bebauung. Die Realnutzungsklassen sind in 4 Typen geteilt: Baulandnutzung (1-18), Straßen und Verkehr (19-23), Grünlandnutzung (24-31) und Wasser (32). Der historische Datensatz unterscheidet 14 Landnutzungsklassen. Quelle: ZAMG

Figure 5.9 Landuse maps of Vienna and surroundings. Top: Digitized historical map of the first topographic survey "Erste Josephinische Landesaufnahme" 1764 to 1787 (ÖSTA, Erste Landesaufnahme, Digitized by ZAMG) Center: Todays landuse derived from landuse data of MA $18^{5}$ and satellite based data (Steinnocher, 1996). Bottom: Hypothetical development of landuse for Vienna area with densification of existing loosely built-up area in the South-East region of the city. Landuse classes are divided into 4 types: built-up areas (1-18), streets and traffic (19-23), vegetation (24-31) and water (32). Historical dataset differentiates 14 landuse classes. Source: ZAMG

5 http://www.stadtentwicklung.wien.at 
- Bei fehlendem Grün, helle Oberflächen zur Erhöhung der Albedo (Reduktion der Absorption von Strahlung);

- Kompakte, aber belüftungswirksame Bebauungsstrukturen.

Detaillierte Ausführungen zu Anpassungsmaßnahmen in Städten finden sich in Band 3.

\section{Klimawandel und Urbanisierung}

Zuzüglich zur regionalen Klimaänderung ist zu erwarten, dass die Entwicklung der Stadt zu weiterer Urbanisierung führt. Aufgrund der Tendenz zur Verdichtung existierender Bebauung und der Urbanisierung der benachbarten, ländlichen Flächen kann sich in Zukunft der Wärmeinsel-Effekt noch verschärfen. Dies kann bedingen, dass die exzessive Hitze in städtischen Bereichen noch größer wird, als aus den Klimaszenarien zu erwarten.

Die städtische Entwicklung Wiens ist in Abbildung 5.9 illustriert. Historische Landkarten der Stadt Wien und Umgebung vom Ende des 18. Jahrhunderts (ÖSTA, Erste Landesaufnahme) zeigen enorme Unterschiede zur heutigen Stadtstruktur. Der historische Kern der Inneren Stadt ist weitgehend unverändert geblieben, aber die Umgebung wurde stark verbaut. Die alte lockere Bebauung wurde verdichtet und die Donau reguliert. Große Teile der ehemalige Weingärten und landwirtschaftlichen Flächen gehören heute zu den suburbanen Wohn(misch)gebieten.

Die Urbanisierung Wiens erfolgt derzeit hauptsächlich am südlichen und östlichen Stadtrand, während der Stadtentwicklungsplan eine Fortsetzung dieses Trends im Osten vorsieht. Die noch zur Verfügung stehenden Flächen in den unmittelbar angrenzenden Gebieten im Süden und im Norden werden in absehbarer Zeit verbaut sein. Wien hatte 2011 eine Einwohnerzahl von 1,75 Mio., das sind $20 \%$ der österreichischen Bevölkerung. Etwa 2030 könnte die 2-Millionengrenze überschritten werden und bis Mitte des 21. Jahrhundert die Bevölkerung auf ca. 2,14 Millionen angewachsen sein (Statistik Austria, 2012). Ein gutes Fünftel davon wäre wahrscheinlich über 65 Jahre alt, eine Bevölkerungsgruppe für die Hitze besonders belastend ist. Die aus dem Klimawandel ableitbare künftige Zunahme der Hitzebelastung könnte sich zu einer besonderen Herausforderung der Städteplanung entwickeln.

Studien zur Entwicklung des Klimas in Wien unter Berücksichtigung der Stadt-, Bevölkerungs- und Wirtschaftsentwicklung liegen derzeit nur in geringer Anzahl vor. Damerau (2008) untersuchte drei mögliche Entwicklungsszenarien für Wien, angelehnt an die SRES-Szenarien A2, A1 sowie B1 und fand trotz nur geringfügig unterschiedlich angenommener
Entwicklungsraten im Energiebereich wesentliche Divergenzen in der Entwicklung. Angesichts der Langfristigkeit städtischer Planungen und der Langlebigkeit von Baumaßnahmen sollten Untersuchungen in diesem Bereich im prioritären Interesse der Stadt Wien liegen. Je später Entscheidungen für eine klimafreundliche Entwicklung fallen, desto schwieriger gestalten sich die erforderlichen Umstellungen, da sie durch früher getroffene Entscheidungen behindert werden können, sogenannte Lock-in Effekte (Nakicenovic, 2003).

\subsubsection{Klimaänderung und Seen - Der Neusiedlersee}

Der Neusiedler See ist einer der wenigen Steppenseen Europas. Er zeichnet sich durch seinen Schilfgürtel, seine geringe Tiefe sowie durch sein mildes und windiges Klima aus. Er bestimmt das Landschaftsbild des östlichen Burgenlandes, begünstigt das regionale Klima und wird durch Tourismus, Wassersport, Schifffahrt und Fischerei genutzt. Der einzigartigen Fauna und Flora dieser historischen Kulturlandschaft tragen die Nationalparks Neusiedlersee-Seewinkel und Fertő-Hanság sowie die Ernennung zum UNESCO-Welterbe mit der Bezeichnung Kulturlandschaft Fertő/Neusiedlersee Rechnung.

Periodisches Austrocknen zählt zu den Charakteristika eines Steppensees. Der Neusiedlersee trocknete im 18. und 19. Jahrhundert je zweimal aus, zuletzt von 1864 bis 1870 . Die anthropogenen Einflüsse auf den Wasserhaushalt des Sees, insbesondere die umfangreichen Drainagemaßnahmen zur Urbarmachung des ehemals weitläufigen Sumpfgebietes im Süden des Sees, führten zu maßgeblichen Änderungen im hydrologischen System.

Durch die Inbetriebnahme einer Wehranlage im Jahr 1965 zur Regulierung der Ableitungsmengen in den Hanságkanal, der den See über das Ikva Rabnitz Flusssystem mit der Mosoni-Donau verbindet, konnte der mittlere Seewasserpegel signifikant angehoben werden. Trotz der menschlichen Eingriffe unterliegt der Wasserhaushalt des Sees im Wesentlichen natürlichen Einflüssen, die stark klimaabhängig sind. Im Mittel stammen $79 \%$ der Wassereinträge in den Neusiedlersee vom Niederschlag, $14 \%$ von der Wulka, $5 \%$ von anderen, kleineren Gewässern, $1 \%$ aus dem Grundwasser und weniger als $1 \%$ aus Abwasseranlagen. Die Verluste des großen, seichten Sees gehen zu $90 \%$ auf Verdunstung zurück und $10 \%$ werden über den künstlichen Kanal abgelassen (Soja et al., 2013).

Das Klima rund um den Neusiedler Sees wird nachweislich von diesem beeinflusst. Der See mit seiner ca. $300 \mathrm{~km}^{2}$ großen Wasserfläche dämpft die Extremwerte der Temperatur. Die mittleren Maxima sind bei den seenahen Stationen im Som- 
mer bis zu $2^{\circ} \mathrm{C}$, im Winter bis zu $1{ }^{\circ} \mathrm{C}$ niedriger, die mittleren Minima dagegen im Sommer bis zu $2^{\circ} \mathrm{C}$ und im Winter bis zu $1{ }^{\circ} \mathrm{C}$ höher. Die Anzahl der Frosttage war an den Uferstationen gegenüber der Umgebung um 10 Tage, die der heißen Tage um 5 Tage vermindert (Dobesch und Neuwirth, 1974; Dobesch und Neuwirth, 1982).

Im Zeitraum 1966 bis 2007 sind Temperaturen und Niederschläge im Gebiet des Neusiedlersees tendenziell gestiegen. Die geringfügig niedrigeren Niederschläge des Zeitraumes 1997 bis 2004 bei steigender Temperatur führten zu kontinuierlich sinkenden Wasserständen. Der niedrige Seewasserstand im Jahr 2003, verursacht durch einen extrem niedrigen Jahresniederschlag und hohe Luft- und Wassertemperaturen, ließ die Frage aufkommen, ob unter zukünftigen Klimabedingungen wieder eine Austrocknung des Sees zu erwarten sei.

Eitzinger et al. (2009) simulierten zur Beantwortung dieser Frage mittels eines hydrologischen Seemodells den Seepegel in Abhängigkeit von meteorologischen Daten. Die Sensitivität hinsichtlich des Jahresniederschlags ist hoch: Bereits geringfügige Änderungen von 5 bis $10 \%$ des Niederschlages haben unter den derzeitigen Klimaverhältnissen einen deutlichen Effekt auf die Auftrittswahrscheinlichkeit von Niedrigwasserständen. Bei einer ständigen Aufeinanderfolge des extrem trockenen Jahres 2003 (ca. $40 \%$ des Jahresniederschlages des Mittels 1961 bis 1990) würde der See nach 4 bis 6 Jahren weitgehend austrocknen. Die Sensitivität wird bei höheren Temperaturen wegen der zunehmenden Verdunstung noch verstärkt.

Berechnungen für Klimaszenarien mit einer mittleren Temperaturerhöhung von $1,9^{\circ} \mathrm{C}$ (2020er Periode) bzw. $2,5^{\circ} \mathrm{C}$ (2040er Periode) mit künstlich generierten Wetterdaten (Wettergenerator) ergaben bei Erwärmung um 2,5 ${ }^{\circ} \mathrm{C}$ eine Erhöhung der Verdunstung um mehr als $20 \%$. Um diesen Wasserverlust zu kompensieren, müssten die Niederschläge ebenfalls um etwa $20 \%$ zunehmen. Vorliegende Klimaszenarien lassen dies als unwahrscheinlich erscheinen. Nach den Modellberechnungen weisen niedrigere Seewasserpegel daher höhere Auftrittswahrscheinlichkeiten auf. Geht man z. B. vom Pegel 115 m Seehöhe ü.A. aus, so wird dieser in der Periode 1961 bis 1990 alle 250 Jahre unterschritten, in der Periode 1991 bis 2004 bereits alle 71 Jahre, in den Szenarien für 2020 und 2040 aber schon alle 7 bzw. 3 Jahre (Eitzinger et al., 2009). Ein wesentliches Ergebnis dieser Analysen war, dass der Wasserstand des Sees durch die wasserwirtschaftlichen Maßnahmen bei durchschnittlichen Niederschlägen zwar weitgehend stabilisiert werden kann, eine Reihe trockener Jahre aber zu sehr niedrigen Seewasserständen bis hin zur Austrocknung führen kann. Die Wehranlage kann im Mittel höhere Pegel aufrechterhalten, aber letztendlich eine Austrocknung nicht verhindern.
Die Sorge um das potentielle Austrocknen des Neusiedlersees löste die Entwicklung von Konzepten zur Sicherung eines Mindestpegelstandes aus, weil Ökologie und Wirtschaft des Seewinkels in vielerlei Hinsicht an die Existenz des Neusiedlersees gebunden sind. Aus der spezifischen hydrologischen Situation des Neusiedlersees geht hervor, dass Austrocknung nur durch Zufuhr zusätzlichen Wassers zu verhindern wäre. Dafür käme Uferfiltrat von der Donau oder Wasser von der Raab, von ungarischer Seite, in Frage. Um den Seespiegel um $1 \mathrm{~mm}$ anzuheben ist allerdings eine Wassermenge von etwa $300000 \mathrm{~m}^{3}$ erforderlich (Donauuferfiltrat müsste auf ein $30 \mathrm{~m}$ höheres Niveau gepumpt werden - eine energieintensive Lösung). Die Raab hingegen hat gerade wenn der Neusiedlersee Wasserzufuhr bräuchte, selbst zumeist geringen Abfluss. Zusätzlich wurden ökologische Folgen der Zufuhr von Wasser mit anderer Nährstoffen und geringerer Salzfracht in den eher salzhaltigen Neusiedlersee diskutiert.

Selbst ein Absinken des Seepegels kann beträchtliche wirtschaftliche Auswirkungen haben (Schönerklee et al., 2008). Mit Hilfe eines digitalen Geländemodells des Neusiedlersees (Bácsatyai et al., 1997) wurde eine GIS Analyse der räumlichen Verteilung der Wassertiefen für unterschiedliche Wasserstände durchgeführt. Es wird ersichtlich, dass für Seepegel von 115,00 m Seehöhe ü.A. und 114,70 m Seehöhe ü.A. große Teile des Sees nur mehr sehr geringe Tiefen unter 1,2 m aufweisen.

Für Seepegel unter 115 m Seehöhe ü.A. errechneten Schönerklee et al. (2008) Einnahmenverluste von etwa 13 Mio $€$ und den Verlust von etwa 480 Arbeitsplätzen in der Region. Bei einem Absinken des Pegels um weitere $30 \mathrm{~cm}$, könnten die wirtschaftlichen Verluste das Dreifache erreichen und die Arbeitsplätze sich um das 2,5-fache reduzieren. Dies obwohl sich TouristInnen bei einer Befragung im Jahr 2006 nach der Methode des „Discrete Choice Experiments“ (kurz DCE) als eher unsensitiv gegenüber dem Wasserstand erwiesen (Pröbstl et al., 2007). Während Wochenendgäste in erster Linie die angebotenen Sportaktivitäten und Infrastruktur (Baden in See und Swimmingpool) als wichtig beurteilten, sind für UrlauberInnen vor allem die Landschaftsattribute des Wasserstandes und Naturerlebnisangebotes, sowie Kultur- und Weinerlebnis die wichtigsten Beurteilungskriterien. Die Wirkung geringerer Seepegel ist stark vom Landschaftstyp am Ufer (z. B. mit Schilfzone oder offenes Ufer, Schotterstrand) abhängig. NaturInteressierte zeigen deutlich stärkere Reaktionen auf die vorgelegten Bilder von hypothetischen Wasserstandsänderungen.

Anpassungsstrategien könnten die Diversifizierung des Tourismusangebotes und die zeitliche Erstreckung des Angebotes in den Frühling und Herbst, die weniger von Niedrigwasser bedroht sind, beinhalten. Jedenfalls wäre ein Maßnah- 
menbündel erforderlich, das neben dem Tourismus auch die Landwirtschaft und die Wasserwirtschaft umfasst (Schönerklee et al. 2008, Pröbstl et al., 2007).

\subsubsection{Die Bedeutung des Mittelmeeres für das Klima in Österreich}

Das Klima in Österreich ist wegen der geographischen Lage und dem Einfluss der Alpen sehr vielfältig. Hier treffen drei bedeutende Klimazonen aufeinander: Von Westen das feuchtmilde atlantisch geprägte Klima, von Osten das trockenere kontinentale Klima und von Süden das Mittelmeerklima. Zusätzlich verändern die Erhebungen der Alpen das Klima. So treten zum Beispiel Stau- und Abschattungseffekte des Niederschlags auf, regionale Wind-, Temperatur- und Feuchtefelder werden verändert, die sommerliche Gewittertätigkeit wird begünstigt und sogar Tiefdruckgebiete verändern unter dem Einfluss der Alpen ihre Zugbahn oder entstehen erst durch ihren Einfluss.

\section{Einfluss des Mittelmeeres}

Das Mittelmeer und in Bezug auf Österreich insbesondere die nahe gelegene Adria, kann Luft durch Verdunstung rasch mit Feuchtigkeit anreichern. Wird diese feuchte Luft in weiterer Folge in den Alpenraum transportiert, kommt es dort zu er-

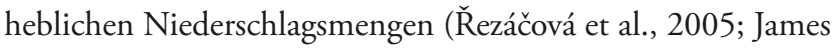
et al., 2004; Rudari et al., 2004; Zängl, 2004). Das ist insbesondere im Herbst im Süden Österreichs von Bedeutung, da über dem noch warmen Mittelmeer viel Wasser verdunstet und feuchte Luftmassen durch rege Tätigkeit von Tiefdruckgebieten häufig gegen die Alpen strömen. Südlich des 47. Breitengrads beträgt die Niederschlagssumme im Oktober daher durchschnittlich das 1,3 bis 1,6-fache des Mittelwerts aller Monate des Jahres. Auch für Neuschnee und damit für den Wintertourismus auf der Alpensüdseite ist das Mittelmeer von Relevanz, da hier Schneefälle im Spät-Herbst und Frühwinter oft durch Tiefdruckgebiete aus dem Mittelmeer ausgelöst werden.

Besonders wichtig sind Tiefdruckgebiete aus dem Mittelmeer für extreme Niederschlagsereignisse in Österreich. Dabei haben die Zugbahnen der Tiefdruckgebiete eine besondere Bedeutung (van Bebber 1882 und 1891; van Bebber und Köppen 1895). In seiner Arbeit benannte Van Bebber jene Tiefdrucksysteme, die von Genua ausgehend über Ostösterreich nach Polen ziehen, als „Vb“, jene die von Genua nach Osten ziehen als „Vc“ und solche die nach Südosten ziehen als „Vd“. Diese Tiefdrucksysteme können zu Hochwässern in Mitteleuropa führen (Mudelsee et al., 2004; Malitz und Schmidt, 1997). Viele verheerende Hochwässer der Vergangenheit wurden mit
Vb-artigen Zugbahnen in Verbindungen gebracht, so auch die Ereignisse im Juli 1997 (Kundzewicz et al., 2005), im August 2002 (z. B. Grazzini and Van der Grijn, 2002; Ulbrich et al., 2003; Rudolf und Rapp, 2003; Habersack und Moser, 2003) oder im August 2005 (z. B. Godina et al., 2006; Habersack und Krapesch, 2006).

Die Besonderheit dieser Wettersysteme liegt aber nicht nur im Transport feuchter Luftmassen in den Alpenraum, sondern auch in anderen Charakteristika begründet (Hofstätter und Böhm, 2011), wie z. B. in der geringen Verlagerungsgeschwindigkeit, der Langlebigkeit und der dynamischen Regeneration der Tiefdruckgebiete (Sekundärzyklogenese in Mesinger und Strickler 1982; Pichler und Steinacker, 1987) sowie in der orographischen und konvektiven Verstärkung der mit Wetterfronten verbundenen Niederschläge. Für Niederschlag im Alpenraum mit Herkunft der atmosphärischen Feuchte aus dem Mittelmeer stammt diese großteils aus dem westlichen Teil des Mittelmeeres. Der Feuchtebeitrag aus den Wasserflächen der nördlichen Adria hingegen ist auf die Gebiete von der PoEbene bis hin zum Alpenostrand beschränkt (Schicker et al., 2010). Dies unterstreicht die Bedeutung des Mittelmeeres für das Hochwasserrisiko in Mitteleuropa.

Die Analyse der oben beschriebenen Zugbahnen von Tiefdruckgebieten im Alpenraum in Kombination zeigt einen regional unterschiedlichen, aber deutlichen Zusammenhang mit Niederschlagsintensitäten (Hofstätter und Chimani, 2012). Für diese Analyse wurden die Zugbahnen aus der zeitlich-/räumlichen Verlagerung der Zentren von Tiefdruckgebieten mittels ERA-40 Daten bestimmt und großräumige (Gebietsdefinition nach Seibert et al., 2007) und voneinander unabhängige Niederschlagsereignisse aus dem Zeitraum 1963 bis 2002 betrachtet (Abbildung 5.10).

Für den westlichen Teil Österreichs (Tirol, Vorarlberg, Salzburg) zeigt sich dabei, dass besonders Zugbahnen des Typs Vc und $\mathrm{Vd}$ (dunkelrote Linie in Abbildung 5.10) für starke Niederschläge von Bedeutung sind. Im östlichen Teil Österreichs (Niederösterreich bis Oststeiermark) sind Vb-Zugbahnen (blaue Linien) von besonders hoher Relevanz für starke Niederschläge. Beim 98. Niederschlagsperzentil beträgt der Anteil von $\mathrm{Vb}$-Zugbahnen über $45 \%$.

Eine weitere Studie auf Basis der ERA40-Reanalyse (Awan und Formayer, 2012) stellt ebenfalls eine Verbindung zwischen großräumigen Starkniederschlägen und Vb-Tiefdruckgebieten her. Österreich zählt nach dieser Studie zu den am stärksten gefährdeten Regionen für extreme Niederschläge in Zusammenhang mit $\mathrm{Vb}$ Tiefdruckgebieten. Besonders relevant für Österreich sind dabei Tiefdruckgebiete die zentriert über den Alpen zu liegen kommen. 

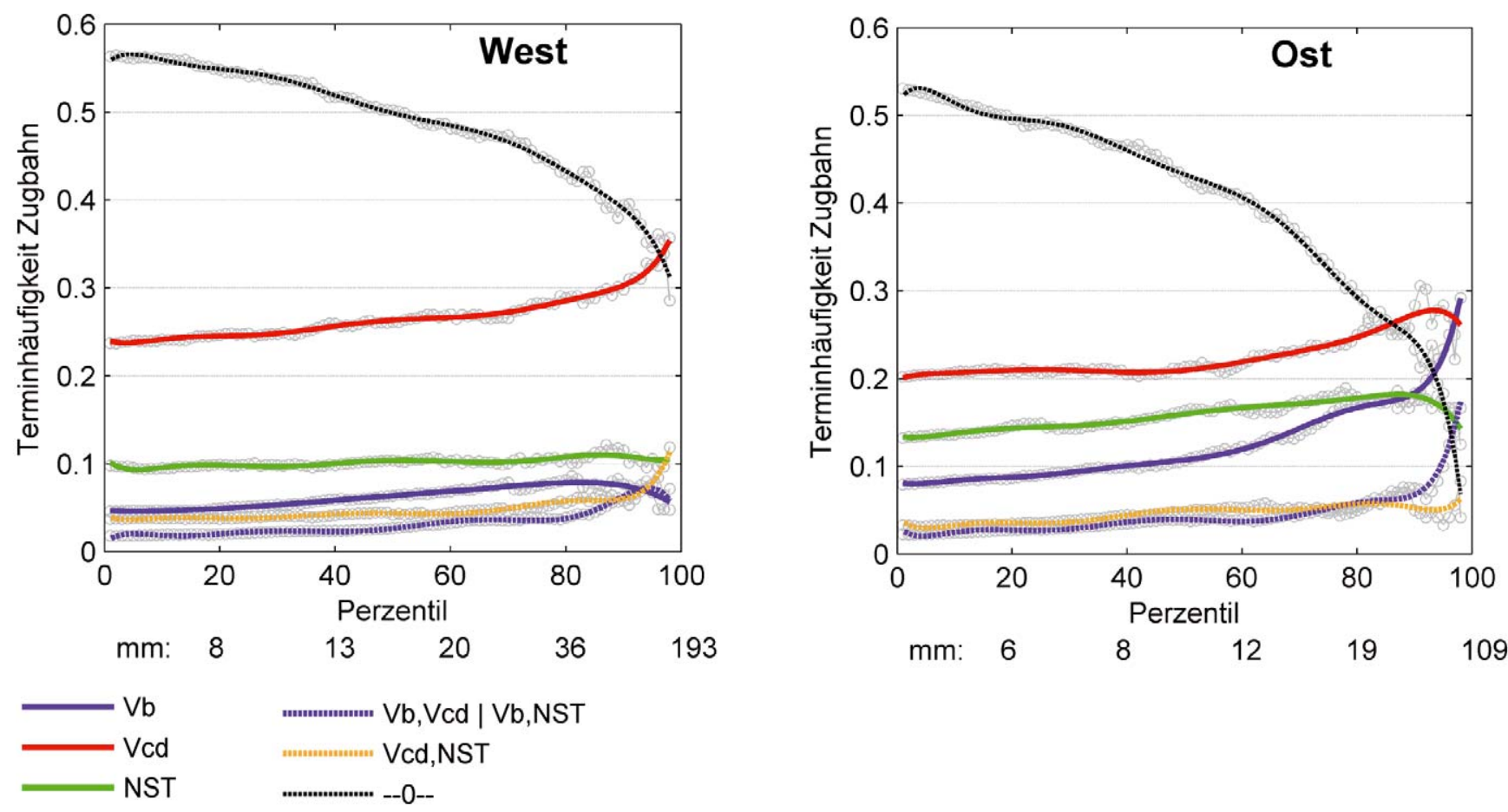

Abbildung 5.10 Relative Häufigkeit von verschiedenen Zugbahntypen bei Auswertung unabhängiger Niederschlagsereignisse für 1963 bis 2002 für die Gebiete "West" und "Ost" nach der Klassifikation von Seibert et al. (2007). Legende: Vb=Vb Zugbahn, Vcd=Vcd Zugbahn, NST=Tief über dem Alpenraum, $0=$ andere Typen. Quelle: Hofstätter und Chimani (2011)

Figure 5.10 Relative frequency of various track types „Zugbahnen” from the interpretation of independent precipitation events for the period 1963 to 2002 for the subregions "West" and "East" according to the classification of Seibert et al. (2007). Legend: Vb=Vb track, Vcd=Vcd track, NST=Low over the Alpine region, $0=$ other types. Source: Hofstätter und Chimani (2011)

Als weiterer Einfluss des Mittelmeeres auf das Wetter und Klima in Österreich ist die besonders gewitterträchtige Südwest-Wetterlage zu nennen, bei der warmfeuchte Luftmassen aus dem Bereich der Subtropen, auch unter Anfeuchtung über dem Mittelmeer, von Südwesten her nach Österreich geführt werden. Derartige Wetterlagen sind häufig mit Gewittern und konvektiven Starkniederschlägen verbunden, die in der Vergangenheit immer wieder zu lokalen Hangrutschungen und Vermurungen - wie beispielsweise im Juli 2012 - geführt haben. Auch das menschliche Wohlbefinden wird bei solchen Wetterlagen durch die schwüle Witterung beeinträchtigt.

\section{Klimazukunft}

Die oben beschriebene Vielfalt und Besonderheit der meteorologischen Phänomene sind nicht nur herausfordernd für die Messung und statistischen Beschreibung des Klimas, sondern bringen auch Klimamodelle rasch an die Grenzen ihrer Leistungsfähigkeit. Lange war zum Beispiel das Mittelmeer aufgrund von physikalisch-numerischen Beschränkungen und einer relativ groben räumlichen Auflösung nur unzureichend in den regionalen Klimamodellen abgebildet.

Seit etwa 2008 wird jedoch an der Entwicklung einer neuen Modellgeneration gearbeitet, bei der das Mittelmeer in seiner Gesamtheit in Klimasimulationen berücksichtigt wird (z. B. Sevault et al., 2009), sodass eine wesentlich besseren Abbildung der beobachteten Energie- und Masseflüsse zwischen Atmosphäre und dem Mittelmeer erzielt werden kann (Dubois et al., 2012). Simulationen mit diesen Modellen zeigen eine Reduktion des mittleren Niederschlags im Mittelmeerraum um 10-15\% in der Klimazukunft um 2050 (Emissionsszenario A1B). Wie sich die verbesserte Modellgüte auf die Abbildung von für Österreich relevanten Tiefdrucksystemen aus dem Mittelmeer und den dazu gehörigen Niederschlägen verhält, bleibt vorläufig noch offen.

Für das regionale Klimamodell Cosmo-CLM konnte gezeigt werden, dass Häufigkeit und Variabilität von Vb-Zugbahnen gut wiedergeben werden können (Hofstätter und Chimani, 2011). Weder für die Vergangenheit noch für die Zukunft (2030 bis 2070), konnte dabei eine signifikante Änderung der Häufigkeit von Vb-Zugbahnen gefunden werden. 
Tabelle 5.1 Schema der Wechselwirkung unterschiedlicher Prozesse und Fernwirkungsmechanismen welche direkt oder indirekt das Wetter und Klima im Alpenraum bestimmen. Abkürzungen: EA - Eastern Atlantic Pattern; ENSO - El Niño-Southern Oscillation; EP - Eastern Pacific Pattern; EU - Eastern European Pattern; NADW - North Atlantic Deep Water; NAO - North Atlantic Oscillation; NPO - North Pacific Oscillation; PNA - Pacific North American Pattern; QBO - Quasi-Biennal Oscillation; WA - West Atlantic Pattern; WP - West Pacific Pattern. Quelle: Wanner et al. (1997) übersetzt ins Deutsche

Table 5.1 Scheme of interactions between different processes and tele-conncetions affecting the weather and climate in the Alpine region either directly or indirectly. Abbreviations: EA - Eastern Atlantic Pattern; ENSO - El Niño-Southern Oscillation; EP - Eastern Pacific Pattern; EU - Eastern European Pattern; NADW - North Atlantic Deep Water; NAO - North Atlantic Oscillation; NPO - North Pacific Oscillation; PNA Pacific North American Pattern; QBO - Quasi-Biennal Oscillation; WA - West Atlantic Pattern; WP - West Pacific Pattern. Source: Wanner et al. (1997) translated into Geman

\begin{tabular}{|lll|}
\hline & Wichtige groß-skalige Prozesse und Fernwirkungsmechanismen & Räumliche Skala \\
\hline $\begin{array}{l}\text { ENSO } \\
\text { QBO }\end{array}$ & $\begin{array}{l}\text { Globaler Masse- und Energieaustausch: Ozeandynamik; Atmosphärisches } \\
\text { Wärme/Feuchte Budge; Globale Luftdruck- und Windfelder }\end{array}$ & Global \\
\hline Madden-Julian-Oszillation & $\begin{array}{l}\text { Dynamik der Hadley Zelle; Subtropischer Jetstream; Passatwinde; Subtropi- } \\
\text { scher Hochdruckgürtel }\end{array}$ & $\begin{array}{l}\text { Global bis hemisphä- } \\
\text { risch }\end{array}$ \\
\hline $\begin{array}{l}\text { NPO, PNA } \\
\text { EP, WP }\end{array}$ & $\begin{array}{l}\text { Ozeanische Zirkulation; Meeresoberflächentemperatur; Meereis; Atmosphäri- } \\
\text { sche Dynamik der mittleren Breiten }\end{array}$ & $\begin{array}{l}\text { Global bis hemisphä- } \\
\text { risch }\end{array}$ \\
\hline $\begin{array}{l}\text { NAO, WA } \\
\text { EA }\end{array}$ & $\begin{array}{l}\text { NADW Bildung; Dynamik des Nordatlantiks; Atmosphärische Zirkulation über } \\
\text { EU }\end{array}$ & $\begin{array}{l}\text { Hemisphärisch bis } \\
\text { Europa / Nordatlantik }\end{array}$ \\
\hline & Synoptisch-klimatologische Konfiguration über West- und Mitteleuropa & Kontinental (Europa) \\
\hline & Synoptisch-klimatologische Konfiguration: Wetter und Klima des Alpenraumes & Regional (Alpenraum) \\
\hline
\end{tabular}

\subsubsection{Veränderung großräumiger Zirkulations- muster ("Wetterlagen") im Klimawandel in Mitteleuropa und ihre Bedeutung für Österreich}

Unter einer Wetterlage wird der großräumige Zustand der Atmosphäre über einem bestimmten Gebiet verstanden. Zur Charakterisierung dieses Zustandes werden z. B. räumliche Muster des Luftdruckes, des Geopotentials oder der Temperatur verwendet. Eine detaillierte Beschreibung und ein Vergleich aktueller Klassifikationsmethoden ist in Philipp et al. (2010) zu finden. Diese wurden im Rahmen der COST Initiative 733 („Harmonisation and Applications of Weather Type Classifications for European Regions "1 , entwickelt, untersucht und für verschiedenen Anwendungen getestet. Kennt man die typischen Wettererscheinungen bei Vorherrschen einer bestimmten Wetterlage aus langjährigen Messungen, so kann aus dem jeweiligen Wetterlagentypus auf die regionale Witterung in einem Gebiet geschlossen werden. Die Prozesskette der atmosphärischen Zirkulation welche das regionale Wetter und Klima im Alpenraum bestimmt, wurde aus Wanner et al. (1997) entnommen und ist in Tabelle 5.1 dargestellt.

Eine langfristige Veränderung des globalen Klima kann zu Änderungen in der Häufigkeit, der Andauer oder auch in der Ausprägungsform von Wetterlagen führen Die Summe solcher

\footnotetext{
1 http://www.cost.eu/domains_actions/essem/Actions/733
}

Veränderungen führt - in Kombination mit überlagerten, natürlichen Variationen des Klimas - zu einer Modifikation der regionalen Witterung bzw. des Wetters über die jeweiligen Wetterlagen.

Ein Teil des über Europa beobachteten Temperaturanstieges (1850 bis 2003) lässt sich durch eine langfristige Veränderun der Häufigkeit bestimmter Wetterlagen erklären (Philipp et al., 2007). Ob die Veränderung der täglichen Zirkulationsmuster rein zufälliger Natur ist oder durch die globale Erwärmung ausgelöst wird, ist noch nicht hinreichend geklärt. Im 20. Jahrhundert dominierten in Mitteleuropa bis in die späten 1920er Jahre hinein zonale (Ost-West gerichtete) Zirkulationsformen. Danach zeigt sich bis Mitte der 1970er eine Zunahme der Häufigkeit von meridionalen Zirkulationsformen (Wanner et al., 1997). Bis zum Ende des 20. Jahrhunderts wurde eine stetige Zunahme des Luftdrucks im Alpenraums beobachtet, die sich durch eine deutliche Zunahme in der Andauer von Hochdruckgebieten über West- und Südwesteuropa erklären lässt.

Im Zeitraum 1979 bis 2002 finden Fricke und Kaminski (2002) eine ausgeprägte Zunahme von Südwest-Wetterlagen bei einer gleichzeitigen Abnahme von Nordwestwetterlagen in Mitteleuropa. Des Weiteren hat die Häufigkeit von zyklonalen Westlagen - solche bei denen ein Tiefdruckgebiet vom Atlantik her ostwärts über Europa zieht - vor allem im Sommerhalbjahr der Jahre 1881 bis 1998 abgenommen.

In einer anderen Arbeit (KLIWA Arbeitskreis, 2006) wurden für Süddeutschland meridionale-, zonale- und gemischte 
Zirkulationsformen hinsichtlich Langzeittrends untersucht. Im Winterhalbjahr nehmen dabei von 1950 bis 2000 zonale Zirkulationsformen deutlich ab, meridionale hingegen zu. Die Ergebnisse für das Sommerhalbjahr zeigen keine signifikanten Trends.

Zusammenfassend nimmt in Mitteleuropa bis dato der Einfluss meridionaler (süd-nord gerichteter) Zirkulationsformen zu, während der Einfluss von Wetterlagen die vom Atlantik her kommen zurückgeht. Unterschiede zwischen den verschiedenen Untersuchungen entstehen vor allem durch unterschiedliche Untersuchungsgebiete und unterschiedliche Methoden der Wetterlagenklassifikation.

Veränderungen der großräumigen Zirkulation in der Klimazukunft werden mit Hilfe von globalen Klimamodellen (GCMs) untersucht, doch konnten etwa in der Untersuchung von van Ulden und van Oldenborgh (2006), von 23 untersuchten GCMs lediglich acht Modelle die beobachtete Zirkulation und ihre Variabilität über Europa gut wiedergeben. $\mathrm{Zu}$ einem sehr ähnlichen Ergebnis kommen auch Casado und Pastor (2011). Die Modelle lassen jedenfalls eine Zunahme der Häufigkeit von Hochdruckgebieten über dem Westen und Südwesten Europas erwarten (siehe Abbildung 4.2 in Band 1, Kapitel 4). Das sommerliche Azorenhoch erstreckt sich demnach weiter nach Nordosten bis über Europa (z. B. Belleflamme, 2011).

\subsection{Die Entwicklung weiter denken: Überraschungen, abrupte Änderungen und Kipp-Punkte im Klimasystem}

\subsection{Thinking ahead: surprises, abrupt changes and tipping points in the climate system}

Allen bisherigen Überlegungen zum künftigen Klimawandel ist gemein, dass sie sich auf globale Klimamodelle und daraus abgeleitete Regionalisierungen stützen. Dabei darf nicht übersehen werden, dass Modelle Unsicherheiten unterschiedlicher Art unterliegen (vgl. Band 1, Kapitel 4), und keineswegs als getreues Abbild der Realität verstanden werden dürfen. Überraschungen sind daher durchaus möglich, ja sogar wahrscheinlich.

Unerwartete Wettersituationen und neue, überraschende Forschungsergebnisse helfen oft, bekannte und nicht erkannte Wissenslücken zu schließen. So wurden etwa in den 1980er Jahren in Europa Maßnahmen gegen die Versauerung der skandinavischen Seen und gegen das Waldsterben gesetzt. Dabei ging es vorwiegend um die Reduktion der Schwefeldioxid- emissionen und der dadurch verursachten atmosphärischen Sulfatbildung. Das Sulfat war als wesentliche Quelle der Versauerung der Seen und der Schädigung der Bäume erkannt worden. In den 1980er und 1990er Jahren wurde für Europa ein besonders starker Temperaturanstieg beobachtet, der deutlich über dem globalen Mittel lag (siehe Band 1, Kapitel 3). Dass Sulfataerosole die atmosphärischen Strahlungsflüsse beeinflussen war zwar bekannt, dass sie aufgrund der Erhöhung des atmosphärischen Sulfatgehaltes merkbar klimawirksam geworden waren, war jedoch ein überraschendes Ergebnis späterer Forschung (siehe z. B. Wild et al., 2005). Ergebnisse aus Strahlungsmessungen aber auch Modellexperimente zeigten deutlich zunächst den Effekt des sogenannten „Global Dimmings" und dann des „Global Brigthenings“. Die Reduktion der Sulfataerosolkonzentration wurde daher als zusätzlicher Antrieb (neben dem anthropogenen Treibhauseffekt) für den besonders starken Temperaturanstieg über Mitteleuropa seit ca. 1980 erkannt (Wild et al., 2005).

Eine aktuelle überraschende Entwicklung war die Aufstellung der Hypothese, dass die Auswirkungen des Klimawandels in der Arktis direkten Einfluss auf die Andauer, den Schneereichtum und das Temperaturniveau der Winter in Europa haben könnten (z. B. Petoukhov and Semenov, 2010, Jaiser et al., 2012). Reduzierte Meereisausdehnung im Sommer führt zu einem verstärkten Wärmeeintrag in die Atmosphäre und setzt die vertikale Stabilität der Atmosphäre herab. Dies könnte zu einer verstärkten Erzeugung atmosphärischen Wellen (planetare oder Rossby Wellen genannt) im Winter und damit einhergehend zu häufigeren Kaltluftvorstößen nach Europa führen (Jaiser et al., 2012). Eine andere Hypothese geht von vermehrtem Feuchtetransport in die Atmosphäre durch das fehlende Eis aus und von intensiviertem Blocking im Bereich der Arktis und dadurch verstärkten Kaltluftvorstößen Richtung Süden (Liu et al., 2012). Der Zusammenhang zwischen arktischer Meereisausdehnung und Winterniederschlag in Europa und Nordamerika ist jedenfalls nicht linear, so dass aus einem weiteren Rückgang des Meereises nicht direkt auf noch kältere und schneereichere Winter geschlossen werden kann.

Der Rückgang des Meereises in der Arktis ist auch ein Beispiel für eine sogenannte abrupte Änderung im Klimasystem: Die Eisbedeckung am Ende des Sommers ging einigermaßen linear mit dem Temperaturanstieg zurück, bis es im Jahr 2007 zu einer abrupten Abnahme kam, die weit über der von den Modellen erwarteten lag, obwohl die Modelle bis dahin gute Ergebnisse geliefert hatten (Stroeve et al., 2007). Der Rückgang der Eisbedeckung ist konsistent mit der Eis-Albedo-Rückkoppelung, die zur Erwärmung des Meeres und damit zum Schmelzen des Eises von unten beiträgt (Perovich et al., 2008). 
Das Schmelzen des Eises trägt wiederum zur Erwärmung der unteren Atmosphäre bei (Screen und Simmonds, 2012). Die daraus folgende Zunahme von Regen auf Kosten von Schneefall reduziert die Albedo des Eises (Screen und Simmonds, 2012). Auch Zirkulationsänderungen in den Ozeanen und in der Atmosphäre, sowie Änderungen in der Bewölkung trugen zu der Abnahme der Eisausdehnung bei (Lenton, 2012).

Derartige abrupte Änderungen können auch in anderen Elementen des Klimasystems vorkommen. Während manche Prozesse im Klimasystem eher linearen Charakter aufweisen, sind andere in hohem Maße nicht-linear, sodass Auswirkungen einer Änderung über- oder unterproportional gegenüber der Änderung ausfallen können. Änderungen werden als abrupt definiert, wenn eine großräumige Änderung im Klimasystem sich innerhalb einiger Dekaden oder weniger einstellt, und mindestens über einige Dekaden anhält und wesentliche Störungen in natürlichen oder menschengemachten Systemen auslöst (IPCC, 2013). Derartige Störungen sind an sich schwer zu erfassen und ihr Eintreten schwer vorhersagbar, doch ist bekannt, dass verschiedene Komponenten oder Phänomene des Klimasystems potentiell abrupten Änderungen unterliegen könnten und in der Vergangenheit unterlegen sind (Dakos et al., 2008; Scheffer et al., 2009). Beispiele wären neben dem bereits erwähnten Arktischen Meereis, das Grönland Eisschild, die Atlantic Meridional Overturning Circulation, der Amazonas Regenwald und Monsunzirkulationen. Obwohl für einige dieser Systeme bereits Analysen hinsichtlich möglicher Konsequenzen durchgeführt wurden, bleiben die Ergebnisse unsicher und unter WissenschafterInnen umstritten (IPCC, 2013).

Eng verknüpft mit dieser Thematik ist die Frage der Irreversibilität der Änderungen, d.h. ob die Änderungen in einem Zeitraum rückgängig gemacht werden können, der vergleichbar mit dem Zeitraum der Entwicklung der Änderung ist (IPCC, 2013). Wegen der langen Lebensdauer des $\mathrm{CO}_{2}$ in der Atmosphäre muss in diesem Kontext der Klimawandel an sich als irreversibel bezeichnet werden (Solomon et al., 2009).

Von besonderer Bedeutung für die Dringlichkeit von Klimaschutzmaßnahmen ist die Frage der sogenannten KippPunkte, die mit Irreversibilität einhergehen können. Durch positive Rückkoppelungen im Klimasystem können angestoßene Veränderungen sich verstärken. Die Eis-Albedo-Rückkoppelung und die Treibhausgasrückkoppelung wurden bereits erwähnt (vgl. Band 1, Kapitel 1). Sie können dazu führen, dass eine Entwicklung zur Erwärmung oder zur Abkühlung, sobald einmal losgetreten, sich von selber, ohne dass der Auslöser weiterhin aktiv sein muss, fortsetzt, ja unter Umständen sogar beschleunigt. Wenn dies im Klimasystem eintritt, spricht man von der Überschreitung von Kipp-Punkten (Lenton et al., 2008; Hansen et al., 2008; Hansen 2009).

Im IPCC, 2013 wird angemerkt, dass nur wenige, auf vereinfachenden Modellen beruhende Studien Belege für globale Kipp-Punkte finden (z. B. Lenton et al., 2008), während Studien mit umfassenderen Klimamodellen innerhalb des 21. Jahrhunderts keine Hinweise auf Kipp-Punkte liefern. Die Ozeanzirkulation und Eisschilde könnten im Maßstab von einigen Jahrhunderten bis zu Jahrtausenden solche aufweisen, regional eventuell auch die Arktis (Lenton, 2012; Wadhams, 2012), obwohl die Befunde hier auseinandergehen (Armour et al., 2011; Tietsche et al., 2011). Ein weiteres System, das Kipp-Elemente (Tipping Elements TE nach Lenton et al., 2008) enthalten könnten, sind das El Niño/Southern Oscillation (ENSO-) System, dessen Kipp-Punkte zu erforschen die gegenwärtigen Klimamodelle nicht in der Lage sind (Latif und Keenlyside, 2009).

Es sind dies typische Beispiele für unsymmetrische, schiefe Verteilungen mit einem „dicken Ende“ (fat tail): Trägt man den verursachten Schaden gegen die Wahrscheinlichkeit des Auftretens eines Ereignisses auf, dann kann der Schaden etwas kleiner oder etwas größer sein als beim wahrscheinlichsten Ereignis, er kann auch viel ärger sein, aber nicht viel geringer (National Research Council, 2013). Im Sinne der Umweltethik sind in einer derartigen Situation auch die viel schlimmeren Konsequenzen zu diskutieren (siehe Abschnitt 5.2). In den letzten Jahren ist es gelungen, für einige der Extreme ein gewisses $\mathrm{Ma}$ an Entwarnung zu geben: so gilt es jetzt als viel unwahrscheinlicher, dass es zu einer Verlagerung des Abtauchens der Thermohalinen Zirkulation in den mittleren Atlantik und damit zu einer plötzlichen, signifikanten Abkühlung in Europa kommt (National Research Council, 2013). Auch die unkontrollierte Freisetzung größerer Mengen von Methan aus den Ozeanen gilt inzwischen als wenig wahrscheinlich (National Research Council, 2013). In den meisten Fällen kann jedoch die Frage nach Kipp-Punkten weder eindeutig verneint, noch eindeutig bejaht werden (Schellnhuber, 2009). Großskalige Störungen (disruptive changes) im globalen System sind in der Regel nicht in Modellexperimenten enthalten und selten in Wirkungsanalysen. Mit Annäherung und Überschreiten der $2^{\circ} \mathrm{C}$ Grenze wird das Überschreiten von KippPunkten und damit das Auftreten abrupter Änderungen wahrscheinlicher (PIK, 2012).

Die National Academy of Science der USA widmete den Kipp-Punkten 2013 einen eigenen Bericht, in dem u. a. vorgeschlagen wird, ein Monitoring System einzurichten, um frühe Anzeichen von abrupten Änderungen und Kipp-Punkten zu erkennen (National Research Council, 2013). In dieser Studie 
werden nicht nur Kipp-Punkte im Klimasystem untersucht, sondern auch „Wirkungs-Kipp-Punkte“, d.h. solche in den vom Klimawandel betroffenen natürlichen Systemen. Schellnhuber (2009) wies schon darauf hin, dass auch ökonomische, soziale und politische Systeme Kipp-Punkte aufweisen könnten, die als Folge des Klimawandels überschritten werden könnten.

Untersuchungen über die potentiellen Auswirkungen abrupter Änderungen oder des Überschreitens von KippPunkten im globalen oder regionalen Maßstab auf das Klima in Österreich liegen nicht vor. Für Österreich könnten aber derartige Vorgänge in kleinerem Maßstab von Bedeutung sein: etwa hinsichtlich des Rückzuges von Gletschern oder der Stabilität von Gebirgshängen. Auch hier sind entsprechende Studien noch ausständig.

\subsection{Forschungsbedarf \\ 5.6 Research needs}

Der aktuelle Forschungsstand zum Klimawandel in Österreich wurde in den Kapiteln 2 bis 4 eingehend dargestellt. Es wurde auch aufgezeigt, welchen konkreten Forschungsfragen nachzugehen ist, um das Verständnis über den Klimawandel zu verbessern und um gezielt Maßnahmen zur Anpassung oder Emissionsminderung setzen zu können. Hier wird dieser Forschungsbedarf nochmals im Gesamtkontext zusammengefasst.

Die Voraussetzung für das Verständnis des Klimawandels und des Einflusses des Menschen auf das Klima ist einerseits die fortlaufende, langfristige Messung wesentlicher Klimakenngrößen sowie deren Analyse. Andererseits ist es notwendig die atmosphärischen Vorgänge und deren Wechselwirkungen, auch mit den anderen Erdsphären, modellhaft abzubilden.

Klimabeobachtungen haben in Österreich eine lange Tradition, während die Beobachtungsreihen für THG und Aerosole wesentlich jünger und weniger zahlreich sind. Folglich fehlen wichtige Informationen für die Ermittlung der natürlichen THG-Emissionen in Österreich. Systematische, kontinuierliche Aerosolbeobachtungen stehen erst am Anfang. Dies gilt nicht nur für Österreich, sondern auch auf internationaler Ebene. Dementsprechend ist auch das Wissen über THG, deren Verhalten in der Atmosphäre und ihr Einfluss auf den Strahlungshaushalt weiter fortgeschritten, während die vielfältigen, und räumlich variableren Prozesse der Aerosolbildung und -wirkung wesentlich weniger gut bekannt sind.

Mit dem Hohen Sonnblick hat Österreich eine hervorragende Hintergrundstation für atmosphärenchemische Mes- sungen. Neben den sehr wertvollen, mehr als 125 Jahre umfassenden, Zeitreihen der meteorologischen Standardgrößen existieren mittlerweile zum Beispiel auch lange, praktisch unterbrechungslose Zeitreihen von stratosphärischem Ozon und UV-Strahlung am Sonnblick. Mögliche Auswirkungen des Klimawandels auf die Stratosphäre und die Erholung der Ozonschicht werden heute nur teilweise verstanden, weitere Forschungsarbeiten sind erforderlich, um bessere Aussagen über die zukünftige Entwicklung der Ozonschicht, die auch Einfluss auf die globale Strahlungsbilanz und damit das Klima ausübt, treffen zu können. Die Erweiterung der Strahlungsmessungen, die Teil des Baseline Surface Radiation Networks (BSRN) sind, durch Messungen der spektralen Albedo wäre ein wichtiger Schritt zum besseren Verständnis von Rückkoppelungsprozessen der Strahlungsbilanz und eine wichtige Schnittstelle zu Satellitenanwendungen. Neben dem Sonnblick wären jedoch auch zusätzliche Hintergrundmessstellen in Österreich für eine bessere räumliche Erfassung der atmosphärischen Prozesse wichtig.

Methoden der inversen Modellierung können Emissionsdaten validieren und so Verbesserungen für Inventuren ermöglichen. Österreich hat zur Entwicklung dieser relativ jungen Modellierverfahren beigetragen, doch könnten diese durch vermehrte Forschung noch wesentlich verbessert werden.

Zum Verständnis aktueller und künftiger Klimaprozesse kann die Kenntnis vergangener Entwicklungen Wesentliches beitragen. Ein zentrales Anliegen der Paläoklimatologie ist die Ausweitung der Proxyinformationen auf weitere Klimaelemente und auf andere Jahreszeiten (derzeit liegen vorwiegend Sommerinformationen vor). Mit verbesserten Chronologien und mehr Informationen über vergangene Extremereignisse, vor allem im Holozän, könnte die natürliche Variabilität auf sehr kurzen Zeitskalen quantitativ besser eingeschätzt werden. Auch die Untersuchung der „abrupten Klimaänderungen“ im Pleistozän verspricht das Prozessverständnis von Klimaänderungen auf regionaler und lokaler Ebene zu verbessern. Hohes Potential zur zeitlichen und räumlichen Verdichtung der Klimainformation in Österreich bieten zahlreiche, noch nicht ausgewertete historische Dokumente - ein international weit verbreiteter und ergiebiger Forschungsansatz.

Das an sich gute meteorologische Datenmaterial erfordert hinsichtlich Datenqualität und Datendichte weitere Verbesserungen. Fortsetzung und Ausweitung der Digitalisierung und Homogenisierung instrumenteller Zeitreihen im Hinblick auf zusätzliche Klimaparameter und Ausbau in täglicher bzw. subtäglicher Auflösung sind zwingende Voraussetzung für ein besseres Verständnis der Veränderungen von Extremwerten. Geeignete Methoden zur räumlichen Interpolation der $\mathrm{Da}-$ 
ten in komplexer Topographie sind zu entwickeln, auch unter Verwendung jüngerer Datensätze wie etwa Radar- oder Blitzortungsdaten und indirekt nutzbarer Daten aus Klimafolgen. Hier ist interdisziplinäre Kooperation notwendig. Durch eine internationale Kooperation zur Erstellung eines langjährigen und räumlich hochauflösenden Reanalysedatensatzes für den Alpenraum könnte eine einzigartige Grundlage für die Modellverbesserung von regionalen Klimamodellen und für die Modellierung von Klimafolgen erstellt werden.

Das kürzlich in Österreich initiierte hochqualitative Strahlungsmonitoring (ARAD Messnetz) würde durch eine Ausweitung auf die gesamte Energiebilanz wesentlich gewinnen. Insbesondere der latente Wärmefluss bzw. die damit zusammenhängende Verdunstung sind noch viel zu wenig bekannte Größen der Energie- bzw. Wasserbilanz, die daher auch in meteorologischen und hydrologischen Modellen nicht gut genug abgebildet werden können.

Für viele für den Menschen relevante Wetterereignisse, wie etwa Hochwasser oder Lawinen, ist es vorteilhaft meteorologische Abläufe über einige Tage zu erfassen, da sich meteorologische Ereignisse oft in ähnlicher Weise entwickeln und wiederholen. Die synoptische Klimatologie verfolgt einen dafür geeigneten Ansatz auf der Skala der Wetterphänomene (z. B. Hoch-, Tiefdruckgebiete, Fronten) bei gleichzeitiger Berücksichtigung von kleinräumigen meteorologischen Phänomenen. Die Klassifizierung der atmosphärischen Zirkulation in dominante Muster und Typen ermöglicht es die zugrunde liegenden Prozesse genauer zu berücksichtigen. Dadurch können dann schärfere Aussagen u.a. für Extremwert- und Variabilitätsanalysen, auch unter zukünftigen Klimazuständen, abgeleitet werden. Hier liegen erst wenige Arbeiten für Österreich vor.

Basis für alle Simulationen des zukünftigen Klimas sind globale Klimamodelle. Österreichische ForscherInnen greifen auf die international verfügbaren Modellergebnisse zurück, da globale Klimamodelle in Österreich nicht gerechnet werden. Ein wichtiger Schritt vor Nutzung der Ergebnisse ist die Validierung der Modelle für Österreich, insbesondere im alpinen Raum. Fehler, die in den Globalen Klimamodellen enthalten sind, lassen sich durch regionale Modelle nicht beheben. Die Entwicklung von Validierungsverfahren, die den globalen Modellen, den verfügbaren Daten und der geplanten weiteren Nutzung angemessen sind, ist eine meist unbedankte, aber wichtige Forschungsaufgabe. Eine Zusammenarbeit zwischen den EntwicklerInnen und NutzerInnen von Regionalmodellen und den EntwicklerInnen von Globalmodellen wäre wichtig, damit die Probleme im Alpenraum bzw. in Gebirgen vermehrt auch bei der Entwicklung von Globalmodellen wahrgenommen werden.
Derzeit stellen empirisch-statistische Verfahren eine wichtige Schnittstelle zwischen den Ergebnissen der Klimamodelle und den Eingangsdaten für die Simulation von Klimafolgen dar. Statistische Regionalisierung, Fehlerkorrektur oder die Abschätzung von Unsicherheiten sind wichtige Ziele der empirisch-statistischen Verfahren. Diese Methoden sind teilweise noch sehr rudimentär und haben deutliches Verbesserungspotential. Stärkere Förderung in diesem Bereich könnte die Qualität von Studien zur den Auswirkungen des Klimawandels, durch hohe Effektivität der Methoden, in den kommenden Jahren deutlich verbessern. Empirisch-statistische Verfahren können jedoch physikalische Modelle nicht ersetzen sondern nur ergänzen.

Es muss daher langfristiges Ziel sein, die Qualität der dynamischen Klimamodelle zu verbessern, damit im Idealfall eine Fehlerkorrektur mittels empirisch-statistischer Verfahren obsolet wird. Nur in den dynamischen Modellen werden Prozesse und Rückkopplungseffekte direkt erfasst und können daher auch in die Zukunft projiziert werden. Bei der in Österreich sehr regen regionalen Klimamodellierung geht es speziell um eine bessere Abbildung jener Prozesse, die im Alpenraum relevant sind. Neben einer weiteren Verfeinerung der räumlichen Auflösung muss auch an der Integration weiterer Prozesse gearbeitet werden. Wesentliche Ansatzpunkte sind: Modellparametrisierungen (z. B. Konvektion, Wolkenbildung, Schneeschmelze, Mikrophysik, Planetare Grenzschicht oder Turbulenz), Erhaltungsgrößen und physikalische Konsistenz; numerische Probleme im steilen Gelände; Probleme bei der Erhöhung der räumlich-zeitlichen Auflösung.

Ein offenkundiges Defizit der regionalen Modellierung ist die Bereitstellung jener Informationen, die in Klimafolgenuntersuchungen gebraucht werden. Systematische Untersuchung des Bedarfes und Abstimmung desselben mit ermittelbaren Kenngrößen könnte wesentliche Fortschritte bei der Folgenuntersuchung bewirken. Auch die Genauigkeitsanforderungen der Folgenforschung wären zu präzisieren, um auch weniger gut abgesicherte meteorologische Größen, wie Niederschlag, Feuchte, Wind, etc. besser nutzen zu können.

Das Verständnis für die Bedeutung der Schnittstelle zwischen meteorologisch-klimatologischen Fragestellungen und Fragen der Auswirkungen des Klimawandels auf natürliche und anthropogene Systeme muss gestärkt werden, um die Kompatibilität zwischen klimatologischen Ergebnissen und den Erfordernissen an Eingangsdaten für die Folgenforschung zu gewährleisten. Methodische Fortschritte zur Verbesserung dieser Schnittstellen sind erforderlich. Dabei geht es um Verbesserungen beim Implementieren von Sub-Modellen und Modellkopplungen und zwar nicht so sehr um die rein nu- 
merische Ankoppelung, sondern um sinnvolle Verknüpfungen auf Basis des Verständnisses der Leistungsfähigkeit und der Erfordernisse der zu koppelnden Modelle. Beispiele sind die Koppelung regionaler Klimamodelle mit hydrologischen Modellen oder dynamischen Vegetationsmodellen oder das Einbauen biogeochemischer Zyklen. Von besonderer Bedeutung ist die vertiefte Analyse der Unsicherheiten und deren Auswirkungen auf die Folgenforschung.

Viele kleinräumige Phänomene werden derzeit weder messtechnisch noch durch regionale Klimamodelle zufriedenstellend erfasst. Es ist kein Zufall, dass sich zu kleinräumigen Extremereignissen, wie Gewitter, Hagel, Tornados oder Starkniederschlägen auch im vorliegenden Band des AAR14 wenig Information findet. Klimafolgenuntersuchungen, insbesondere ökonomische Analysen, erfordern jedoch vor allem Angaben über Häufigkeit und Intensität extremer wetterbedingter Ereignisse. Methodische Weiterentwicklungen und alternative Ansätze sind nötig, um Aussagen über klimabedingte Veränderungen kleinräumiger Prozesse und deren Auswirkungen machen zu können.

Angesichts der Tatsache dass ein wesentlicher Teil der österreichischen Bevölkerung in Städten lebt, stellt die Untersuchung der zu erwartenden Klimaveränderungen von städtischen Gebieten eine wesentliche Herausforderung der Zukunft dar. Für die Bundeshauptstadt Wien besteht sowohl hinsichtlich Datenerhebung als auch Modellierung ein Wissensdefizit. Ein Vergleich auf internationaler Ebene zeigt, dass andere Städte wesentlich besser untersucht sind. Die Forschung zur Entwicklung der Stadt Wien im Klimawandel läuft erst jetzt an und muss noch deutlich verstärkt werden, insbesondere im Blickpunkt des für die nächsten Jahre prognostizierten Zuzugs nach Wien und der fortschreitenden Urbanisierung des Umlandes. Besonderes Augenmerk sollte in Österreich auch Städten mit 20- bis 50000 EinwohnerInnen geschenkt werden. Städte dieser Größe weisen schon städtisches Mikroklima auf, sehen sich aber finanziell in der Regel nicht in der Lage, Messnetze zu betreiben oder Klimastudien durchführen zu lassen.

Schließlich fehlen für Österreich Analysen über Auswirkungen globaler abrupter Änderungen oder Kipp-Punkte. Die Analyse der Auswirkungen des Verlustes von arktischem Polareis für Österreich läuft derzeit erst zaghaft an. Diese Art der Forschung zeigt deutlich, dass sich Österreich an globalen Forschungsaufgaben wie etwa der Polarforschung nicht nur aus Gründen der globalen Verantwortung sondern auch aus Gründen der nationalen Auswirkungen beteiligen muss. Zu möglichen Auswirkungen globaler abrupter Änderungen auf Österreich und zu lokalen abrupten Änderungen liegen noch keine Studien vor.
Abschließend sei noch angemerkt, dass auch Forschung zur Kommunikation des Klimawandels, insbesondere ethischer, psychologischer und soziologischer Aspekte für die KlimaforscherInnen Österreichs von Bedeutung wäre.

\subsection{Aussagen zum Klimawandel und deren Belastbarkeit \\ 5.7 Statements on climate change and their resilience}

Im folgenden Abschnitt wird eine Auswahl der in den Kapiteln 1 bis 4 dargestellten Befunde zum Klimawandel global (Tabelle 5.2) und in Österreich (Tabelle 5.3) dargestellt. Damit soll nochmals in Kurzform der Stand des Wissens zu einzelnen Themenbereichen des Klimawandels vor allem aber auch die entsprechende Unsicherheit aufgezeigt werden. Bei der Bewertung der Unsicherheit wird der Grad der Sicherheit ( $S$, aus der Qualität und den Umfang der Datenbasis abgeschätzt) einerseits und der Grad der Übereinstimmung unter WissenschafterInnen (Ü) andererseits, im Sinne der Unsicherheitsskala des IPCC (2013) Berichtes angegeben. 
Tabelle 5.2 Wichtige Aussagen zur Klimaänderung auf globaler Skala mit hoher Relevanz für den Alpenraum und in Österreich. S beschreibt Qualität und den Umfang der Datenbasis, Ü den Grad der Übereinstimmung unter Wissenschafterlnnen [H = hoch, M= mittel, N= niedrig] Table 5.2 Important statements on the climate change at the global scale with high relevance for the Alpine region and Austria. $\mathrm{S}$ describes

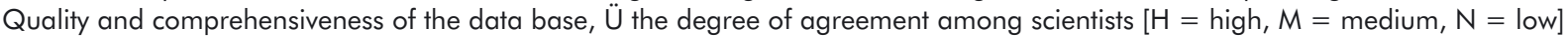

\begin{tabular}{|c|c|c|c|c|}
\hline \multicolumn{2}{|c|}{ Aussage } & \multirow[t]{2}{*}{ S } & \multirow{2}{*}{ Ü } & \multirow{2}{*}{$\begin{array}{l}\text { AAR14 } \\
\text { Kapitel } \\
1 / 1\end{array}$} \\
\hline 1 & $\begin{array}{l}\text { Das globale Temperaturmittel } 2003 \text { bis } 2012 \text { war um } 0,78^{\circ} \mathrm{C} \text { (mit } 90 \% \text { iger Sicherheit zwischen } 0,72-0,85^{\circ} \mathrm{C} \text { ) } \\
\text { wärmer als das Mittel } 1850 \text { bis } 1900 \text { (IPCC, 2013). Den Temperaturanstieg zeigen meteorologische Messungen } \\
\text { ebenso wie qualitative Beobachtungen der belebten Natur. }\end{array}$ & & & \\
\hline 2 & $\begin{array}{l}\text { Änderungen des Klimas sind an sich nichts Ungewöhnliches: Der stete Wechsel von langen Glazialen und kurzen } \\
\text { Interglazialen ist das prägende Bild des globalen Klimas im Quartär. Die präzise bekannten, langfristigen Schwan- } \\
\text { kungen der Erdbahnelemente bewirken eine Änderung der Sonnenenergie welche über Rückkoppelungen Eiszeit- } \\
\text { Warmzeit-Zyklen stevert (Milankovitch-Theorie). }\end{array}$ & $\mathrm{H}$ & $\mathrm{H}$ & $\begin{array}{l}1 / 1 \\
\text { und } \\
1 / 3\end{array}$ \\
\hline 3 & $\begin{array}{l}\text { Für die langfristige Zukunft ist prinzipiell mit weiteren Eiszeit-Warmzeit-Zyklen zu rechnen. Die Dauer des gegen- } \\
\text { wärtigen Interglazials, des Holozäns, ist jedoch unklar, da nach Modellrechnungen beim derzeitigen Niveau des } \\
\mathrm{CO}_{2} \text {-Gehalts der Atmosphäre (rund } 390 \text { ppm) keine neuerliche Vereisung eintreten wird. }\end{array}$ & $\mathrm{H}$ & $\mathrm{H}$ & $\begin{array}{l}1 / 1 \\
\text { und } \\
1 / 3\end{array}$ \\
\hline 4 & $\begin{array}{l}\text { Wechselwirkungen zwischen Atmosphäre und Ozean sind eine wesentliche Ursache für Klimaschwankungen auf } \\
\text { der Zeitskala von Jahren bis Jahrhunderten. Für das europäische Klima hoch relevant ist die atlantische meridio- } \\
\text { nale Tiefenzirkulation (AMOC), von der ein wesentlicher Teil der Nordatlantikstrom („Golfstrom“) ist. Damit gekop- } \\
\text { pelt ist die nordatlantische Oszillation (NAO), die mit Klimaschwankungen in Europa vor allem im Winterhalbjahr } \\
\text { in Verbindung steht. }\end{array}$ & $\mathrm{H}$ & $M$ & $1 / 1$ \\
\hline 5 & $\begin{array}{l}\text { Eine Erhöhung der natürlichen THG-Konzentrationen in der Atmosphäre führt zu einer Verstärkung des Treibhaus- } \\
\text { effektes und damit zur Erwärmung, da die derzeit herrschenden THG-Konzentrationen nur einen sehr kleinen Teil } \\
\text { der Ausstrahlung der Erde absorbieren. „Sättigung” ist noch für kein THG erreicht. }\end{array}$ & $\mathrm{H}$ & $M$ & $1 / 1$ \\
\hline 6 & $\begin{array}{l}\text { Wasserdampf ist jenes Gas, das die stärkste direkte Treibhauswirkung besitzt. Seine Konzentration steigt u.a. infol- } \\
\text { ge erhöhter Verdunstung von den Ozeanen mit der globalen Erwärmung. }{ }^{1}\end{array}$ & $\mathrm{H}$ & $\mathrm{H}$ & $1 / 1$ \\
\hline 7 & $\begin{array}{l}\text { Der Anstieg der Kohlendioxidkonzentration in der Atmosphäre ist auf anthropogene Quellen (vorwiegend Verbren- } \\
\text { nung fossiler Brennstoffe) zurückzuführen; er wird gedämpft durch erhöhte Kohlenstoffaufnahme der Ozeane und } \\
\text { der Biosphäre. }\end{array}$ & $\mathrm{H}$ & $\mathrm{H}$ & $\begin{array}{l}1 / 1 \\
\text { und } \\
1 / 2\end{array}$ \\
\hline 8 & $\begin{array}{l}\text { Die in den letzten Jahrzehnten beobachteten Temperaturänderungen sind nach heutigem Verständnis des } \\
\text { Klimasystems nur zu erklären, wenn der Einfluss der anthropogenen THG-Emissionen berücksichtigt wird. }\end{array}$ & $\mathrm{H}$ & $\mathrm{H}$ & $1 / 1$ \\
\hline 9 & $\begin{array}{l}\text { Der Beitrag von Intensitätsänderungen der Sonnenstrahlung zur beobachteten Erwärmung liegt bei ca. einem } \\
\text { Zwanzigstel jenes der THGe (ohne Wasserdampf). Jedoch ist das wissenschaftliche Verständnis über die Wirkung } \\
\text { von THG viel besser als das über die Änderungen der Solarstrahlung. }\end{array}$ & $\mathrm{H}$ & $\mathrm{H}$ & $1 / 1$ \\
\hline 10 & $\begin{array}{l}\text { Die relativ geringe Erwärmung im letzten Jahrzehnt ist wahrscheinlich zu einem Großteil auf Klimavariabilität } \\
\text { zurückzuführen, insbesondere auf eine episodische Speicherung großer Energiemengen in den Ozeanen. }\end{array}$ & $M$ & $M$ & $1 / 1$ \\
\hline 11 & $\begin{array}{l}\text { Kleine Ursachen können im komplexen, nichtlinearen Klimasystem große Wirkungen haben, wenn Gleichgewichte } \\
\text { gestört und Rückkoppelungsmechanismen ausgelöst werden. Der Mensch kann deshalb merkbar in das Klimasys- } \\
\text { tem eingreifen. }\end{array}$ & $\mathrm{H}$ & $\mathrm{H}$ & $1 / 1$ \\
\hline 12 & $\begin{array}{l}\text { Szenarienberechnungen für die Zukunft ergeben - je nach Entwicklung von Bevölkerungszahlen, Wirtschaft, } \\
\text { Technologie, etc. ohne Berücksichtigung der Unsicherheiten - eine globale Temperaturerhöhung zwischen } 2^{\circ} \mathrm{C} \\
(\mathrm{RCP} 2.6) \text { und } 3,5^{\circ} \mathrm{C} \text { (RCP8.5) bis } 2100 \text { gegenüber heute. }{ }^{2}\end{array}$ & $M$ & $M$ & $1 / 1$ \\
\hline 13 & $\begin{array}{l}\text { Zusammen mit Verschiebungen in den mittleren Bedingungen verändern sich auch Häufigkeit und Intensität extre- } \\
\text { mer Ereignisse. }\end{array}$ & $M$ & $\mathrm{H}$ & $1 / 1$ \\
\hline 14 & $\begin{array}{l}\text { Bereits beobachtete Zunahmen der Häufigkeiten oder Intensitäten extremer Wetterereignisse können, müssen aber } \\
\text { nicht, mit dem Klimawandel zusammenhängen. }\end{array}$ & $\mathrm{H}$ & $\mathrm{H}$ & $1 / 1$ \\
\hline 15 & $\begin{array}{l}\text { Das international akkordierte politische Ziel von maximal } 2^{\circ} \mathrm{C} \text { Erwärmung gegenüber dem vorindustriellen Tempe- } \\
\text { raturniveau wird nur im ehrgeizigsten Konzentrationspfad (RC2.6 nach IPCC, 2013) erreicht. Größere Unterschie- } \\
\text { de zwischen einzelnen Szenarien treten erst Mitte des } 21 \text {. Jahrhunderts auf. }\end{array}$ & $\mathrm{N}$ & $M$ & $1 / 1$ \\
\hline 16 & $\begin{array}{l}\text { Aus Modellsimulationen ist abzuleiten, dass in Nord- und Zentraleuropa Niederschlagsextreme eher an Intensität } \\
\text { und Häufigkeit zunehmen, für den Mittelmeerraum ist dagegen eine Änderung von Extremniederschlägen unge- } \\
\text { wiss. }\end{array}$ & $M$ & $M$ & $1 / 4$ \\
\hline
\end{tabular}

1 Änderungen in der Wasserdampfkonzentration werden den Auswirkungen des Klimawandels zugerechnet, nicht den anthropogenen THG-Emissionen. Wasserdampf ist jenes Gas, das die stärkste direkte Treibhauswirkung entfaltet. Aufgrund seiner kurzen atmosphärischen Lebenszeit ist seine Konzentration primär von der Temperatur bestimmt, die die Verdunstung aus Ozeanen beeinflusst; es wirkt somit vor allem als Verstärker der Effekte anderer, langlebiger THGe.

2 Diese Berechnungen setzen im sozio-ökonomischen Bereich Kontinuität voraus. Brüche sind nicht berücksichtigt. Wichtige Aussagen zur Klimaänderung im Alpenraum und in Österreich basierend auf den Ergebnissen von AAR14. S beschreibt Qualität und den Umfang der Datenbasis, Ü den Grad der Übereinstimmung unter Wissenschafterlnnen $[\mathrm{H}=$ hoch, $\mathrm{M}=$ mittel, $\mathrm{N}=$ niedrig] 
Tabelle 5.3 Wichtige Aussagen zur Klimaänderung im Alpenraum und in Österreich basierend auf den Ergebnissen von AAR14. S beschreibł Qualität und den Umfang der Datenbasis, Ü den Grad der Übereinstimmung unter Wissenschafterlnnen [H= hoch, M = mittel, N = niedrig] Table 5.3 Important statements on the climate change for the Alpine region and Austria based on AAR14. S describes Quality and comprehensiveness of the data base, $\ddot{U}$ the degree of agreement among scientists $[H=$ high, $M=$ medium, $N=$ low]

\begin{tabular}{|c|c|c|c|c|}
\hline \multicolumn{2}{|c|}{ Aussage } & \multirow{2}{*}{ S } & \multirow{2}{*}{$\ddot{\mathbf{u}}$} & \multirow{2}{*}{$\begin{array}{l}\text { AAR14 } \\
\text { Kapitel } \\
1 / 3\end{array}$} \\
\hline 1 & $\begin{array}{l}\text { Gegen Ende des letzten Glazials (Würm) erfolgte vor etwa } 30000 \text { Jahren der jüngste eiszeitliche Gletschervor- } \\
\text { stoß bis über den Alpenrand hinaus. Verlässliche Paläoklimadaten dieser Zeit für die Alpen fehlen bislang; man } \\
\text { geht jedoch von einer Absenkung der Jahresmitteltemperatur von mindestens } 10^{\circ} \mathrm{C} \text { im Vergleich zum Holozän } \\
\text { aus, verbunden mit einer ausgeprägten Abnahme des Niederschlags gegen Osten hin. }\end{array}$ & & & \\
\hline 2 & $\begin{array}{l}\text { Die letzten } 2000 \text { Jahre zeigten eine Abfolge von warmen und kalten Perioden, die im Schnitt kühler waren als zu } \\
\text { Beginn und in der Mitte des Holozäns. Den deutlichen, instrumentell belegten Temperaturanstieg des 20. Jahr- } \\
\text { hunderts spiegeln die natürlichen Klima-Archive ebenfalls wider, auch wenn viele Proxy-Datensätze um das Jahr } \\
2000 \text { enden und daher die aktuelle Klimaentwicklung nicht gänzlich erfassen. }\end{array}$ & $M$ & $\mathrm{H}$ & $1 / 3$ \\
\hline 3 & $\begin{array}{l}\text { Die Auflösung und Datierung relativ junger Proxy-Zeitreihen ist für den Alpenraum so gut, dass man direkte } \\
\text { Vergleiche mit verschiedenen klimasteuernden Parametern anstellen kann: So mit der Sonnenaktivität, deren Mi- } \\
\text { nima sich in fast allen Archiven als kältere Perioden widerspiegeln; mit Vulkanausbrüchen, die in Abhängigkeit } \\
\text { von emittiertem Staub und Gasen zu starken Abkühlungen führten; oder mit THGen, die eine wichtige Rolle für } \\
\text { die deutliche Erwärmung seit den 1980er Jahren spielen. Daraus ergibt sich ein Bild verschiedener stevernder } \\
\text { Kräfte, die sich überlagern. }\end{array}$ & $\mathrm{H}$ & $\mathrm{H}$ & $1 / 3$ \\
\hline 4 & $\begin{array}{l}\text { Ein Großteil der nationalen Emissionen (etwa } 79 \% \text { der Gesamtemissionen) erfolgt durch die energetische Nut- } \\
\text { zung von fossiler Energie, als größtem Subsektor ist dem Straßenverkehr mehr als ein Drittel davon zuzuordnen. } \\
\text { Die Zunahme des Holzvorrats im Wald wirkt als Senke im Ausmaß von etwa } 5 \% \text { der Emissionen, ist aber von } \\
\text { Jahr zu Jahr variabel und ist in den letzten Jahren deutlich vermindert. Die Emissionen von Lachgas (vor allem } \\
\text { aufgrund des Einsatzes von Stickstoffdünger im Ackerbau) und Methan (in erster Linie aufgrund von Viehzucht) } \\
\text { entsprechen } 6 \% \text { bzw. } 7 \text { \% der Gesamtemissionen. }\end{array}$ & $\mathrm{H}$ & $\mathrm{H}$ & $1 / 2$ \\
\hline 5 & $\begin{array}{l}\text { Entwicklung der in der THG-Inventur erfassten Emissionen zeigt, dass es sektoral kaum zu Veränderungen } \\
\text { zwischen } 1990 \text { und } 2010 \text { kam. Die für das Gesamtergebnis (Zunahme der Emissionen um } 19 \text { \%) wesentlichen } \\
\text { Ausnahmen sind der Verkehr sowie das weitgehende Wegfallen der Senkenfunktion der Wälder durch Sätti- } \\
\text { gungseffekte der betroffenen Ökosysteme, begleitet von verbesserten forstlichen Möglichkeiten und erhöhtem } \\
\text { Einsatz von Biomasse als Energieträger. }\end{array}$ & $\mathrm{H}$ & $\mathrm{H}$ & $1 / 2$ \\
\hline 6 & $\begin{array}{l}\text { Signifikante Effekte werden auch durch Substanzen bewirkt, die nur eine kurze Aufenthaltsdauer in der } \\
\text { Atmosphäre (wenige Tage) haben. Sie spielen eine wichtige Rolle im Klimasystem - vor allem Aerosole kön- } \\
\text { nen der Erwärmung teilweise entgegenwirken (im Ausmaß etwa der Hälfte des anthropogenen Treibhausef- } \\
\text { fektes) }\end{array}$ & $N$ & M & $1 / 2$ \\
\hline 7 & $\begin{array}{l}\text { Die Temperaturzunahme an hochalpinen Stationen in Österreich ist der in der freien Atmosphäre mit Radio- } \\
\text { sonden in Wien gemessenen sehr ähnlich. In der Stratosphäre ( } 50 \mathrm{hPa}) \text { ist eine deutliche Temperaturabnahme } \\
\text { zu beobachten. Der am Boden im Vergleich mit dem globalen Mittel stärkere Erwärmungstrend im Alpenraum } \\
\text { gleicht sich in höheren Schichten den in mittleren Breiten sonst gefundenen Erwärmungsraten an. }\end{array}$ & $M$ & $M$ & $1 / 3$ \\
\hline 8 & $\begin{array}{l}\text { Die besonders starke negative dekadische Temperaturanomalie von } 1870 \text { bis } 1900 \text { und die besonders starke } \\
\text { positive der aktuellen drei Jahrzehnte führten, verglichen mit dem globalen Mittel, zu einer doppelt so starken } \\
\text { Temperaturanomalie in Österreich und im gesamten Alpengroßraum. }\end{array}$ & $\mathrm{H}$ & $\mathrm{H}$ & $1 / 3$ \\
\hline 9 & $\begin{array}{l}\text { Saisonale Klimaentwicklungen verlaufen nicht notwendigerweise analog zu jener der Jahresmittel. Im Herbst } \\
\text { war die Erwärmung im Alpenraum am geringsten. Phasenweise gab es sogar gegenläufige Entwicklungen, wie } \\
\text { etwa die besonders milden Winter der 1910er Jahre, die durch besonders kühle Sommer konterkariert wurden. } \\
\text { Es war dies eine eher ozeanisch dominierte Phase, die auch besonders feucht und durch vorstoßende Gletscher- } \\
\text { geprägt war. }\end{array}$ & $\mathrm{H}$ & $\mathrm{H}$ & $1 / 3$ \\
\hline 10 & $\begin{array}{l}\text { Die Sonnenscheindauer hat in den letzten } 130 \text { Jahren um ca. } 20 \% \text { zugenommen, im Sommerhalbjahr stärker } \\
\text { als im Winterhalbjahr. Einem ersten ausgeprägten Maximum um } 1950 \text { folgten sonnenscheinärmere Jahrzehnte } \\
\text { bis etwa } 1980 \text { ("global dimming") und eine seither anhaltende "regional brightening" Phase, die die sommerli- } \\
\text { che Sonnenscheindaver in nur drei Jahrzehnten erneut um beinahe } 20 \% \text { ansteigen ließ. }\end{array}$ & $M$ & $M$ & $\begin{array}{l}1 / 3 \\
\text { und } \\
1 / 2\end{array}$ \\
\hline 11 & $\begin{array}{l}\text { Beobachtungen des Luftdrucks in Europa belegen für das südliche Mitteleuropa eine nordwärts Verlagerung } \\
\text { des subtropischen Hochdruckgürtels. Das kann als weiterer Antrieb für die beobachtete deutliche Zunahme der } \\
\text { Sonnenscheindauer herangezogen werden. }\end{array}$ & $M$ & $M$ & $1 / 3$ \\
\hline 12 & $\begin{array}{l}\text { Während die Lufttemperatur während der instrumentellen Messperiode eine sehr einheitliche zeitliche Entwick- } \\
\text { lung im Alpenraum zeigt, ist die Niederschlagsentwicklung im Großraum Alpen regional sehr unterschiedlich } \\
\text { ausgefallen. }\end{array}$ & $\mathrm{H}$ & $\mathrm{H}$ & $1 / 3$ \\
\hline
\end{tabular}




\begin{tabular}{|c|c|c|c|c|}
\hline \multicolumn{2}{|c|}{ Aussage } & \multirow{2}{*}{ S } & \multirow{2}{*}{ Ü } & \multirow{2}{*}{$\begin{array}{l}\text { AAR14 } \\
\text { Kapitel } \\
1 / 3\end{array}$} \\
\hline 13 & $\begin{array}{l}\text { Die Jahressummen des Niederschlags sind in Österreich seit Mitte der 1970er Jahre überall gestiegen, außer } \\
\text { im Südosten, wobei die Zunahme im Norden und inneralpin gleichmäßig ansteigend war (mehr als } 15 \% \text { im } \\
\text { Norden, ca. } 10 \% \text { inneralpin), während der Westen und Südosten stark durch dekadische Minima und Maxima } \\
\text { geprägt war. }\end{array}$ & & & \\
\hline 14 & $\begin{array}{l}\text { Die Winterniederschläge sind in Österreich nördlich des Alpenhauptkammes seit Mitte der 1970er Jahre etwas } \\
\text { gestiegen, südlich des Alpenhauptkammes hingegen deutlich gefallen. In den anderen Jahreszeiten gab es } \\
\text { tendenziell eine Zunahme des Niederschlags in ganz Österreich mit Ausnahme des Südens wo die Trends sehr } \\
\text { gering waren. }\end{array}$ & $\mathrm{H}$ & $\mathrm{H}$ & $1 / 3$ \\
\hline 15 & $\begin{array}{l}\text { Direkte Messungen der Windgeschwindigkeit können derzeit noch nicht für eine statistisch abgesicherte Analyse } \\
\text { von Änderungen der Sturmhäufigkeiten oder -intensitäten herangezogen werden. Aus Luftdruckdaten abgelei- } \\
\text { tete Zeitreihen der Winterstürme zeigen seit den 1870er Jahren starke interannuelle Variabilität, jedoch keine } \\
\text { signifikanten Trends. }\end{array}$ & $\mathrm{H}$ & $\mathrm{H}$ & $1 / 3$ \\
\hline 16 & $\begin{array}{l}\text { Änderungen von Extremwertindizes auf der Basis homogenisierter Zeitreihen täglicher Niederschlagssummen } \\
\text { sind in der anthropogenen Periode meist nicht signifikant. }\end{array}$ & $M$ & $M$ & $1 / 3$ \\
\hline 17 & $\begin{array}{l}\text { Änderungen von Extremwertindizes der täglichen Maximum- und Minimumtemperaturen zeigen in der anthro- } \\
\text { pogenen Periode analoge Trends zum Temperaturmittel, also eine Zunahme der heißen Extremwerte und eine } \\
\text { Abnahme der kalten. Diese Trends sind österreichweit ähnlich und meist signifikant. }\end{array}$ & $\mathrm{H}$ & $\mathrm{H}$ & $1 / 3$ \\
\hline 18 & $\begin{array}{l}\text { Nach Modellberechnungen ist zu erwarten, dass die mittlere Temperatur in Österreich im } 21 \text {. Jahrhundert } \\
\text { weiter ansteigen wird, wobei in den nächsten Jahrzehnten mit etwa }+0,25^{\circ} \mathrm{C} \text { pro Jahrzehnt zu rechnen ist. In } \\
\text { der } 2 \text {. Hälfte des } 21 \text {. Jahrhunderts ist der Temperaturanstieg stark von zukünftigen anthropogenen Emissionen } \\
\text { abhängig. Unter Annahme des A1B Szenarios beschleunigt sich der Temperaturanstieg auf etwa }+0,36^{\circ} \mathrm{C} \text { pro } \\
\text { Jahrzehnt, sodass bis zum Ende des Jahrhunderts mit insgesamt } 3,5^{\circ} \mathrm{C} \text { Erwärmung zu rechnen ist (Vergleichs- } \\
\text { zeitraum } 1961 \text { bis 1990). }\end{array}$ & $\mathrm{H}$ & $\mathrm{H}$ & $1 / 4$ \\
\hline 19 & $\begin{array}{l}\text { Die Niederschlagsänderung im 21. Jahrhundert wird wahrscheinlich eine Zunahme im Winter und eine Abnah- } \\
\text { me im Sommer aufweisen. Dabei wird mit einer erhöhter Niederschlagszunahme nördlich der Alpen im Früh- } \\
\text { ling, Sommer und Herbst gerechnet, während die südlichen und westlichen Teile des Alpenraumes verstärkte } \\
\text { Abnahmen aufweisen. Die Unsicherheiten bezüglich der projizierten Niederschlagszu- und abnahmen sind bis } \\
\text { zur Mitte des } 21 \text {. Jahrhunderts sehr groß, nehmen aber bis zum Ende des Jahrhunderts wieder ab. }\end{array}$ & $M$ & $M$ & $1 / 4$ \\
\hline 20 & Die Häufigkeit im Auftreten von Hitzewellen wird bis zum Ende des Jahrhunderts deutlich ansteigen. & $\mathrm{H}$ & M & $1 / 4$ \\
\hline 21 & $\begin{array}{l}\text { Untersuchungen an Daten der Vergangenheit und konzeptionelle Überlegungen lassen erwarten, dass es auf } \\
\text { Grund der zukünftigen Temperaturerhöhung (und dem damit verbundenen erhöhten Feuchtegehalt der Atmo- } \\
\text { sphäre) zu intensiveren Niederschlagsereignissen insbesondere im Sommer kommen wird. }\end{array}$ & $\mathrm{N}$ & $\mathrm{N}$ & $1 / 4$ \\
\hline
\end{tabular}

\subsection{Literaturverzeichnis 5.8 References}

Allex, B., Liebl, U., Brandenburg, C., Gerersdorfer, T., Czachs, C., 2011. „Hot town, summer in the city“ - Die Auswirkungen von Hitzetagen auf das Freizeit- und Erholungsverhalten sowie das Besichtigungsprogramm von StädtetouristInnen - dargestellt am Beispiel Wiens (Endbericht von Start-Clim2010.F in StartClim2010: Anpassung an den Klimawandel: Weitere Beiträge zur Erstellung einer Anpassungsstrategie für Österreich). Auftraggeber: BMLFUW, BMWF, BMWFJ, ÖBF, Wien.

Armour, K.C., Eisenman, I., Blanchard-Wrigglesworth, E., McCusker, K.E., Bitz, C.M., 2011. The reversibility of sea ice loss in a state-of-the-art climate model. Geophys. Res. Lett. 38, L16705. doi:10.1029/2011GL048739

Auer, I., Böhm, R., Mohnl, H., 1989. Klima von Wien: eine anwendungsorientierte Klimatographie, Beiträge zur Stadtforschung, Stadtentwicklung und Stadtgestaltung. Magistrat der Stadt Wien, Geschäftsgruppe Stadtentwicklung, Stadtplanung und Personal, Geschäftsgruppe Umwelt, Freizeit und Sport, Wien.
Auer, I., Böhm, R., Schöner, W., Hagen, M., 1998. 20th century increase of boundary layer turbidity derived from alpine sunshine and cloudiness series, in: Preprints of the 8th Conference on Mountain Meteorology. American Meteorological Society, Boston, Flagstaff, Arizona, pp. 77-80.

Auer, I., Böhm, R., Korus, E., Schöner, W., 2003. Zeitliche Repräsentativitätsanalyse 50jähriger Klimadatensätze im Hinblick auf die Beschreibung der Variabilität von Extremwerten.

Auer, I., Böhm, R., Jurkovic, A., Lipa, W., Orlik, A., Potzmann, R., Schöner, W., Ungersböck, M., Matulla, C., Briffa, K., Jones, P., Efthymiadis, D., Brunetti, M., Nanni, T., Maugeri, M., Mercalli, L., Mestre, O., Moisselin, J.-M., Begert, M., Müller-Westermeier, G., Kveton, V., Bochnicek, O., Stastny, P., Lapin, M., Szalai, S., Szentimrey, T., Cegnar, T., Dolinar, M., Gajic-Capka, M., Zaninovic, K., Majstorovic, Z., Nieplova, E., 2007. HISTALP—historical instrumental climatological surface time series of the Greater Alpine Region. International Journal of Climatology 27, 17-46. doi:10.1002/joc.1377

Awan, N.K., Formayer, H., 2012. Development of new methods to assess heavy precipitation in the Alps. ACQWA (No. Annual Progress Report). 
Bácsatyai, L., Csaplovics, E., Márkus, I., Sinhuber, A.B., 1997. Digitale Geländemodelle des Neusiedler See-Beckens. Burgenländ. Landesmuseum (Amt d. Bgld. Landesregierung, Abt. XII/1-LM).

Belleflamme, A., Fettweis, X., Erpicum, M., 2011. Evaluation of the present and future general circulation over Greenland simulated by the IPCC AR5/CMIP5 GCMs with the help of a circulation type classification. EGU2011-1206, European Geosciences Union General Assembly 2011.

Böhm, R., 1979. Stadtentwicklung und Trend der Wärmeinselintensität. Archiv für Meteorologie, Geophysik und Bioklimatologie, Serie B 27, 31-46.

Böhm, R., 1998. Urban bias in temperature time series - a case study for the city of Vienna, Austria. Climatic Change 38, 113-128.

Böhm, R., Auer, I., Schöner, W., Hagen, M., 1998. Long alpine barometric time series in different altitudes as a measure for 19th/20th century warming, in: Preprints of the 8th Conference on Mountain Meteorology. American Meteorological Society, Boston, Flagstaff, Arizona, pp. 72-76.

Böhm, R., Auer, I., Schöner, W., 2011. Labor über den Wolken: die Geschichte des Sonnblick-Observatoriums. Böhlau Verlag.

Brundtland, G.H., 1987. Report of the World Commission on environment and development: Our Common Future. United Nations.

Casado, M.J., Pastor, M.A., 2011. How well do IPCC AR4 models simulate circulation types? EGU2011-1206, European Geosciences Union General Assembly 2011.

Craven, G., 2009. What's the Worst That Could Happen?: A Rational Response to the Climate Change Debate, Original edition. ed. Perigee Trade, New York.

Crichton, M., 2004. State of fear: a novel, 1st ed. ed. HarperCollinsPublishers, New York.

Dakos, V., Scheffer, M., Nes, E.H. van, Brovkin, V., Petoukhov, V., Held, H., 2008. Slowing down as an early warning signal for abrupt climate change. PNAS 105, 14308-14312. doi:10.1073/ pnas.0802430105

Daly, H.E., 1990. Sustainable Growth - an Impossible Theorem. Development, Journal of the Society for International Development 45-47.

Damerau, K., 2008. Climate change and energy security-a losing deal? Impacts, Trade-offs and Adaptation Possibilities for Metropolitan Areas. A Scenario Approach for Long-range Energy Planning in the Greater Vienna Region (Diplomarbeit). Universität Wien. Fakultät für Geowissenschaften, Geographie und Astronomie, Wien.

Dobesch, H., Neuwirth, F., 1974. Übersicht über die Ergebnisse aus den hydrometeorologischen Untersuchungen im Gebiet des Neusiedler Sees im Rahmen der Internationalen Hydrologischen Dekade 1966-1974, in: Skoda, F., Lauscher, F. (Eds.), Wetter und Leben, Zeitschrift Für Angewandte Meteorologie. Verlag der Österreichischen Gesellschaft für Meteorologie.

Dobesch, H., Neuwirth, F., 1982. Die Beiträge der Zentralanstalt für Meteorologie und Geodynamik zum Internationalen Hydrologischen Programm 1975-1980, in: Skoda, F., Lauscher (Eds.), Zeitschrift Für Angewandte Meteorologie. Verlag der Österreichischen Gesellschaft für Meteorologie.

Dubois, C., Somot, S., Calmanti, S., Carillo, A., Déqué, M., Dell'Aquilla, A., Elizalde, A., Gualdi, S., Jacob, D., L'Hévéder, B., Li, L., Oddo, P., Sannino, G., Scoccimarro, E., Sevault, F., 2012. Future projections of the surface heat and water budgets of the Mediterranean Sea in an ensemble of coupled atmosphere-oce- an regional climate models. Climate Dynamics 39, 1859-1884. doi:10.1007/s00382-011-1261-4

Eitzinger, J., Kubu, G., Formayer, H., Haas, P., Gerersdorfer, T., Kromp-Kolb, H., 2009. Auswirkungen einer Klimaänderung auf den Wasserhaushalt des Neusiedlersees (Endbericht im Auftrag der Burgenländischen Landesregierung vom 15. Juli 2005) (Boku-Met Report No. 1). Institut für Meteorologie (BOKU-Met) Department Wasser - Atmosphäre - Umwelt Universität für Bodenkultur, Wien.

Emmerich, R., 2004. The Day After Tomorrow.

FAO, 1996. Agro-ecological zoning: Guidelines (No. 73), FAO Soils Bulletin. Soil Resources, Management and Conservation Service. FAO Food and Agriculture Organization of the United Nations, Rome.

Feynman, R.P., 1955. The value of science. Engineering and Science 19, 13-15.

Fischer, E.M., Schär, C., 2010. Consistent geographical patterns of changes in high-impact European heatwaves. Nature Geoscience 3, 398-403. doi:10.1038/ngeo866

Formayer, H., Haas, P., Hofstätter, M., Radanovics, S., Kromp-Kolb, H., 2007. Räumlich und zeitlich hochaufgelöste Temperaturszenarien für Wien und ausgewählte Analysen bezüglich Adaptionsstrategien (Endbericht einer Studie im Auftrag der Wiener Umweltschutzabteilung - MA 22 der Stadt Wien gemeinsam mit der MA 27 - EU-Strategie und Wirtschaftsentwicklung.). Institut für Meteorologie, Universität für Bodenkultur, Wien.

Formayer, H., Matulla, C., Haas, P., Groll, N., 2009. Möglichkeiten statistischer Downscalingverfahren zur Ableitung von Extremereignissen in Österreich aus GCM-Feldern (Endbericht StartClim.5, November 2003) (BOKU-Met Report No. 3).

Fricke, W., Kaminski, U., 2002. Ist die Zunahme von Starkniederschlägen auf veränderte Wetterlagen zurückzuführen?, GAW Brief des Deutschen Wetterdienstes Nr 12.

Früh, B., Becker, P., Deutschländer, T., Hessel, J.-D., Kossmann, M., Mieskes, I., Namyslo, J., Roos, M., Sievers, U., Steigerwald, T., Turau, H., Wienert, U., 2011a. Estimation of Climate-Change Impacts on the Urban Heat Load Using an Urban Climate Model and Regional Climate Projections. Journal of Applied Meteorology and Climatology 50, 167-184. doi:10.1175/2010JAMC2377.1

Früh, B., Roos, M., Koßmann, M., 2011b. Frankfurt am Main im Klimawandel - eine Untersuchung zur städtischen Wärmebelastung, Berichte des Deutschen Wetterdienstes. Selbstverl. des Dt. Wetterdienstes, Offenbach am Main.

GEA, 2012. Global Energy Assessment - Toward a Sustainable Future. Cambridge University Press, Cambridge, UK and New York, NY, USA and the International Institute for Applied Systems Analysis, Laxenburg, Austria.

Godina, R., Lalk, P., Lorenz, P., Müller, G., Weilguni, V., 2006. Hochwasser 2005 - Ereignisdokumentation Teilbericht des Hydrographischen Dienstes. Bundesministerium für Land- und Forstwirtschaft, Umwelt und Wasserwirtschaft Sektion Wasser.

Grazzini, F., Van der Grijn, G., 2002. Central European floods during summer 2002. ECMWF newsletter 96, 18-28.

Habersack, H., Moser, A., 2003. Ereignisdokumentation Hochwasser August 2002, Plattform Hochwasser (No. 1). ZENAR, Universität für Bodenkultur, Bundesministerium für Land und Forstwirtschaft, Umwelt und Wasserwirtschaft, Wien.

Habersack, H., Krapesch, G., 2006. Hochwasser 2005-Ereignisdokumentation der Bundeswasserbauverwaltung, des Forsttechnischen Dienstes für Wildbach-und Lawinenverbauung und des Hydro- 
graphischen Dienstes (Synthesebericht). Bundesministerium für Land-und Forstwirtschaft, Umwelt und Wasserwirtschaft, Wien.

Hansen, J., Sato, M., Kharecha, P., Beerling, D., Berner, R., Masson-Delmotte, V., Pagani, M., Raymo, M., Royer, D.L., Zachos, J.C., 2008. Target atmospheric CO2: Where should humanity aim? The Open Atmospheric Science Journal 2, 217-231. doi: $10.2174 / 1874282300802010217$

Hansen, J., 2009. Storms of My Grandchildren: The Truth About the Coming Climate Catastrophe and Our Last Chance to Save Humanity. Bloomsbury London - Berlin - New York.

Harlfinger, O., Knees, G., 1999. Klimahandbuch der österreichischen Bodenschätzung. Wagner.

Hofstätter, M., Böhm, R., 2011. Machbarkeitsstudie. Vb-artige Wetterlagen als Ursache exzessiver Niederschläge im Alpenraum (Machbarkeitsstudie im Auftrag des Bundesministerium für Land und Forstwirtschaft, Umwelt und Wasserwirtschaft, Sektion VII -Wasser). ZAMG, Wien.

Hofstätter, M., Chimani, B., 2011. WETPAT. Vb-artige Wetterlagen im Klimawandel aus regionalen Klimamodellsimulationen mit Cosmo-CLM (Projektbericht). ZAMG, Wien.

Hofstätter, M., Chimani, B., 2012. Van Bebber's cyclone tracks at $700 \mathrm{hPa}$ in the Eastern Alps for 1961-2002 and their comparison to Circulation Type Classifications. Meteorologische Zeitschrift 21, 459-473. doi:10.1127/0941-2948/2012/0473

Horx, M., 2007. Warum ich nicht an die Klimakatastrophe glaube [WWW Document]. Welt Online. URL http://www.welt.de/politik/article759237/Warum-ich-nicht-an-die-Klimakatastropheglaube.html (accessed 8.31.13).

Hulme, M., 2009. Why we disagree about climate change: understanding controversy, inaction and opportunity. Cambridge University Press, Cambridge, UK; New York.

IPCC, 2013. Climate Change 2013: The Physical Science Basis. Contribution of Working Group I to the Fifth Assessment Report of the Intergovernmental Panel on Climate Change. Cambridge University Press, Cambridge, United Kingdom and New York, NY, USA.

Jacob, D., Göttel, H., Kotlarski, S., Lorenz, P., Sieck, K., 2008. Klimaauswirkungen und Anpassung in Deutschland - Phase 1: Erstellung regionaler Klimaszenarien für Deutschland (Forschungsbericht im Auftrag des Umweltbundesamtes No. 11), Climate Change. Umweltbundesamt, Deutschland, Max-Planck-Institut für Meteorologie (MPI-M), Hamburg.

Jaiser, R., Dethloff, K., Handorf, D., Rinke, A., Cohen, J., 2012. Impact of sea ice cover changes on the Northern Hemisphere atmospheric winter circulation. Tellus A 64, 11595. doi:10.3402/ tellusa.v64i0.11595

James, P., Stohl, A., Spichtinger, N., Eckhardt, S., Forster, C., 2004. Climatological aspects of the extreme European rainfall of August 2002 and a trajectory method for estimating the associated evaporative source regions. Nat. Hazards Earth Syst. Sci. 4, 733-746. doi:10.5194/nhess-4-733-2004

Jonas, H., 1979. Das Prinzip Verantwortung: Versuch einer Ethik für die technologische Zivilisation, 1. Auflage. ed. Insel-Verlag, Frankfurt am Main.

KLIWA Arbeitskreis, 2006. Langzeitverhalten der Starkniederschläge in Baden-Württemberg und Bayern, KLIWA-Projekt A 1.1.3 (No. 8), KLIWA-Berichte. LUBW Landesanstalt für Umwelt, Messungen und Naturschutz, Baden-Württemberg.

Koboltschnig, G.R., Schöner, W., Holzmann, H., Zappa, M., 2009. Glaciermelt of a small basin contributing to runoff under the ex- treme climate conditions in the summer of 2003. Hydrological Processes 23, 1010-1018. doi:10.1002/hyp.7203

Kromp-Kolb, H., Formayer, H., Clementschitsch, L., 2007. Auswirkungen des Klimawandels auf Wien unter besonderer Berücksichtigung von Klimaszenarien (Im Auftrag der Magistratsdirektion der Stadt Wien - Klimaschutzkoordination). Institut für Meteorologie Universität für Bodenkultur.

Kubu, G., 2006. Ökodynamishe Rehabilitierung des Neusiedler See. Dotation Neusiedler See unter Berücksichtigung von Klimaänderungen. (Im Auftrag des Amtes der Burgenländischen Landesregierung Landeswasserbaubezirksamt Schützen am Gebirge).

Kundzewicz, Z.W., Ulbrich, U., Brücher, T., Graczyk, D., Krüger, A., Leckebusch, G.C., Menzel, L., Pińskwar, I., Radziejewski, M., Szwed, M., 2005. Summer Floods in Central Europe - Climate Change Track? Natural Hazards 36, 165-189. doi:10.1007/ s11069-004-4547-6

Kyselý, J., Kalvová, J., Květoň, V., 2000. Heat Waves in the South Moravian Region during the Period 1961-1995. Studia Geophysica et Geodaetica 44, 57-72. doi:10.1023/A:1022009924435

Latif, M., Keenlyside, N.S., 2009. El Niño/Southern Oscillation response to global warming. PNAS 106, 20578-20583. doi:10.1073/pnas.0710860105

Lautenschlager, M., Keuler, K., Wunram, C., Keup-Thiel, E., Schubert, M., Will, A., Rockel, B., Boehm, U., 2009. Climate Simulation with CLM, Scenario A1B run no.2, Data Stream 3: European region MPI-M/MaD. WDCC at DKRZ. doi:10.1594/WDCC/ CLM_A1B_2_D3

Lautenschlager, M., Keuler, K., Wunram, C., Keup-Thiel, E., Schubert, M., Will, A., Rockel, B., Boehm, U., 2011. Climate Simulation with CLM, Climate of the 20th Century run no.3, Data Stream 3: European region MPI-M/MaD. WDCC at DKRZ. doi:10.1594/WDCC/CLM_C20_3_D3

Lenton, T.M., 2012. Arctic Climate Tipping Points. AMBIO 41, 10-22. doi:10.1007/s13280-011-0221-x

Lenton, T.M., Held, H., Kriegler, E., Hall, J.W., Lucht, W., Rahmstorf, S., Schellnhuber, H.J., 2008. Inaugural Article: Tipping elements in the Earth's climate system. Proceedings of the $\mathrm{Na}-$ tional Academy of Sciences 105, 1786-1793. doi:10.1073/ pnas. 0705414105

Leuschner, A., 2012. Die Glaubwürdigkeit der Wissenschaft: eine wissenschafts- und erkenntnistheoretische Analyse am Beispiel der Klimaforschung, Science studies. Transcript, Bielefeld.

Liu, J., Curry, J.A., Wang, H., Song, M., Horton, R.M., 2012. Impact of declining Arctic sea ice on winter snowfall. PNAS. doi:10.1073/pnas.1114910109

Loibl, W., Tötzer, T., Köstl, M., Züger, H., Knoflacher, M., 2011. Modelling Micro-climate Characteristics for Urban Planning and Building Design, in: Hřebíček, J., Schimak, G., Denzer, R. (Eds.), Presented at the 9th IFIP WG 5.11 International Symposium on Environmental Software Systems: Frameworks of eEnvironment, ISESS 2011; Brno; Czech Republic; 27 June 2011 through 29 June 2011, Springer Berlin Heidelberg, Berlin, Heidelberg, pp. 605-617. doi:10.1007/978-3-642-22285-6_65

Malitz, G., Schmidt, T., 1997. Hydrometeorologische Aspekte des Sommerhochwassers der Oder 1997, in: Deutscher Wetterdienst (Ed.), Klimastatusbericht 1997. Offenbach, Frankfurt, pp. 28-36.

Meinharter, E., Balas, M., 2011. Anpassungsempfehlungen für urbane Grün- und Freiräume in österreichischen Städten und Stadtregionen (Endbericht von StartClim2010. B in StartClim2010: Anpassung an den Klimawandel: Weitere Beiträge zur Erstellung 
einer Anpassungsstrategie für Österreich). Auftraggeber: BMLFUW, BMWF, BMWFJ, ÖBF.

Mesinger, F., Strickler, R.F., 1982. Effect of mountains on Genoa cyclogenesis. Journal of the Meteorological Society of Japan -Ser. II 60, 326-338.

Moshammer, H., Hutter, H.P., Frank, A., Gerersdorfer, T., Hlava, A., Sprinzl, G., Leitner, B., 2006. Einflüsse der Temperatur auf Mortalität und Morbidität in Wien (StartClim2005.A1a Teilprojekt von StartClim2005 - Klimawandel und Gesundheit!). Universität für Bodenkultur, Department für Wasser - Atmosphäre - Umwelt Institut für Meteorologie, Wien.

Moshammer, H., Hutter, H.P., Gerersdorfer, T., 2009. Einfluss von Adaptationsmaßnahmen auf das akute Sterberisiko in Wien durch Temperaturextreme (StartClim2008.A Teilprojekt von StartC$\lim 2008)$. Institut für Umwelthygiene Medizinische Universität Wien, ZPH, Universität für Bodenkultur,Institut für Meteorologie, Wien, Austria.

Mudelsee, M., Börngen, M., Tetzlaff, G., Grünewald, U., 2004. Extreme floods in central Europe over the past 500 years: Role of cyclone pathway „Zugstrasse Vb“. Journal of Geophysical Research 109. doi:10.1029/2004JD005034

Mursch-Radlgruber, E., Trimmel, H., Gerersdorfer, T., 2009. Räumliche Differenzierung der mikroklimatischen Eigenschaften von Wiener Stadtstrukturen und Anpassungsmaßnahmen. Ergebnisse kleinklimatischer Messungen. Teil 2 der Studie „Räumlich und zeitlich hoch aufgelöste Temperaturszenarien für Wien und ausgewählte Analysen bezüglich Adaptationsstrategien“. Wiener Umweltschutzabteilung (MA 22), EU-Strategie und Wirtschaftsentwicklung (MA 27).

Muthers, S., Matzarakis, A., Koch, E., 2010. Climate change and mortality in Vienna-A human biometeorological analysis based on regional climate modeling. International Journal of Environmental Research and Public Health 7, 2965-2977. doi:10.3390/ ijerph7072965

Nakicenovic, N., 2003. Global energy perspectives and the role of technology. Elektrotech. Inftech. 120, 377-381. doi:10.1007/ BF03054921

National Research Council, 2013. Abrupt Impacts of Climate Change: Anticipating Surprises. The National Academies Press, Washington, DC.

Oke, T.R., 1982. The energetic basis of the urban heat island. Quarterly Journal of the Royal Meteorological Society 108, 1-24. doi:10.1002/qj.49710845502

Oke, T.R., 1987. Boundary Layer Climates. Routledge.

ÖNORM, 1983. Vereinfachte Berechnung des zeitbezogenen Wärmeverlustes (Heizlast) von Gebäuden.

Oreskes, N., Conway, E.M.M., 2010. Merchants of doubt: how a handful of scientists obscured the truth on issues from tobacco smoke to global warming, 1st U.S. ed. ed. Bloomsbury Press, New York.

ÖSTA, n.d. Erste Landesaufnahme. 1773-1781. Kriegs-Charte des Erzherzogtums Oesterreich unter der Enns, 1:28.800 (Wien). Kolorierte Handzeichnung, 122 Sectionen à $71,5 \times 47 \mathrm{~cm}$, Sectionen 59,60,61,70,71,72,81,82,83: Theil deren Vierteln unter Wiener Wald, und unter Manhartsberg, Österreichisches Staatsarchiv/ Kriegsarchiv: B IX a 242

Perovich, D.K., Richter-Menge, J.A., Jones, K.F., Light, B., 2008. Sunlight, water, and ice: Extreme Arctic sea ice melt during the summer of 2007. Geophys. Res. Lett. 35, L11501. doi:10.1029/2008GL034007
Petoukhov, V., Semenov, V.A., 2010. A link between reduced BarentsKara sea ice and cold winter extremes over northern continents. J. Geophys. Res. 115, D21111. doi:10.1029/2009JD013568

Philipp, A., Bartholy, J., Beck, C., Erpicum, M., Esteban, P., Fettweis, X., Huth, R., James, P., Jourdain, S., Kreienkamp, F., Krennert, T., Lykoudis, S., Michalides, S.C., Pianko-Kluczynska, K., Post, P., Álvarez, D.R., Schiemann, R., Spekat, A., Tymvios, F.S., 2010. Cost733cat - A database of weather and circulation type classifications. Physics and Chemistry of the Earth, Parts A/B/C, Classifications of Atmospheric Circulation Patterns - Theory and Applications 35, 360-373. doi:10.1016/j.pce.2009.12.010

Philipp, A., Della-Marta, P.M., Jacobeit, J., Fereday, D.R., Jones, P.D., Moberg, A., Wanner, H., 2007. Long-Term Variability of Daily North Atlantic-European Pressure Patterns since 1850 Classified by Simulated Annealing Clustering. J. Climate 20, 4065-4095. doi:10.1175/JCLI4175.1

Pichler, H., Steinacker, R., 1987. On the synoptics and dynamics of orographically induced cyclones in the Mediterranean. Meteorology and Atmospheric Physics 36, 108-117. doi:10.1007/ BF01045144

PIK, 2012. Turn down the heat: Why a $4^{\circ}$ warmer world must be avoided (A Report for the World Bank by the Potsdam Institute for Climate Impact Research and Climate Analytics), World Bank Publications. International Bank for Reconstruction and Development / The World Bank.

Piribauer, S., Strasser, S., 2010. „Hot in the city“ - Klimadatenreihenanalyse in Hinblick auf die Vulnerabilität des Wiener Städtetourismus. Bakkalaureatsarbeit zur Erlangung des akademischen Grades eines(r) Bakkalaureus/Bakkalaurea der Technischen Wissenschaften der Studienrichtung Kulturtechnik und Wasserwirtschaft an der Universität für Bodenkultur,Wien.

Planet under Pressure, 2012. State of the planet declaration. New knowledge towards solutions. London. http://www.planetunderpressure2012.net/pdf/state_of_planet_declaration.pdf.

Popper, K., 1955. Logik der Forschung, [Springer, Wien 1935], 11. Aufl. 2005. Ed. Mohr Siebeck, Tübingen.

Prettenthaler, F., Gobiet, A. (Eds.), 2008. Heizen und Kühlen im Klimawandel. Erste Ergebnisse zu den künftigen Änderungen des Energiebedarfs für die Gebäudetemperierung Studien zum Klimawandel in Österreich. Verlag der Österreichischen Akademie der Wissenschaften, Wien.

Pröbstl, U., Jiricka, A., Schauppenlehner, T., 2007. See-Vision: Einfluss von klimawandelbedingten Wasserschwankungen im Neusiedler See auf die Wahrnehmung und das Verhalten von Besucherinnen und Besuchern (Endbericht StartClim2006.D3 Teilprojekt von StartClim2006). Finanziert aus Mitteln des BMLFUW, des BMGFJ, des BMWF, des BMWA und der Österreichischen Hagelversicherung, Wien.

Řezáčová, D., Kašpar, M., Müller, M., Sokol, Z., Kakos, V., Hanslian, D., Pešice, P., 2005. A comparison of the flood precipitation episode in August 2002 with historic extreme precipitation events on the Czech territory. Atmospheric Research 77, 354-366. doi:10.1016/j.atmosres.2004.10.008

Ruckstuhl, C., Philipona, R., Behrens, K., Collaud Coen, M., Dürr, B., Heimo, A., Mätzler, C., Nyeki, S., Ohmura, A., Vuilleumier, L., Weller, M., Wehrli, C., Zelenka, A., 2008. Aerosol and cloud effects on solar brightening and the recent rapid warming. Geophys. Res. Lett. 35, L12708. doi:10.1029/2008GL034228

Rudari, R., Entekhabi, D., Roth, G., 2004. Terrain and MultipleScale Interactions as Factors in Generating Extreme Preci- 
pitation Events. Journal of Hydrometeorology 5, 390-404. doi:10.1175/1525-7541(2004)005<0390:TAMIAF>2.0.CO;2

Rudolf, B., Rapp, J., 2003. Das Jahrhunderthochwasser der Elbe: Synoptische Wetterentwicklung und klimatologische Aspekte, in: Deutscher Wetterdienst (Ed.), Klimastatusbericht 2002. Offenbach, pp. 172-187.

Sailor, D.J., 2001. Relating residential and commercial sector electricity loads to climate-evaluating state level sensitivities and vulnerabilities. Energy 26, 645-657. doi:10.1016/S03605442(01)00023-8

Schär, C., Vidale, P.L., Lüthi, D., Frei, C., Häberli, C., Liniger, M.A., Appenzeller, C., 2004. The role of increasing temperature variability in European summer heatwaves. Nature 427, 332-336. doi:10.1038/nature02300

Scheffer, M., Bascompte, J., Brock, W.A., Brovkin, V., Carpenter, S.R., Dakos, V., Held, H., van Nes, E.H., Rietkerk, M., Sugihara, G., 2009. Early-warning signals for critical transitions. Nature 461, 53-59. doi:10.1038/nature08227

Schellnhuber, H.J., 2009. Tipping elements in the Earth System. Proceedings of the National Academy of Sciences 106, 2056120563. doi:10.1073/pnas.0911106106

Schicker, I., Radanovics, S., Seibert, P., 2010. Origin and transport of mediterranean moisture and air. Atmospheric Chemistry and Physics 10, 5089-5105. doi:10.5194/acp-10-5089-2010

Schöner, W., Böhm, R., 2007. A statistical mass-balance model for reconstruction of LIA ice mass for glaciers in the European Alps. Annals of Glaciology 46, 161-169. doi: $10.3189 / 172756407782871639$

Schöner, W., Böhm, R., Auer, I., 2012. 125 years of high-mountain research at Sonnblick-Observatory (Austrian Alps) - from „the house above the clouds" to a unique research platform. Theoretical and Applied Climatology 110, 491-498. doi:10.1007/s00704012-0689-8

Schönerklee, M., Kinner, P., Heiß, G., Soja, G., Kleissner, A., Schindler, J., Treitler, R., 2008. Cli-mate Change - Impacts on hydrology and regional development. Scientific Study on the Impacts of the Decreasing Water Level of Lake Neusiedl, Austria. Vortrag beim IWA World Water Congress, Wien - Kongresszentrum; 09.09.2008-13.09.2008.

Schörghuber, S., Rammer, W., Lexer, M.J., 2012. Analyse von Vulnerabilität und Anpassungsmaßnahmen an den Klimawandel im Biosphärenpark Wienerwald (Endbericht vonStartClim2009.C in StartClim2009: Anpassung an den Klimawandel: Beiträge zur Erstellung einer Anpassungsstrategie für Österreich, Auftraggeber: BMLFUW, BMWF, BMWFJ, ÖBF). Universität für Bodenkultur, Department für Wasser - Atmosphäre - Umwelt, Wien, Austria.

Screen, J.A., Simmonds, I., 2012. Declining summer snowfall in the Arctic: causes, impacts and feedbacks. Clim Dyn 38, 2243-2256. doi:10.1007/s00382-011-1105-2

Seibert, P., Frank, A., Formayer, H., 2007. Synoptic and regional patterns of heavy precipitation in Austria. Theor. Appl. Climatol. 87, 139-153. doi:10.1007/s00704-006-0198-8

Sevault, F., Somot, S., Beuvier, J., 2009. A regional version of the NEMO ocean engine on the Mediterranean Sea: NEMOMED8 user's guide. Note de travail du Groupe de Météorologie Grande Echelle et Climat.

Sievers, U., 1990. Dreidimensionale Simulation in Stadtgebieten. Schriftenreihe Umweltmeteorologie 15, 36-43.

Sievers, U., 1995. Verallgemeinerung der Stromfunktionsmethode auf drei Dimensionen. Meteorologische Zeitschrift 4, 3-15.
Soja, G., Züger, J., Knoflacher, M., Kinner, P., Soja, A.-M., 2013. Climate impacts on water balance of a shallow steppe lake in Eastern Austria (Lake Neusiedl). Journal of Hydrology 480, 115-124. doi:10.1016/j.jhydrol.2012.12.013

Solomon, S., Plattner, G.-K., Knutti, R., Friedlingstein, P., 2009. Irreversible climate change due to carbon dioxide emissions. PNAS pnas.0812721106. doi:10.1073/pnas.0812721106

Spämann, R., 1979. Technische Eingriffe in die Natur als Problem der politischen Ethik. Scheidewege 9/4, 476-497.

Statistik Austria, 2012. Demographisches Jahrbuch 2011. (Seite 60)

Steinnocher, K., 1996. Integration of spectral and spatial classification methods for building a land-use model of Austria, in: Kraus, K., Waldhäusl, P. (Eds.), XVIIIth ISPRS Congress Technical Commission IV: Mapping and Geographic Information Systems. Presented at the XVIIIth ISPRS Congress Technical Commission IV, International Society for Photogrammetry and Remote Sensing, Vienna, Austria, pp. 841-846.

Stroeve, J., Holland, M.M., Meier, W., Scambos, T., Serreze, M., 2007. Arctic sea ice decline: Faster than forecast. Geophys. Res. Lett. 34, L09501. doi:10.1029/2007GL029703

Thaler, S., Holawe, F., Mursch-Radlgruber, E., 2005. Fluctuations in heat related mortality in Vienna, in: Deutscher Wetterdienst (Offenbach, M. (Ed.), Annalen der Meteorologie Nr. 41, Band 1 und 2: 17th International Congress of Biometeorology ICB 2005. Presented at the 17th International Congress of Biometeorology ICB 2005, Deutscher Wetterdienst, Offenbach am Main.

Thaler, S., Holawe, F., Mursch-Radlgruber, E., 2008. Are there any influences of meteorological conditions on mortality fluctuations in Vienna, Austria, in: American Meteorological Society (Ed.), Presented at the 18th Conference on Atmospheric BioGeosciences / 28th Conference on Agricultural and Forest Meteorology, Orlando.

Tietsche, S., Notz, D., Jungclaus, J.H., Marotzke, J., 2011. Recovery mechanisms of Arctic summer sea ice. Geophys. Res. Lett. 38, L02707. doi:10.1029/2010GL045698

Ulbrich, U., Brücher, T., Fink, A.H., Leckebusch, G.C., Krüger, A., Pinto, J.G., 2003. The central European floods of August 2002: Part 2 -Synoptic causes and considerations with respect to climatic change. Weather 58, 434-442. doi:10.1256/wea.61.03B

UNCED, 1992. Rio - Erklärung über Umwelt und Entwicklung, in: Report of the United Nations Conference on Environment and Development, Rio de Janeiro.

UNFCCC, 1992. Rahmenübereinkommen der Vereinten Nationen über Klimaänderungen.

Van Bebber, W.J., 1882. Typische Witterungserscheinungen. Archiv der Deutschen Seewarte Bd. V 1882.

Van Bebber, W.J., 1891. Die Zugstrassen der barometrischen Minima nach den Bahnenkarten der Deutschen Seewarte für den Zeitraum 1875-1890. 361-366.

Van Bebber, W.J., Köppen, W., 1895. Die Isobarentypen des Nordatlantischen Ozeans und Westeuropas, ihre Beziehung zur Lage und Bewegung der Barometrischer Maxima und Minima. Archiv deutsch. Seewarte 18, 1-27.

Van Ulden, A.P., van Oldenborgh, G.J., 2006. Large-scale atmospheric circulation biases and changes in global climate model simulations and their importance for climate change in Central Europe. Atmospheric Chemistry and Physics 6, 863-881.

Wadhams, P., 2012. Arctic Ice Cover, Ice Thickness and Tipping Points. AMBIO 41, 23-33. doi:10.1007/s13280-011-0222-9

Wanner, H., Rickli, R., Salvisberg, E., Schmutz, C., Schüepp, M., 1997. Global climate change and variability and its influence on 
Alpine climate - concepts and observations. Theor Appl Climatol 58, 221-243. doi:10.1007/BF00865022

Wild, M., Gilgen, H., Roesch, A., Atsumu, O., Long, C.N., Dutton, E.G., Forgan, B., Kallis, A., Russak, V., Tsvetkov, A., 2005. From Dimming to Brightening: Decadal Changes in Solar Radiation at Earth's Surface. Science 308, 847-850. doi:10.1126/ science. 1103215

ZAMG, 2012. Gewitterserie ab Mitte Juni 2012 in der Steiermark. ZAMG, Universität für Bodenkultur, Wien.

Zängl, G., 2004. Numerical simulations of the 12-13 August 2002 flooding event in eastern Germany. Quarterly Journal of the Royal Meteorological Society 130, 1921-1940. doi:10.1256/qj.03.152

Žuvela -Aloise, M., 2011. SISSI: Simulationen von Städtischen Klimaszenarien (Projektbericht).
Žuvela -Aloise, M., Nemec, J., Früh, B., 2012. Dynamical modelling of urban climate of Vienna. Presented at the 8th International conference on Urban Climate and 10th Symposium on the Urban Environment (ICUC8), Dublin, Ireland.

Žuvela-Aloise, M. 2013. FOCUS-I: Adaption and mitigation of the climate change impact on urban heat stress based on model runs derived with an urban climate model, ACRP final report, pp. 67.

Žuvela-Aloise, M., Koch R., Neureiter, A., Böhm R. And S. Buchholz (2014). Reconstructing urban climate of Vienna based on historical maps dating to the early instrumental period, Urban Climate, DOI: 10.1016/j.uclim.2014.04.002 



\section{Band 2: Klimawandel in Österreich: Auswirkungen auf Um- welt und Gesellschaft}

\section{Volume 2: Climate Change in Austria: Environmental and Societal Implications}

Kapitel 1: Zur Kopplung zwischen Treiber- und Reaktionssystemen sowie zur Bewertung von Folgen des Klimawandels Kapitel 2: Der Einfluss des Klimawandels auf die Hydrosphäre

Kapitel 3: Der Einfluss des Klimawandels auf die Biosphäre und Ökosystemleistungen

Kapitel 4: Der Einfluss des Klimawandels auf die Reliefsphäre

Kapitel 5: Der Einfluss des Klimawandels auf die Pedosphäre

Kapitel 6: Der Einfluss des Klimawandels auf die Anthroposphäre 
